\title{
Republic of Armenia: 2010 Article IV Consultation, First Review Under the Extended Financing Facility and Extended Credit Facility, and Request for Modification of Performance Criteria-Staff Report; Public Information Notice and Press Release on the Executive Board Discussion.
}

Under Article IV of the IMF's Articles of Agreement, the IMF holds bilateral discussions with members, usually every year. In the context of a combined discussion of the 2010 Article IV consultation with Armenia and first review under the extended financing facility and extended credit facility, and request for modification of performance criteria, the following documents have been released and are included in this package:

- $\quad$ The staff report for the combined 2010 Article IV consultation and first review under the extended financing facility and extended credit facility, and request for modification of performance criteria, prepared by a staff team of the IMF, following discussions that ended on September 24, 2010, with the officials of the Republic of Armenia on economic developments and policies. Based on information available at the time of these discussions, the staff report was completed on November 12, 2010. The views expressed in the staff report are those of the staff team and do not necessarily reflect the views of the Executive Board of the IMF.

- $\quad$ A Public Information Notice (PIN) and Press Release, summarizing the views of the Executive Board as expressed during its December 1, 2010, discussion of the staff report on issues related to the Article IV consultation and the IMF arrangement, respectively.

The documents listed below have been or will be separately released.

Letter of Intent sent to the IMF by the authorities of the Republic of Armenia*

Technical Memorandum of Understanding*

*Also included in Staff Report

The policy of publication of staff reports and other documents allows for the deletion of market-sensitive information.

Copies of this report are available to the public from

International Monetary Fund • Publication Services

$70019^{\text {th }}$ Street, N.W. • Washington, D.C. 20431

Telephone: (202) 623-7430 • Telefax: (202) 623-7201

E-mail: publications@imf.org Internet: http://www.imf.org

\section{International Monetary Fund Washington, D.C.}




\title{
INTERNATIONAL MONETARY FUND
}

\section{REPUBLIC OF ARMENIA}

\section{Staff Report for the 2010 Article IV Consultation Discussions, First Review Under the Extended Financing Facility and Extended Credit Facility, and Request for Modification of Performance Criteria}

\author{
Prepared by the Middle East and Central Asia Department \\ Approved by Ratna Sahay (MCD) and Thomas Dorsey (SPR)
}

November 12, 2010

\section{EXECUTIVE SUMMARY}

Background: The global crisis brought an end to a long period of high, but unsustainable growth. The sharp contraction in exports, remittances, and FDI, and the postponement of an exchange rate devaluation led to a crisis of confidence in Armenia and an enormous drop in output. A strong policy response from the authorities, supported by the international community, including the IMF, helped to soften the impact of the crisis and recover confidence, albeit at the cost of a substantial rise in public debt. Economic activity is now picking up, but the pace of recovery remains slow.

Policy challenges: Armenia's principal policy challenges center on the need to maintain fiscal and debt sustainability, adjust to the uncertain external environment, and achieve sustainable medium-term growth and poverty reduction.

\section{Key recommendations:}

- Implement a fiscal exit strategy to address medium-term fiscal and debt vulnerabilities. To this end, the deficit should be reduced to below 4 percent in 2011, and to about $2 \frac{1}{2}$ percent of GDP by 2013. This would put debt back on a downward trend reaching 36 percent of GDP by 2015 .

- Continue to target a pre-announced inflation rate, preventing temporary price increases from becoming entrenched, and enhance tools for making monetary policy more effective.

- Adhere to a flexible exchange rate regime, intervening in the foreign exchange market only to smooth market volatility and safeguard foreign exchange reserves.

- Continue implementing prudential measures to preserve financial stability, maintain banking sector soundness, and deepen financial markets.

- Continue structural reforms to enhance external competitiveness and strengthen competition in the economy. Key areas include tax administration and tax policy reform, strengthening the business environment, and addressing corruption.

- Expand well-targeted social assistance programs to reduce poverty. 


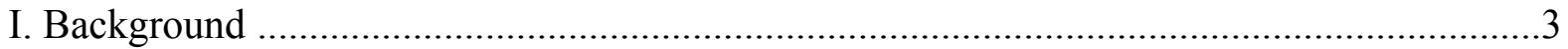

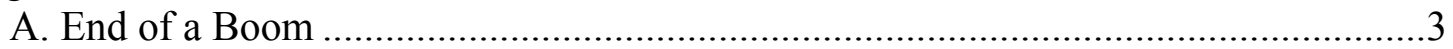

B. A Fragile Recovery is Proceeding ...................................................................4

II. Facing a Sobering Outlook: Medium-Term Policy Challenges ....................................6

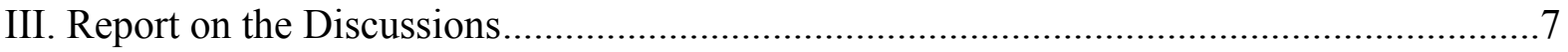

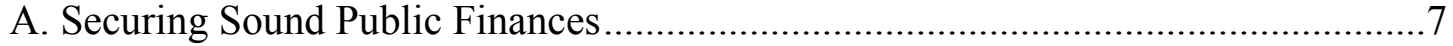

B. Adjusting to the External Environment............................................................ 9

C. Creating a More Dynamic Economy..................................................................... 11

IV. The EFF/ECF-Supported Program Remains on Track ............................................13

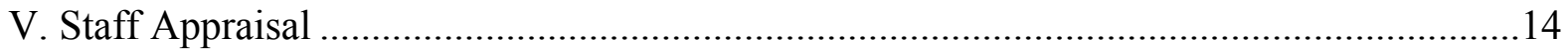

Tables

1. Selected Economic and Financial Indicators, 2006-13 …........................................... 17

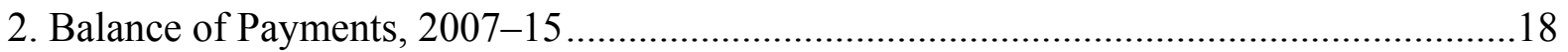

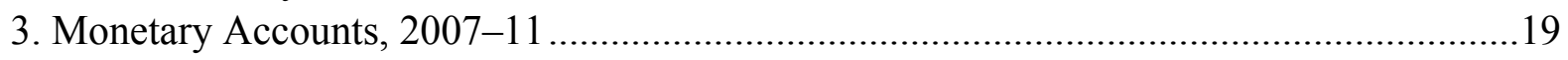

4. Financial Soundness Indicators for the Banking Sector, 2005-10 ...............................20

5. Central Government Operations (in billions of drams), 2008-13 ....................................21

6. Central Government Operations (in percent of GDP), 2008-13 …...............................22

7. Medium-Term Macroeconomic Framework, 2007-15.................................................23

8. Proposed Fund Disbursements and Timing of Reviews Under a Three-Year EFF/ECF

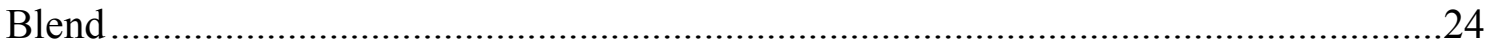

9. Indicators of Capacity to Repay the Fund, 2010 18 .................................................25

10. Structural Benchmarks for Future Implementation Under the EFF/ECF ......................26

11. Millennium Development Goals, 1993-2010 .......................................................27

Box

1. Revisions of National Accounts Estimates ..............................................................4

Appendixes

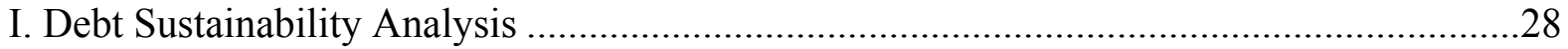

II. Assessment of the Real Exchange Rate and Competitiveness ........................................41

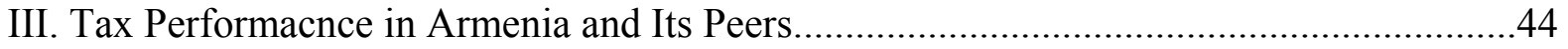

IV. Business Climate and Competitiveness ................................................................48

Attachments

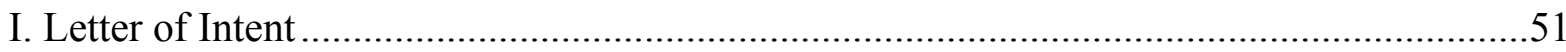

II. Technical Memorandum of Understanding............................................................61 


\section{BACKGROUND ${ }^{1}$}

\section{A. End of a Boom}

\section{Armenia experienced one of the} highest growth rates in the world prior to the global crisis. Real GDP growth averaged 12 percent per year during 2000 07 , leading to a sharp increase in per capita income and a fall in poverty. This growth depended on remittances, however, which were funneled, in particular, to construction. The share of construction in real GDP peaked at 26 percent in $2008 .^{2}$

\section{The global crisis brought an end}

to this boom. The sharp contraction in exports, remittances, and FDI, and the postponement of an exchange rate devaluation led to a crisis of confidence in Armenia and an enormous drop in output.

GDP and Remittances Growth, and Contribution of Construction Sector

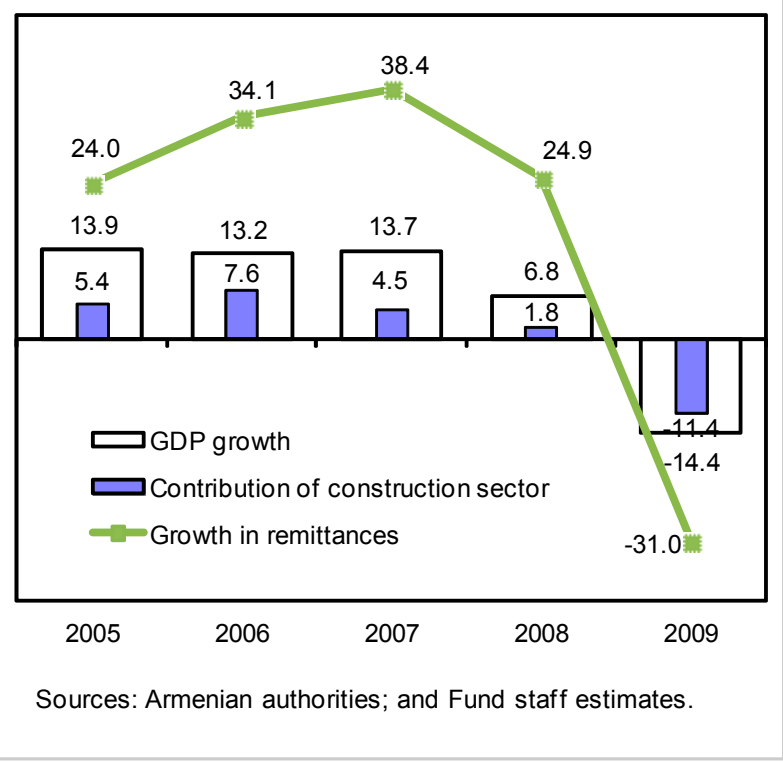

A strong policy response from the authorities, paired with substantial support from the international community, including the IMF, helped to soften the impact of the crisis and recover confidence, albeit at the cost of a substantial rise in public debt. The crisis underscored that a growth strategy predicated on private remittance inflows into nontradables is not sustainable and exposes the country's vulnerabilities to external shocks.

3. At the time of the last Article IV discussions in November 2008, the IMF Board encouraged the authorities to pursue prudent monetary and fiscal policies, enhance their inflation targeting framework, strengthen the financial system, and implement far-reaching structural reforms. The authorities' policy response was broadly in line with Fund advice. While fiscal and monetary policies shifted to a countercyclical mode during the crisis, all major reforms are on-track except for some delays in tax policy and business environment reforms.

\footnotetext{
${ }^{1}$ This report is based on discussions held in Yerevan during September 9-24, 2010. The staff team comprised R. Sahay (Head), V. Bacalu, A. Bordon, A. Weber (all MCD), A. El-Ganainy (FAD), E. Gemayel (SPR), D. Saeju (MCM), and G. Tolosa (Resident Representative). A. Manookian and A. Ghazaryan of the resident representative office, and G. Sargsyan (OED) also participated in the meetings. The mission met with the Prime Minister, the Governor of the Central Bank, the Minister of Finance, and the Minister of Economy, as well as with private and financial sector representatives, NGOs, and parliamentarians.

${ }^{2}$ The authorities' efforts to improve the national accounts data are outlined in Box 1.
} 


\section{Box 1. Armenia: Revisions of National Accounts Estimates}

The National Statistical Service (NSS) of Armenia is undertaking a revision of the annual and quarterly GDP data series since 2008. The annual estimates of GDP at constant prices will be revised to include improved statistical techniques for deriving volume measures of GDP. The quarterly NSS GDP data at current and at constant prices will also be revised, in accordance with the System of National Accounts. Quarterly GDP estimates at current prices will be compiled from discrete data sources only, since the NSS has successfully redesigned its data collection program to collect source data on a discrete basis rather than a cumulative one. The quarterly GDP estimates at constant prices will be compiled as chain volume measures. The NSS plans to disseminate these revised annual and quarterly GDP estimates for 2008-Q1-2011 in June 2011.

As of January 2011, the NSS will discontinue monthly GDP compilation, and will start compiling a monthly indicator of economic activity (IEA), following international best practices. The monthly IEA is not a measure of value added (GDP), but an aggregated measure of production. The IEA will be disseminated on an aggregated level only - for the total economy. The presentation will include monthto-month growth rates and month-to-corresponding month of the previous year growth rates. Time series of chain-linked IEA will also be published. The index will be reconstructed back to January 2000 .

4. The political situation remains broadly unchanged, and hoped-for improvements in relations with neighbors have not materialized. Steps toward the opening of the land border with Turkey have not progressed, and tensions remain with Azerbaijan on the Nagorno-Karabakh dispute. Armenia's closed borders with Turkey and Azerbaijan pose a heavy economic cost, as it is a land-locked country. A reopening of the land border with Turkey would greatly benefit the Armenian economy (Box3, Country Report No. 09/316).

\section{B. A Fragile Recovery is Proceeding}

5. Economic activity is recovering from the sharp downturn last year, but the pace of the recovery remains slow. Real GDP growth was 3.1 percent through the first eight months of the year, driven by a recovery in services and industry. Compared to the $6 \frac{1}{2}$ percent rate observed in the first half of 2010, the pace of growth has slowed, reflecting unfavorable weather conditions and an unprecedented decline in agricultural output. The fiscal stimulus - financed by a

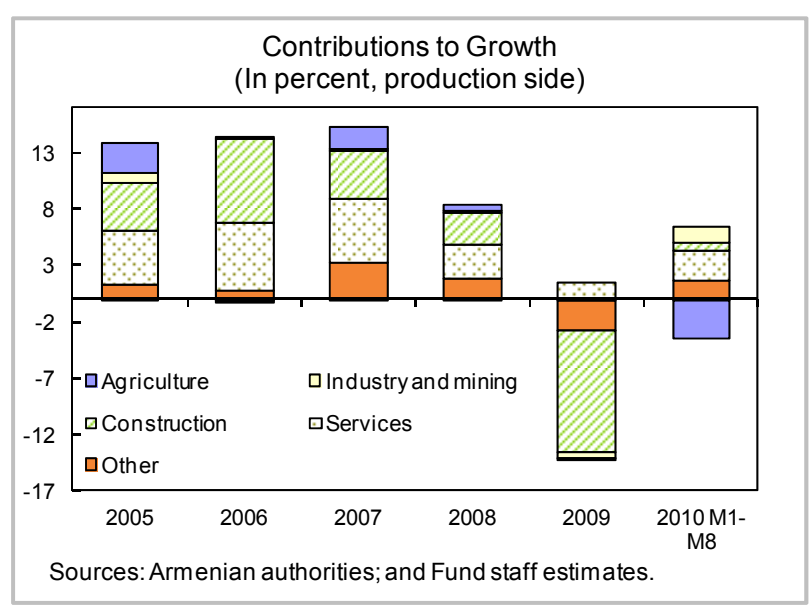


sharp increase in public borrowing - is being unwound. On this basis and the expectation of a modest recovery of the agricultural sector in the coming months, growth for the year is now projected to be 4 percent.

\section{Domestic and external supply} shocks have pushed up inflation. After falling to $5 \frac{1}{2}$ percent earlier in the year, sharp increases in food prices, including fruits, vegetables, and bread, led to a spike in headline inflation to 8.6 percent year-on-year in September. With only a modest recovery in agriculture expected in the coming months, end-year inflation has been revised upward from 6.2 to 7.1 percent. Food prices are expected to decline next year, and with appropriate

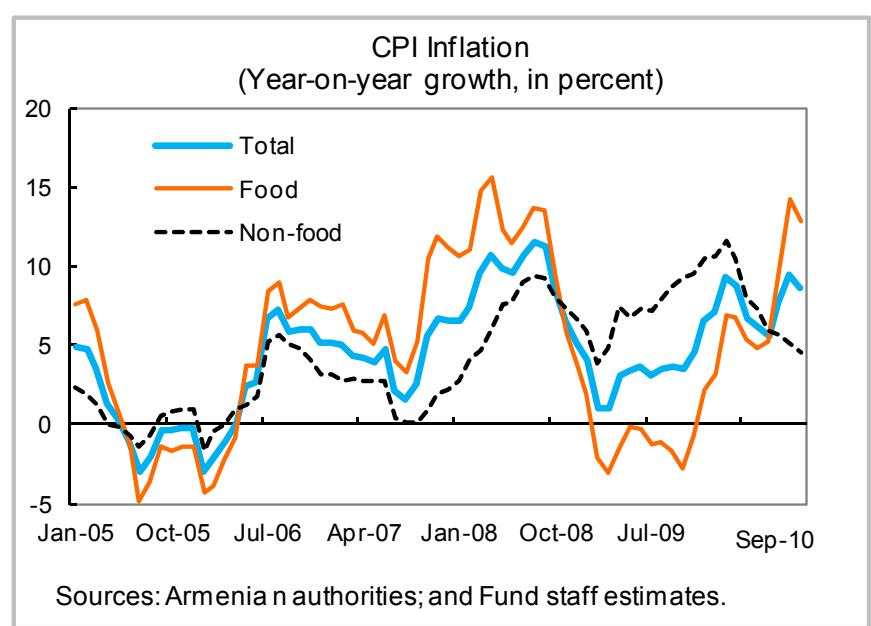
monetary and fiscal policies in place, inflation is expected to revert to the central bank's target of $4 \pm 1 \frac{1}{2}$ percent during the second half of 2011.

\section{Current account deficits are large, although public debt remains manageable} and private debt is negligible. The current account deficit was about 16 percent of GDP in 2009 and is projected to narrow to 14.6 percent of GDP in 2010. It has been financed mainly through donor assistance and foreign direct investment. Gross international reserves are expected to decline marginally to about 5 months of imports by year-end. Public debt is projected to increase sharply from 16 percent in 2008 to about 50 percent in 2012, but is set to decline steadily thereafter.

\section{The nominal exchange rate has} appreciated this year after depreciating by close to 35 percent between early 2009 and early 2010. The recent appreciation of about 10 percent reflects seasonal trends of real flows (e.g., lower food imports, higher tourism receipts), as well as some external borrowing from parent banks by domestic subsidiaries. Consistent with their intervention guidelines, the authorities have been leaning against the wind and have purchased about $\$ 70$ million net

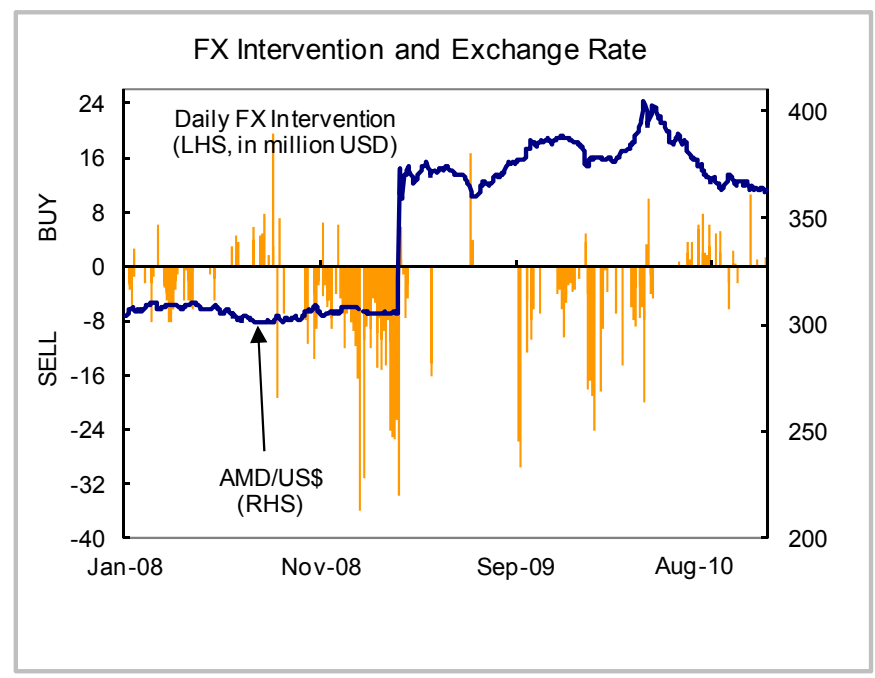


since end-March. These pressures were further exacerbated by requiring banks to have 50 percent — up from 25 percent — of their reserve requirements on foreign currency deposits to be maintained in drams (see below).

9. Monetary conditions have eased in recent months. While the announced policy rate has remained at 71/4 percent since May, liquidity conditions have eased owing mainly to dram injections from foreign exchange purchases by the Central Bank of Armenia (CBA), causing the overnight interbank rate to drop significantly below the policy rate. However, dram lending remains weak as long-term funding is scarce. As credit demand has returned,

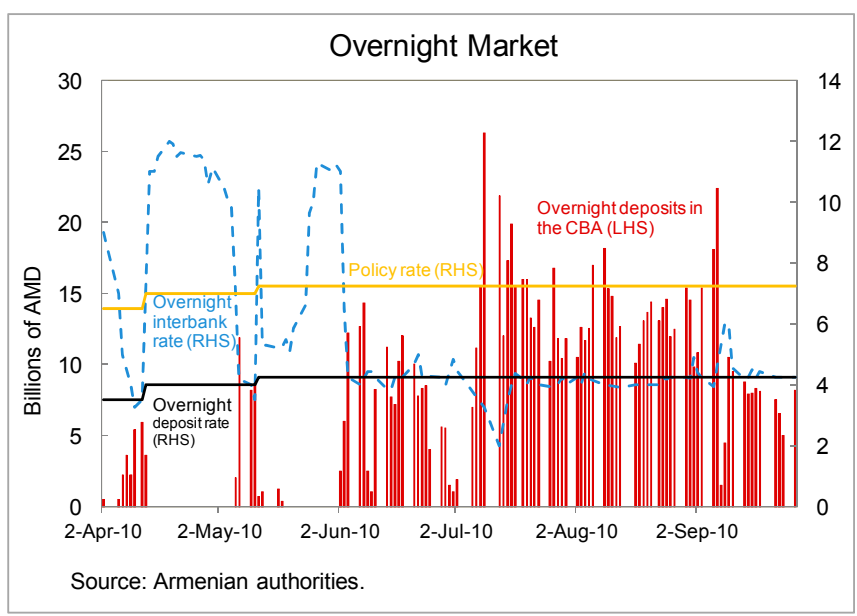
banks have begun to lend in foreign currency, using their large reserves.

10. Fiscal performance has improved markedly with the modest economic recovery this year. Year-over-year tax collection increased by 20 percent in the first eight months and the fiscal deficit is expected to fall by more than three percentage points of GDP this year.

\section{Facing a Sobering Outlook: Medium-Term Policy Challenges}

\section{While the economic and financial situation is improving, important} vulnerabilities have emerged or have become more evident. An undiversified economy marked by pervasive monopolies and closed borders, were well-known issues before the crisis, but were masked by the high growth rates. While the authorities' policy response and financial support from the international community have helped mitigate the crisis, public debt and current account deficits have risen sharply.

\section{Armenia now faces a much} more challenging outlook compared to the pre-crisis period. External demand is likely to remain weak. In particular, growth in Russia, Armenia's main trading partner and source of remittances and FDI, has moderated compared to previous years. Armenia is also vulnerable to developments in Europe, primarily due to lower demand for its commodity exports as well as the recent depreciation of the euro.

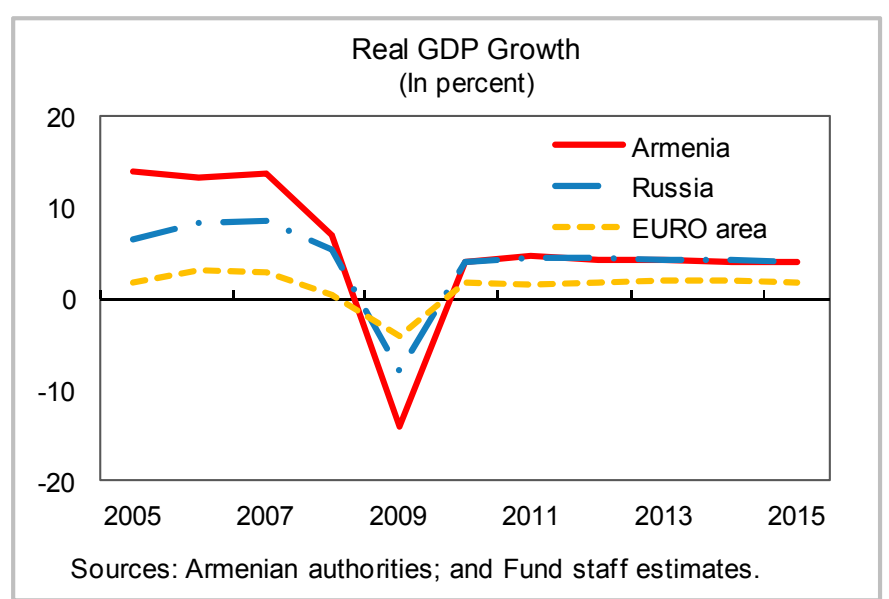


However, over the medium term, both exports and imports are expected to pick up with large investment projects and trade enhancing reforms ( $₫ 29$ and 30). In the short term, growth should pick up to 4.6 percent in 2011 , driven by base effects, particularly in agriculture. Over the medium term, GDP is likely to grow at around 4 percent annually. Given the wide range of shocks to which Armenia is exposed, risks are tilted to the downside.

13. In this setting, the $\mathbf{2 0 1 0}$ Article IV consultation focused on the policies needed to maintain fiscal and debt sustainability, support the adjustment to the external outlook, and ensure sustainable medium-term growth and poverty reduction.

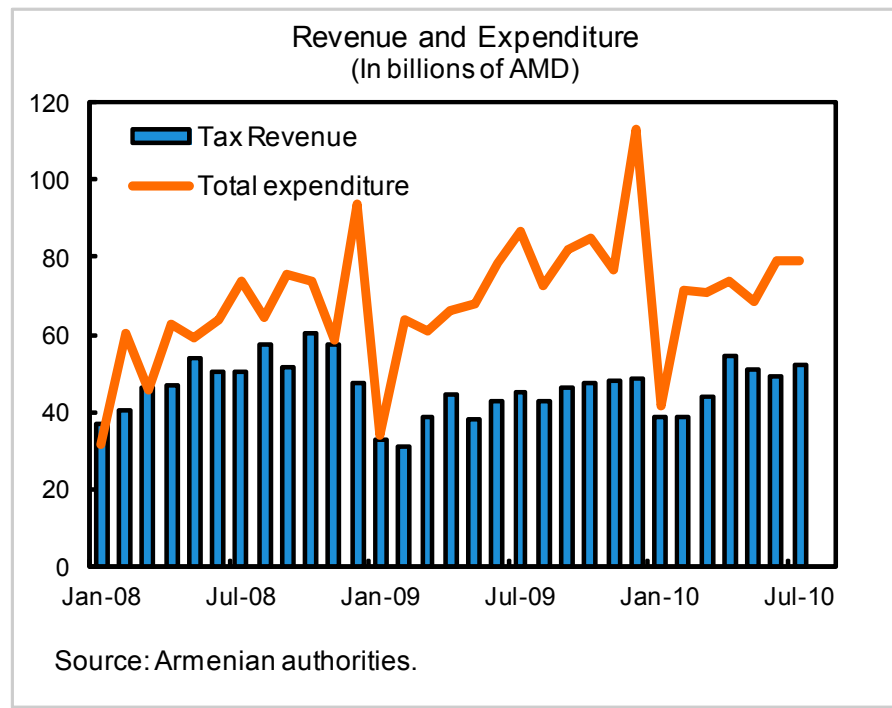

\section{REPORT ON THE DISCUSSIONS}

\section{A. Securing Sound Public Finances}

14. In response to the crisis, the authorities appropriately injected a large fiscal stimulus in 2009. Both the authorities and staff considered that the extent of the output contraction last year and the need to mitigate the impact of crisis on the poor warranted a sizable widening of the deficit. This helped sustain demand and ensure the provision of essential services. To the authorities' credit, most of the stimulus did not involve new recurrent expenditures or permanent tax reductions.

15. However, with the recovery of output and the rapid increase in public debt, fiscal consolidation is needed in the coming years. Public external debt, which was only 13.6 percent of GDP at end-2008, has since tripled and is expected to peak at about 42 percent of GDP in 2011. In addition, Armenia has a bunching of debt service obligations that rise to more than 20 percent of export receipts in 2013. The authorities are already

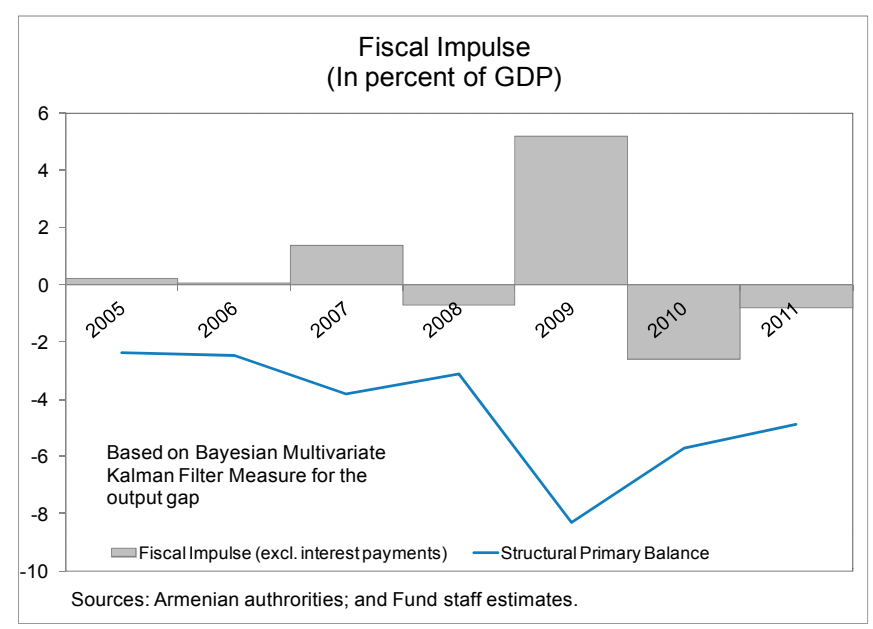


consolidating their fiscal accounts, with the deficit targeted to come down to 4.8 percent of GDP in 2010 from 8 percent in 2009. This implies an adjustment of more than 2 percentage points of GDP in the structural primary balance.

16. The authorities shared staff's debt sustainability concerns, and underscored their commitment to both prudent policies and revenue-enhancing reforms to help preserve public finances. The authorities noted that with external financing abating, higher deficits would require higher domestic financing and expose them to greater domestic rollover risk. Moreover, legislative provisions constrain the government's ability to borrow when public debt reaches 50 percent of GDP. Therefore, they intend to continue on a path of debt reduction, with the fiscal deficit slated to fall to about $2 \frac{1}{2}$ percent of GDP by 2013 . With this consolidation, Armenia's external debt will remain at a low risk of distress (Appendix I). Staff underscored that financing gaps will need to rely as much as possible on concessional financing to avoid a worsening of the debt dynamics.

\section{In addition to their wide-ranging tax reform agenda (described below),} strengthening public expenditure and debt management will be critical to achieving fiscal policy goals. The Medium-Term Expenditure Framework (MTEF) was re-introduced in August after having been suspended during the crisis. The updated MTEF is geared toward promoting growth, while placing public debt on a downward path and shifting spending toward social programs and investment in key sectors. To help identify areas for efficiency gains and savings, the authorities are looking forward to the IMF's technical assistance on expenditure rationalization in early 2011. Likewise, the authorities are strengthening their debt monitoring and planning capacity, and recently adopted a time-bound debt management strategy action plan, which aims to overhaul the debt recording system, modernize the internal organization of the debt unit, and enhance the primary debt market.

\section{The authorities and staff agreed} on the importance of protecting social spending. Social spending in Armenia is low, and the authorities recognize the need to protect the poor. To this end, allocations

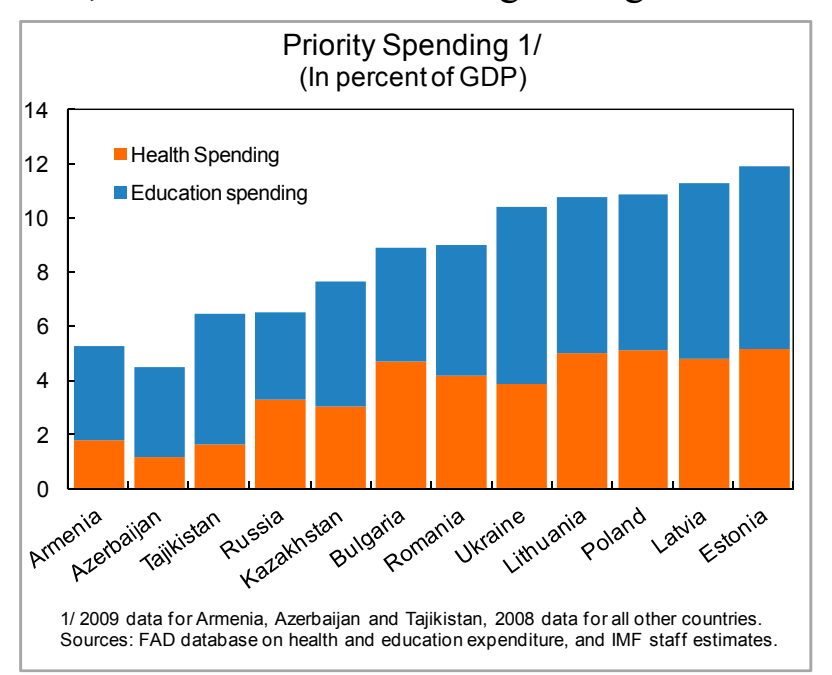


to the Family Benefits Program-Armenia's social safety net - will be raised by 15 percent in nominal terms in 2011 compared to 2010. The authorities have also agreed to gradually increase allocations for health spending and education, which, at 4 percent of GDP, remain low for Armenia's income level. Furthermore, the government will continue to provide support to the poor through other programs, including unemployment benefits and paid public works. $^{3}$

\section{B. Adjusting to the External Environment}

19. Following the global crisis, the external current account has worsened considerably. While the small foreign indebtedness of the household and corporate sector does not represent a vulnerability, an adverse shock to external inflows (e.g. remittances or public borrowing) could trigger an abrupt and disorderly adjustment in the current account. The real exchange rate is estimated to be overvalued by about 10-12 percent (Appendix II). Therefore, while the increased flexibility of the exchange rate and the fiscal retrenchment expected over the medium term are steps in the right direction, in staff's view, additional structural reform measures are needed to raise external competitiveness (Appendix IV, $₫ 29$ and 30) and bring the current account deficit to sustainable levels.

\section{The authorities agreed that competitiveness needs to be raised, but did not see} the exchange rate channel as the main avenue for achieving this goal. While they acknowledged that the real exchange rate was somewhat overvalued at present, they were concerned that a depreciation could lead to higher inflation. The authorities underscored that the exchange rate has been responding to market forces, and official interventions have been aimed at smoothing volatility in both directions and safeguarding reserves. Staff welcomed these two-way interventions, and stressed the importance of not resisting fundamental trends. Both staff and the authorities agreed that clear and transparent communication would be important in this regard (LOI $₫ 11$ ).

\footnotetext{
${ }^{3}$ After a sharp rise in outlays on these allowances in 2009 in connection with the crisis, increases in 2010 and beyond will be more moderate, with spending on allowances and pensions more generally declining in percent of GDP as the recovery proceeds, but remaining well above pre-crisis levels.
} 


\section{Monetary policy will continue to focus on price stability under the inflation}

targeting framework. In light of early signs of recovery, the authorities increased the policy rate by 225 basis points to $7 \frac{1}{4}$ percent from January through May. As the pace of recovery slowed in the second half of this year, the rate has been kept unchanged. While the current price pressures present a challenge, the authorities stressed - and staff agreed - that they are due to supply shocks to the agricultural sector and imported wheat prices, which are expected to recede gradually. So far, there has been little sign of a pass-through of price increases to wages, and staff agreed that demand pressures will remain low in the near term, given the fragile recovery and the negative output gap. Core inflation has also been on a declining trend since end-2009. In view of these considerations, a tightening of monetary policy is not warranted at this time. Nonetheless, staff recommended that the authorities remain vigilant and to stand ready to raise policy rates further if there is evidence of demand pressures or to prevent the entrenchment of second-round effects of temporary supply shocks on inflation.

\section{Strengthening the monetary} transmission mechanism will be a key element to raise the effectiveness of monetary policy. The authorities recognized the need to enhance the link between the policy rate and retail rates. However, market interest rates often diverge significantly from the policy rate for long periods, undermining efforts to increase its relevance. Staff urged the authorities to manage liquidity actively to keep market

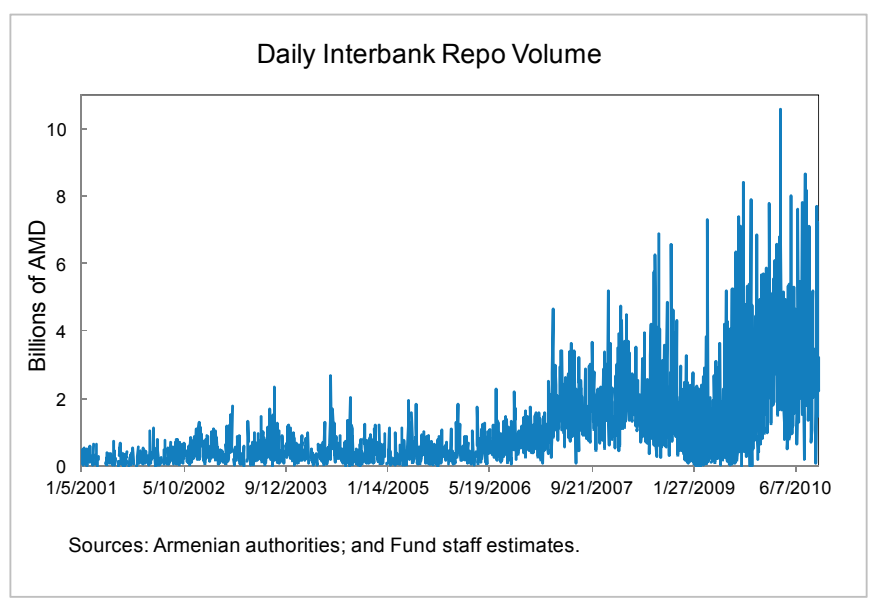
interest rates close to the policy rate. To enhance further the interest rate channel, the authorities are pursuing initiatives to develop the market for dram instruments, including standardizing the pricing and settlement of treasury bills in line with international practice and setting the legal groundwork for insurance and private investment funds to develop (LOI \12).

23. Measures to encourage dedollarization have helped at the margin, but a significant impact will ultimately hinge on a strong track record of macroeconomic policies and financial stability. The authorities noted that an increase, in several steps, in bank reserves on foreign currency deposits to be maintained in drams has widened interest rate spreads between dram and foreign currency deposits

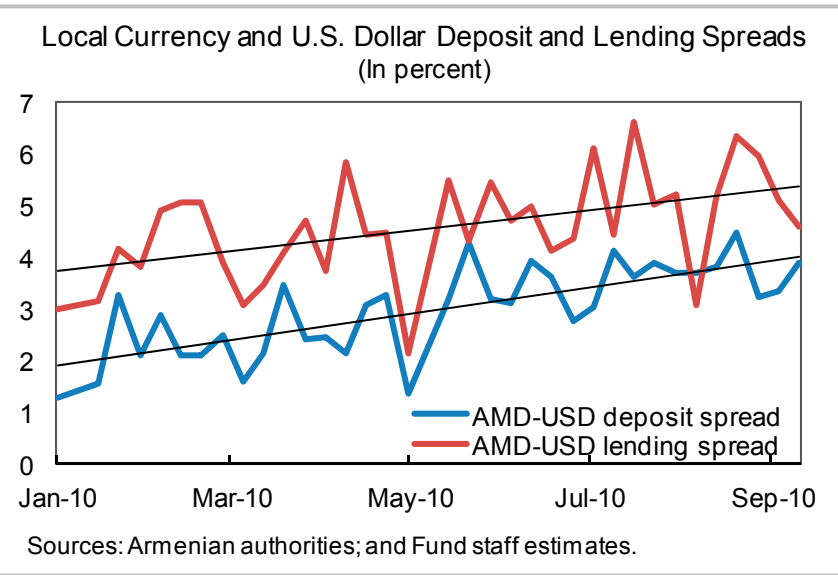


(LOI 913 ). Indeed the deposit dollarization rate has fallen slightly, although the recent appreciation of the exchange rate may have also played a role. Staff urged caution with introducing new reserve requirement norms, bearing in mind the trade-offs between prudential objectives and dedollarization. Ultimately, the credibility of monetary and exchange rate policies will be key to dedollarization.

\section{Creating a More Dynamic Economy}

24. The authorities reiterated their commitment to raise economic growth rates and reduce poverty in the medium term. To this end, a range of reforms to enhance stability and boost the competitiveness of the economy are envisaged.

\section{Tax reform}

25. Tax administration reforms remain crucial to the authorities' objectives of sound public finances and strengthening the business environment. With the tax-to-GDP ratio at about $16 \frac{1}{2}$ percent, persistently weak tax collection relative to comparators (Appendix III) undermines the authorities' ability to spend on priority areas such as investments in human and physical capital. At the same time, harassment by tax officials, reported by the private sector, undercuts the ability of firms to function efficiently. As outlined in the LOI ( $\$ 17)$, a number of steps have been implemented to boost oversight of the tax and customs agency, the SRC, including establishment of a board of appeals outside the purview of the SRC. Looking forward (LOI \18 and 19), improving VAT refund processing, expanding the large taxpayer unit, streamlining tax reporting requirements, and developing tax audit manuals should all go in the direction of lessening the burden of tax administration, reducing corruption and evasion, encouraging greater voluntary compliance, and enhancing revenue over the longer term.

\section{Regarding tax policy, efforts are underway to simplify the system and reduce}

distortions. The authorities will (i) abolish the presumptive taxation regime for petroleum products and move these goods to the regular taxation regime starting January 2011; (ii) harmonize excise tax rates for alcoholic beverages between domestic and imported products starting in 2011, and (iii) increase the presumptive payments on tobacco products starting in 2011, while gradually harmonizing taxation between domestic and imported tobacco and moving these goods from the presumptive to the regular taxation regime by 2014 (LOI $\rrbracket 20)$. 


\section{Strengthening the financial sector}

27. The banking sector remains stable, and the authorities are working toward reducing remaining vulnerabilities. The sector has been resilient to the strains of the last 18 months (as discussed in Appendix C of Country Report No. 10/97), and updated stress tests indicate that it is resilient to moderate interest rate, exchange rate, liquidity, and credit shocks. The authorities are continuing to enhance their risk management and supervisory frameworks, as well as crisis preparedness and contingency planning (LOI $\uparrow 15$ and 16).

28. Deepening financial intermediation, which remains low in Armenia, will be needed to support private sector growth and raise the traction of monetary policy. While private sector credit as a share of GDP has increased over the last decade, it stands at just over 20 percent of GDP. The authorities have requested a full FSAP update, given the importance of both the stability and development objectives.

\section{Structural reforms}

29. Several impediments inhibit Armenia's growth potential. As noted, Armenia's two closed borders clearly present a handicap, as does the oligopolistic structure of the private sector (Appendix IV). Furthermore, a majority of businesses indicate that corruption, access to finance, as well as crime, theft, and disorder have recently emerged as major concerns.

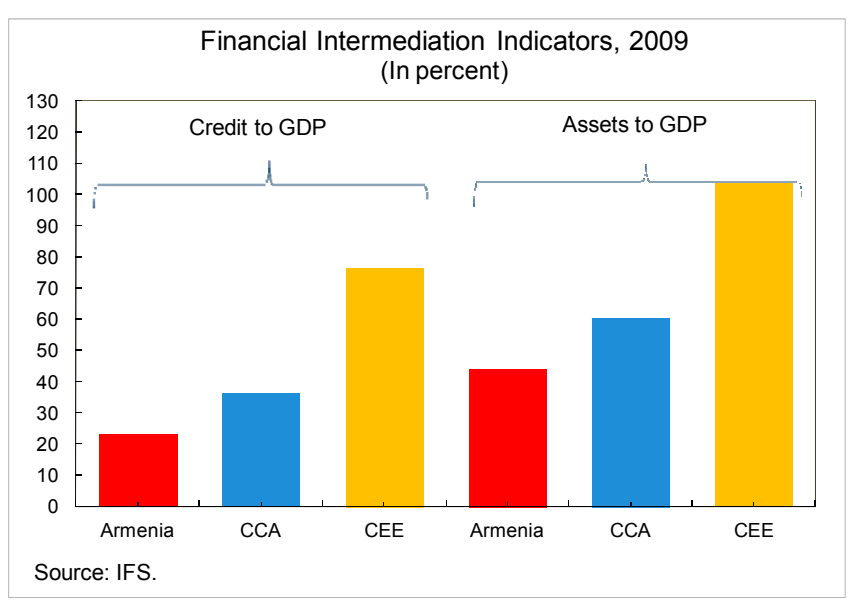

30. The authorities outlined a number of steps they are taking to improve the business climate. These involve introducing a legal restriction on public officials from engaging in commercial activities, strengthening the competition commission, and further reducing barriers to entry for new businesses, including a recent cabinet decree to create a one-stop shop for business registration.

31. Trade reform will also be critical, and a number of reforms are planned under the umbrella of the recently-launched negotiations for an EU Association Agreement with Armenia. Key steps include revised regulations and practices regarding standardization, certification, intellectual property rights, competition rules, and public procurement procedures. It is crucially important to reverse the recent decline in share of Armenian exports and the deteriorating role of the export sector. 

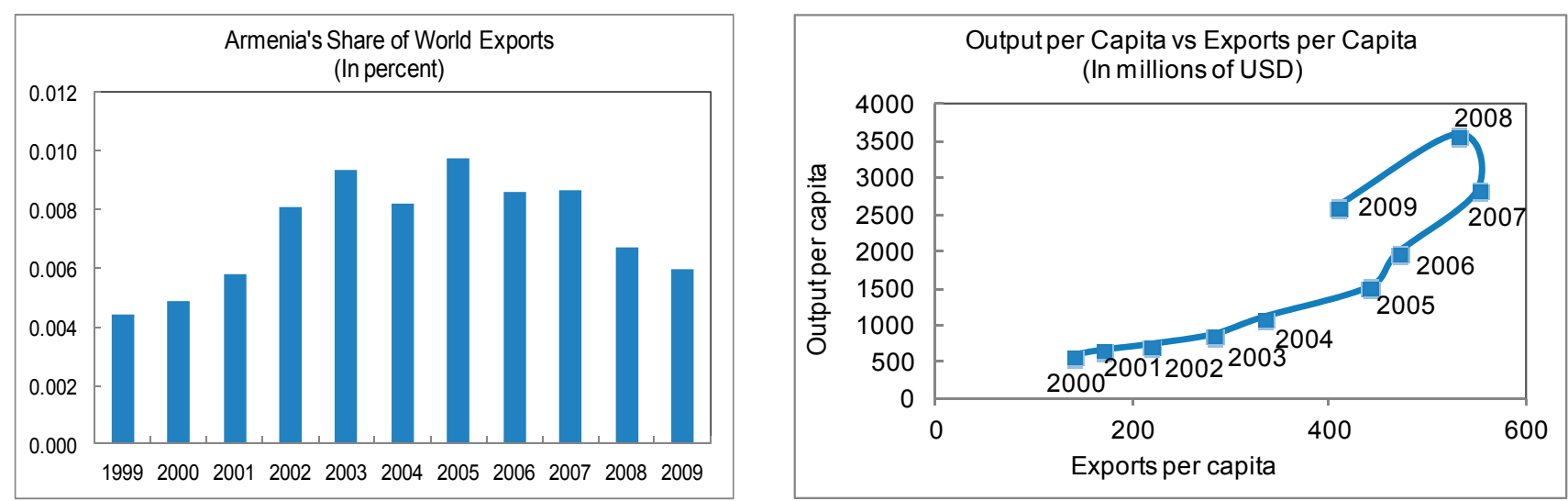

Sources: Armenian authorities; and Fund staff estimates.

\section{Poverty reduction}

32. Despite impressive gains over the last decade, the poverty rate remains high. While the targeting of social assistance programs has improved noticeably, coverage remains uneven with currently only 36 percent of the poor covered by the Family Benefits Program, a key element of social protection in Armenia. Although this represents an improvement over the coverage rate before the crisis, the authorities recognize that simplifying certification requirements and increasing outreach efforts could help achieve higher coverage.

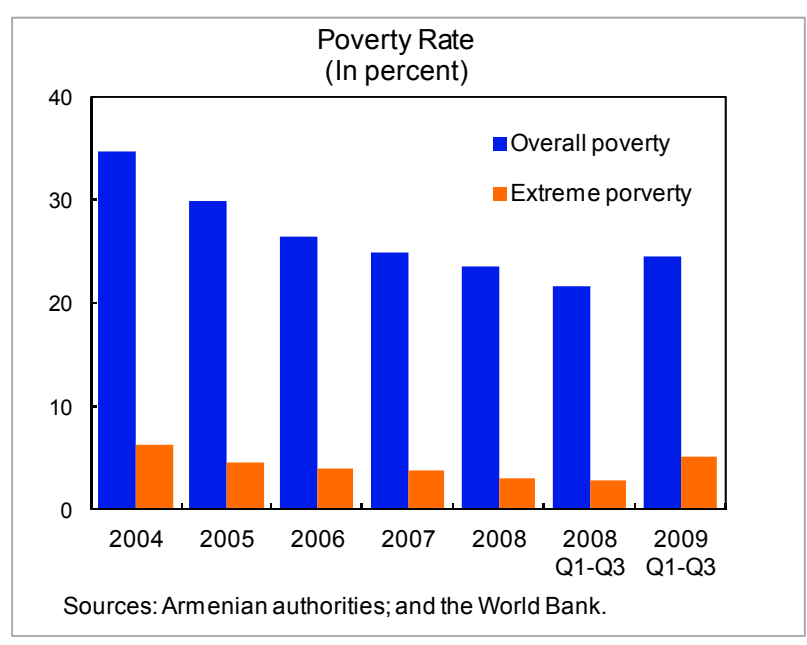

\section{The authorities will introduce an integrated system for the provision of social} protection services, in cooperation with the World Bank (LOI $\mid 25$ ). They are also in the process of updating their Poverty Reduction Strategy Paper. The authorities have continued the progress toward achieving the Millennium Development Goals, as indicated by lower maternal mortality rates, as well as an increase in life expectancy.

\section{The EFF/ECF-Supported Program Remains on TraCK}

34. Performance under the EFF/ECF-supported program has been strong. The program is on track, with all quantitative performance criteria and indicative targets for endJune 2010 and all but one structural benchmarks observed on time. The benchmark on establishing a tax appeals board was met with delay, in October, instead of September.

35. The authorities have requested minor modifications to the program. In particular, they have requested a modification of the end-December performance criteria for net 
international reserves, net domestic assets, and the program fiscal balance, largely to preserve the strong performance of net international reserves thus far this year and the postponement of some external budget financing to 2011. In addition, they have requested the establishment of performance criteria for end-June 2011.

36. While risks to the program exist, they are mitigated by several factors.

Policymakers face several challenges, particularly the need to unwind external imbalances, put fiscal and debt policies on sustainable path, move ahead with tax administration measures, and ensure sustainable growth and lower poverty. These risks are mitigated by the authorities' strong commitment, already demonstrated in managing the crisis well and in formulating a cohesive post-crisis reform program, a pre-crisis track record of sound macroeconomic management, and the resources made available to Armenia through the EFF/ECF arrangement.

37. Armenia's debt is expected to be sustainable in the medium term and its repayment capacity is good. Fund exposure is projected to peak at about 10 percent of GDP in 2011. Total debt service to the Fund would reach 14 percent of total exports in 2013, or about 3 percent of GDP.

38. The recently completed safeguards assessment found that the CBA continues to maintain a sound safeguards framework. Financial statements continue to be prepared and audited in accordance with international standards and published on a timely basis. A framework agreement between the CBA and the ministry of finance is in place on servicing budget support-related obligations to the Fund. The safeguards framework could be further improved by appointing external auditors for the CBA to a multi-year term, and strengthening its oversight arrangements by the inclusion of independent members on the Board and on the Audit Committee.

\section{Staff Appraisal}

39. The Armenian economy suffered a major setback during the global crisis with output contracting by more than 14 percent in 2009. The swift policy response of the authorities, with the assistance of the international community, rightly shifted to a countercyclical mode, dampening the impact of the crisis, especially on the most vulnerable segments of the population.

40. Armenia is on the right trajectory as it exits the crisis. Recent macroeconomic developments are encouraging, despite some-largely supply driven - inflationary pressures. Fiscal consolidation is moving forward, while the monetary policy stance remains neutral. Structural reforms have also progressed, but need to proceed faster to raise the growth potential of the economy.

41. Over the medium term, Armenia faces several challenges precipitated by the crisis. The external environment is difficult, and external imbalances must to be tackled 
expeditiously to avoid a disorderly adjustment. Decisive fiscal consolidation is needed to preserve debt sustainability and support medium-term external adjustment. Broader reforms are essential to promote growth and poverty reduction, namely, to address significant weaknesses in tax administration and the business environment, strengthen financial stability, and deepen financial intermediation.

42. The government's fiscal policy strategy appropriately addresses the mediumterm fiscal and debt vulnerabilities. With the economy gradually recovering, the fiscal stimulus is being gradually withdrawn. The budget deficit is projected to decline by 3 percent of GDP in 2010 and by a further 1 percent of GDP in 2011. The focus of consolidation is appropriately on enhancing revenue collection, rather than expenditure compression that could hurt spending on important sectors - education and health - as well as the poor. With these policies, the fiscal deficit is projected to fall to about 2 percent of GDP in the medium term, which will ensure that public debt remains sustainable. The MTEF and the new debt management strategy should substantially contribute to sound public finances.

43. The CBA's monetary policy response since the crisis has been appropriate. The move to a more flexible exchange rate, the gradual lowering of the policy rate during the crisis, and its gradual increase as the recovery started were steps in the right direction. In the near term, monetary policy should continue to remain vigilant, increasing the policy rate if demand pressures or second-round effects from food price shocks arise. The CBA should continue to improve its communications to ensure that inflation expectations remain anchored around the inflation target. Planned dedollarization efforts could facilitate the monetary transmission mechanism, but sustained and sound macroeconomic policies are essential to reduce dollarization.

44. The increased flexibility of the exchange rate is serving Armenia well. The authorities' commitment to maintaining a flexible exchange rate is welcome. The CBA intervention policy should continue to aim at only smoothing sharp fluctuations in the market, while guarding foreign exchange reserves and ensuring that the economy remains competitive. Any appreciation bias in the interventions will further contribute to a misalignment of the exchange rate with the equilibrium rate and delay external adjustment.

\section{Armenia's banking sector remains sound and proved resilient to the global} shocks. After rising in the first half of 2009, NPLs came down and have remained at low levels throughout 2010. Staff is encouraged by the recent pick up in lending to the private sector. It is essential that the authorities continue to implement measures to preserve financial stability, including strengthening the supervision framework, tightening foreign exchangerelated prudential regulations, and improving crisis preparedness and contingency planning.

46. Further improvements in tax administration will be needed to support fiscal consolidation and achieve stronger growth. While the government's commitment to reforming the tax administration is welcome, efforts to strengthen tax administration and 
advance tax policy reforms have had mixed success, in part reflecting political economy constraints. The recent actions are a step in the right direction, but decisive implementation in line with international best practices will be crucial to realize gains.

47. Structural reforms will be essential if Armenia is to enhance its competitiveness and raise its growth potential. Bolder and deeper reforms are needed to improve governance, enhance competition, diminish monopolistic behavior, diversify exports, and more generally, modernize the economy. Measures to address conflicts of interest of public officials, strengthen the competition framework, and reduce barriers to entry by firms are welcome and should be followed by further trade reforms.

48. Policies under the program remain on track. All quantitative performance criteria and indicative targets for end-June 2010 as well as all indicative targets for which data are available for end-September 2010 were observed. The structural benchmarks were also met on time, except for the benchmark on the establishment of tax appeals board, which was met with a one-month delay. With this strong performance, staff recommends completion of the first review under the EFF/ECF, approval of the request for modification of end-December 2010 performance criteria for net international reserves, net domestic assets, and the program fiscal balance, and the establishment of new performance criteria for end-June 2011.

49. It is proposed that the next Article IV consultation be held in accordance with the September 28, 2010 decision on consultation cycles. 
Armenia: Selected Economic and Financial Indicators, 2006-13

\begin{tabular}{|c|c|c|c|c|c|c|c|c|c|}
\hline & \multirow{2}{*}{$\begin{array}{r}2006 \\
\text { Act. }\end{array}$} & \multirow{2}{*}{$\begin{array}{r}2007 \\
\text { Act. }\end{array}$} & \multirow{2}{*}{$\begin{array}{r}2008 \\
\text { Act. }\end{array}$} & \multirow{2}{*}{$\frac{2009}{\text { Prel. }}$} & \multicolumn{2}{|l|}{2010} & \multirow{2}{*}{$\begin{array}{r}2011 \\
\text { Proj. }\end{array}$} & \multirow{2}{*}{$\begin{array}{l}2012 \\
\text { Proj. }\end{array}$} & \multirow{2}{*}{$\begin{array}{r}2013 \\
\text { Proj. }\end{array}$} \\
\hline & & & & & $\begin{array}{r}\text { Country Rep. } \\
\text { No. 10/223 }\end{array}$ & Proj. & & & \\
\hline \multicolumn{10}{|l|}{ National income and prices } \\
\hline Real GDP (percent change) & 13.2 & 13.7 & 6.9 & -14.2 & 4.8 & 4.0 & 4.6 & 4.3 & 4.2 \\
\hline Gross domestic product (in billions of drams) & 2,656 & 3,149 & 3,568 & 3,103 & 3,433 & 3,470 & 3,771 & 4,100 & 4,456 \\
\hline Gross domestic product (in millions of U.S. dollars) & 6,384 & 9,206 & 11,662 & 8,541 & 8,408 & 8,836 & 8,858 & 9,199 & 9,711 \\
\hline Gross domestic product per capita (in U.S. dollars) & 1,982 & 2,853 & 3,606 & 2,615 & 2,548 & 2,678 & 2,658 & 2,733 & 2,857 \\
\hline $\mathrm{CPI}$ (period average; percent change) & 2.9 & 4.4 & 9.0 & 3.5 & 6.7 & 7.8 & 5.5 & 4.6 & 4.0 \\
\hline CPI (end of period; percent change) & 5.2 & 6.6 & 5.2 & 6.6 & 6.2 & 7.1 & 4.6 & 4.0 & 4.0 \\
\hline GDP deflator (percent change) & 4.6 & 4.2 & 5.9 & 1.3 & 5.5 & 7.5 & 3.9 & 4.3 & 4.3 \\
\hline Poverty rate (in percent) $1 /$ & 26.5 & 25.0 & 23.5 & 24.6 & $\cdots$ & $\cdots$ & $\cdots$ & $\cdots$ & $\cdots$ \\
\hline \multicolumn{10}{|l|}{ Investment and saving (in percent of GDP) } \\
\hline Investment & 33.6 & 38.2 & 43.8 & 33.9 & 33.4 & 33.3 & 34.3 & 34.2 & 34.6 \\
\hline National savings & 31.7 & 31.8 & 32.0 & 17.9 & 19.5 & 18.7 & 21.7 & 22.6 & 24.4 \\
\hline \multicolumn{10}{|l|}{ Money and credit (end of period) } \\
\hline Reserve money (percent change) & 41.1 & 50.9 & 5.3 & 13.8 & 12.3 & 5.5 & 10.4 & $\ldots$ & ... \\
\hline Broad money (percent change) & 32.9 & 42.3 & 2.4 & 16.4 & 15.4 & 13.1 & 13.5 & $\ldots$ & $\ldots$ \\
\hline Velocity of broad money (end of period) & 5.5 & 4.6 & 5.0 & 3.8 & 3.6 & 3.7 & 3.6 & $\ldots$ & $\ldots$ \\
\hline Commercial banks' 3-month lending rate (in percent) & 17.1 & 18.6 & 17.9 & 19.1 & $\cdots$ & $\cdots$ & $\cdots$ & $\cdots$ & $\cdots$ \\
\hline \multicolumn{10}{|l|}{ Central government operations (in percent of GDP) } \\
\hline Revenue and grants & 18.0 & 20.1 & 20.5 & 21.1 & 22.2 & 21.8 & 22.7 & 21.8 & 22.2 \\
\hline Of which: tax revenue & 14.5 & 16.0 & 16.8 & 16.3 & 16.8 & 16.8 & 17.2 & 17.6 & 18.0 \\
\hline Expenditure 2/ & 20.0 & 22.4 & 22.2 & 28.9 & 26.9 & 26.6 & 26.6 & 24.7 & 24.6 \\
\hline Overall balance on a cash basis & -2.2 & -2.2 & -1.2 & -8.0 & -4.8 & -4.8 & -3.9 & -2.9 & -2.4 \\
\hline Government and government-guaranteed debt (in percent of GDP) & 18.7 & 16.1 & 16.2 & 40.6 & 48.2 & 44.1 & 48.9 & 46.5 & 42.9 \\
\hline Share of foreign currency debt (in percent) & 88.1 & 86.7 & 83.7 & 88.9 & 89.8 & 88.9 & 89.1 & 87.8 & 85.8 \\
\hline \multicolumn{10}{|l|}{ External sector } \\
\hline Exports of goods and services (in millions of U.S. dollars) & 1,510 & 1,777 & 1,757 & 1,336 & 1,505 & 1,513 & 1,720 & 1,927 & 2,124 \\
\hline Imports of goods and services (in millions of U.S. dollars) & $-2,536$ & $-3,589$ & $-4,748$ & $-3,683$ & $-3,833$ & $-3,889$ & $-4,129$ & $-4,383$ & $-4,653$ \\
\hline Exports of goods and services (percent change) & 6.7 & 17.6 & -1.1 & -24.0 & 15.0 & 13.3 & 13.7 & 12.0 & 10.2 \\
\hline Imports of goods and services (percent change) & 19.4 & 41.5 & 32.3 & -22.4 & 4.5 & 5.6 & 6.2 & 6.2 & 6.2 \\
\hline Current account balance (in percent of GDP) & -1.8 & -6.4 & -11.8 & -16.0 & -13.9 & -14.6 & -12.6 & -11.7 & -10.1 \\
\hline FDI (net, in millions of U.S. dollars) & 450 & 701 & 940 & 725 & 665 & 761 & 799 & 839 & 881 \\
\hline External debt (in percent of GDP) $3 /$ & 18.9 & 15.7 & 13.5 & 34.7 & 40.3 & 37.8 & 41.8 & 40.6 & 35.9 \\
\hline Debt service ratio (in percent of exports of goods and services) & 3.9 & 2.9 & 3.1 & 5.4 & 6.3 & 7.7 & 7.6 & 14.4 & 21.0 \\
\hline Gross international reserves (in millions of U.S. dollars) 4/ & 1,072 & 1,659 & 1,407 & 2,004 & 1,956 & 1,740 & 1,790 & 1,730 & 1,574 \\
\hline Import cover $5 /$ & 3.6 & 4.2 & 4.6 & 6.2 & 5.8 & 5.1 & 4.9 & 4.5 & 3.8 \\
\hline Nominal effective exchange rate (percent change) 6/ & 10.1 & 15.0 & 9.0 & -8.9 & $\ldots$ & $\cdots$ & $\cdots$ & $\cdots$ & ... \\
\hline Real effective exchange rate (percent change) 6/ & 9.1 & 15.3 & 11.5 & -6.0 & $\cdots$ & $\cdots$ & $\cdots$ & $\cdots$ & $\cdots$ \\
\hline End-of-period exchange rate (dram per U.S. dollar) & 364 & 304 & 307 & 378 & $\cdots$ & $\cdots$ & $\cdots$ & $\cdots$ & $\cdots$ \\
\hline Average exchange rate (dram per U.S. dollar) & 416 & 342 & 306 & 363 & $\ldots$ & $\ldots$ & $\ldots$ & $\ldots$ & $\ldots$ \\
\hline \multicolumn{10}{|l|}{ Memorandum item: } \\
\hline Population (in millions) & 3.2 & 3.2 & 3.2 & 3.2 & $\ldots$ & $\ldots$ & $\ldots$ & $\ldots$ & $\cdots$ \\
\hline
\end{tabular}

Sources: Armenian authorities; and Fund staff estimates and projections.

1/ For 2009, Q3 preliminary estimates.

$2 /$ Including the gas subsidy in 2006-08.

3/ Based on government and government-guaranteed debt.

4/ Excluding the special privatization account (SPA), but including the Russian project loan.

5 / Gross international reserves in months of next year's imports of goods and services, including the SDR holdings.

6/ A positive sign denotes appreciation. 
Table 2. Armenia: Balance of Payments, 2007-15

(In millions of U.S. dollars, unless otherwise indicated)

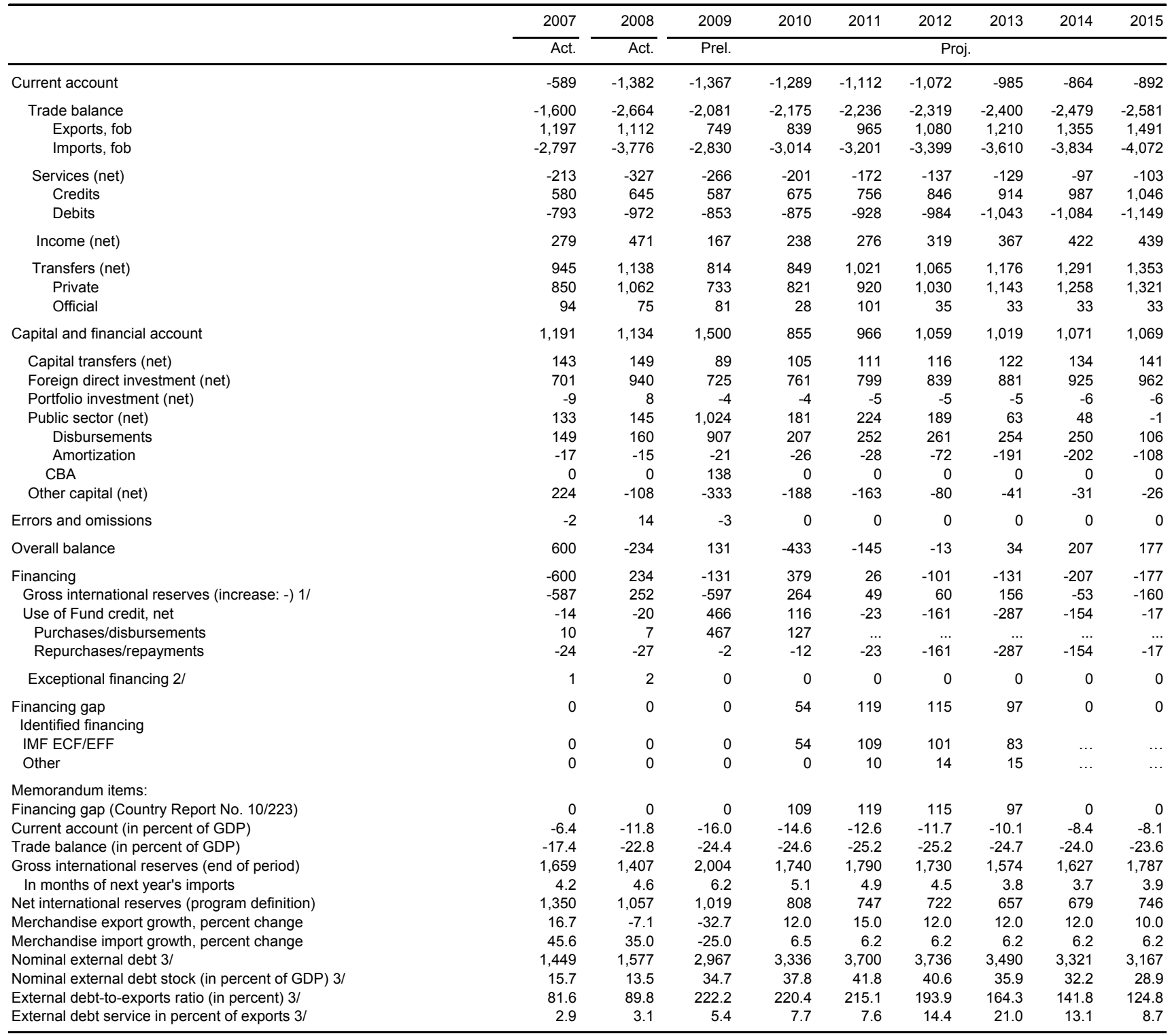

Sources: Armenian authorities; and Fund staff estimates and projections.

$1 /$ Gross international reserves include the SDR holdings.

2/ Debt relief from the United Kingdom through 2015 (in respect of IDA credits).

3 / Based on government and government-guaranteed debt. 
Table 3. Armenia: Monetary Accounts, 2007-11

(In billions of drams, unless otherwise indicated)

\begin{tabular}{|c|c|c|c|c|c|c|c|c|c|c|c|}
\hline & \multirow{2}{*}{$\begin{array}{r}2007 \\
\text { Dec. } \\
\text { Act. }\end{array}$} & \multirow{2}{*}{$\begin{array}{r}2008 \\
\text { Dec. } \\
\text { Act. }\end{array}$} & \multirow{2}{*}{$\begin{array}{r}2009 \\
\text { Dec. } \\
\text { Act. }\end{array}$} & \multicolumn{4}{|c|}{2010} & \multicolumn{4}{|c|}{2011} \\
\hline & & & & $\begin{array}{l}\text { Mar. } \\
\text { Prel. }\end{array}$ & $\frac{\text { Jun. }}{\text { Prel. }}$ & $\begin{array}{r}\text { Sep. } \\
\text { Prog. } 1 /\end{array}$ & $\begin{array}{r}\text { Dec. } \\
\text { Prog. } 1 /\end{array}$ & $\begin{array}{r}\text { Mar. } \\
\text { Prog. } 1 /\end{array}$ & $\begin{array}{r}\text { Jun. } \\
\text { Prog. } 1 /\end{array}$ & $\begin{array}{r}\text { Sep. } \\
\text { Prog. } 11\end{array}$ & $\begin{array}{r}\text { Dec. } \\
\text { Prog. 11 }\end{array}$ \\
\hline \multicolumn{12}{|l|}{ Central Bank of Armenia } \\
\hline Net foreign assets & 445.9 & 377.5 & 515.4 & 503.3 & 415.0 & 442.6 & 411.4 & 419.6 & 412.9 & 420.1 & 409.7 \\
\hline Net international reserves & 456.2 & 390.0 & 595.8 & 585.6 & 487.6 & 515.9 & 495.5 & 504.4 & 498.5 & 506.6 & 496.9 \\
\hline Medium and long-term & -10.3 & -12.4 & -80.5 & -82.3 & -72.6 & -73.4 & -84.1 & -84.9 & -85.7 & -86.4 & -87.2 \\
\hline Net domestic assets & -18.8 & 72.2 & -3.7 & -38.3 & 19.6 & 29.0 & 128.5 & 95.5 & 117.5 & 134.7 & 186.3 \\
\hline Claims on general government (net) & -89.8 & -96.5 & -152.5 & -154.5 & -145.6 & -126.5 & -90.0 & -75.3 & -62.2 & -59.5 & -64.5 \\
\hline Of which: central government (net) & -51.4 & -84.4 & -138.8 & -132.3 & -118.0 & -98.9 & -62.4 & -47.7 & -34.6 & -31.9 & -36.8 \\
\hline Claims on banks & 24.6 & 80.6 & 66.2 & 59.5 & 55.4 & 76.6 & 137.6 & 89.8 & 98.8 & 113.2 & 169.8 \\
\hline $\mathrm{KfW}$ & 14.4 & 19.9 & 39.1 & 43.5 & 49.8 & 50.6 & 61.3 & 62.1 & 62.9 & 63.6 & 64.4 \\
\hline Monetary instruments (net) excluding CBA bills & 10.2 & 60.7 & 27.1 & 16.0 & 5.5 & 26.0 & 76.3 & 27.7 & 35.9 & 49.6 & 105.4 \\
\hline CBA bills $2 /$ & -49.0 & -1.4 & 0.0 & 0.0 & 0.0 & 0.0 & 0.0 & 0.0 & 0.0 & 0.0 & 0.0 \\
\hline Other items (net) & 95.3 & 89.5 & 82.6 & 56.7 & 109.8 & 78.9 & 80.9 & 81.0 & 81.0 & 81.0 & 80.9 \\
\hline Reserve money & 427.1 & 449.7 & 511.7 & 465.0 & 434.5 & 471.6 & 540.0 & 515.0 & 530.3 & 554.8 & 595.9 \\
\hline Currency issue & 350.3 & 344.5 & 320.7 & 276.4 & 295.6 & 329.3 & 377.7 & 350.3 & 361.8 & 378.1 & 417.6 \\
\hline Deposits & 76.8 & 105.2 & 191.0 & 188.6 & 139.0 & 142.3 & 162.3 & 164.7 & 168.6 & 176.7 & 178.4 \\
\hline Deposits in drams & 38.4 & 39.3 & 42.3 & 31.2 & 41.8 & 57.2 & 61.4 & 62.8 & 63.6 & 64.7 & 65.7 \\
\hline Deposits in foreign currency & 38.5 & 65.9 & 148.7 & 157.4 & 97.2 & 85.1 & 100.9 & 101.9 & 105.0 & 112.0 & 112.7 \\
\hline \multicolumn{12}{|l|}{ Banking system } \\
\hline Net foreign assets & 369.6 & 229.2 & 379.1 & 380.9 & 285.2 & 295.4 & 292.0 & 255.1 & 238.4 & 209.7 & 209.2 \\
\hline Net domestic assets & 321.8 & 479.0 & 444.9 & 444.0 & 510.3 & 558.1 & 640.0 & 677.1 & 716.2 & 777.8 & 848.5 \\
\hline Claims on government (net) & -54.0 & -37.3 & -125.3 & -145.1 & -142.5 & -101.7 & -60.0 & -44.0 & -30.2 & -14.8 & -5.5 \\
\hline Of which: claims on central government (net) & -15.6 & -25.3 & -111.6 & -122.8 & -114.9 & -74.1 & -32.3 & -16.4 & -2.6 & 12.8 & 22.2 \\
\hline Claims on rest of the economy & 429.8 & 638.6 & 728.3 & 771.8 & 785.7 & 821.7 & 866.8 & 887.9 & 913.2 & 959.4 & 1020.8 \\
\hline Other items (net) & -54.0 & -122.3 & -158.0 & -182.7 & -132.9 & -161.8 & -166.8 & -166.8 & -166.8 & -166.8 & -166.8 \\
\hline Broad money & 691.3 & 708.2 & 824.0 & 824.9 & 795.5 & 853.6 & 932.0 & 932.2 & 954.6 & 987.6 & 1057.8 \\
\hline Currency in circulation & 326.0 & 316.1 & 282.7 & 247.5 & 265.2 & 292.3 & 338.7 & 317.3 & 328.8 & 345.1 & 387.6 \\
\hline Deposits & 365.3 & 392.2 & 541.3 & 577.4 & 530.3 & 561.3 & 593.3 & 614.8 & 625.8 & 642.5 & 670.2 \\
\hline Domestic currency & 235.0 & 219.5 & 170.7 & 157.4 & 165.9 & 177.1 & 189.7 & 197.2 & 201.9 & 208.9 & 223.7 \\
\hline Foreign currency & 130.3 & 172.6 & 370.6 & 420.0 & 364.4 & 384.2 & 403.6 & 417.7 & 423.9 & 433.6 & 446.5 \\
\hline \multicolumn{12}{|l|}{ Memorandum items: } \\
\hline Exchange rate (in drams per U.S. dollar, end of period) & 304.2 & 306.7 & 377.9 & 400.5 & 367.5 & & $\ldots$ & $\ldots$ & $\ldots$ & $\ldots$ & \\
\hline NIR, program definition, at program exchange rates (in millions of U.S. dollars) & $1,350.1$ & $1,056.6$ & $1,019.0$ & 895.3 & 912.4 & 898.3 & 808.4 & 830.6 & 779.9 & 800.8 & 746.8 \\
\hline 12-month change in reserve money (in percent) & 50.9 & 5.3 & 13.8 & 18.7 & 6.5 & 2.1 & 5.5 & 10.8 & 22.0 & 17.7 & 10.4 \\
\hline 12-month change in broad money (in percent) & 42.3 & 2.4 & 16.4 & 28.6 & 19.2 & 13.0 & 13.1 & 13.0 & 20.0 & 15.7 & 13.5 \\
\hline 12-month change in private sector credit (in percent) & 78.0 & 48.6 & 14.0 & 17.0 & 24.6 & 20.5 & 19.0 & 15.0 & 16.2 & 16.8 & 17.8 \\
\hline Velocity of broad money (end of period) & 4.6 & 5.0 & 3.8 & 3.8 & 4.1 & 4.0 & 3.7 & 3.8 & 3.8 & 3.7 & 3.6 \\
\hline Money multiplier & 1.6 & 1.6 & 1.6 & 1.8 & 1.8 & 1.8 & 1.7 & 1.8 & 1.8 & 1.8 & 1.8 \\
\hline Dollarization in bank deposits $3 /$ & 35.7 & 44.0 & 68.5 & 72.7 & 68.7 & 68.4 & 68.0 & 67.9 & 67.7 & 67.5 & 66.6 \\
\hline Dollarization in broad money 4/ & 18.9 & 24.4 & 45.0 & 50.9 & 45.8 & 45.0 & 43.3 & 44.8 & 44.4 & 43.9 & 42.2 \\
\hline Currency in circulation in percent of deposits & 89.2 & 80.6 & 52.2 & 42.9 & 50.0 & 52.1 & 57.1 & 51.6 & 52.5 & 53.7 & 57.8 \\
\hline Stock of foreign currency deposits (in millions of U.S. dollars) $1 /$ & 428.4 & 562.8 & 980.8 & $1,091.0$ & 946.5 & 997.8 & $1,048.3$ & $1,084.9$ & $1,101.1$ & $1,126.3$ & $1,159.6$ \\
\hline Banking system financing of the central government (cumulative) $5 /$ & -24.7 & -9.6 & -86.4 & -11.2 & -3.2 & 37.5 & 79.3 & 16.0 & 29.8 & 45.2 & 54.5 \\
\hline
\end{tabular}

Sources: Central Bank of Armenia; and Fund staff estimates and projections.

1/ At the program exchange rate.

2/ Following the agreement between the CBA and the Ministry of Finance, the issue of new CBA bills was terminated in 2008.

3/ Ratio of foreign currency deposits to total deposits (in percent).

5/ Discrepancy between the fiscal and monetary accounts in 2009Q3-Q4 and 2010 is exp lained by government lending to the economy through commercial banks. 
Table 4. Armenia: Financial Soundness Indicators for the Banking Sector, 2005-10

(In percent, unless otherwise indicated)

\begin{tabular}{|c|c|c|c|c|c|c|c|c|c|c|c|}
\hline & \multirow{2}{*}{$\begin{array}{r}2005 \\
\text { Dec. }\end{array}$} & \multirow{2}{*}{$\begin{array}{r}2006 \\
\text { Dec. }\end{array}$} & \multirow{2}{*}{$\begin{array}{r}2007 \\
\text { Dec. }\end{array}$} & \multirow{2}{*}{$\begin{array}{r}2008 \\
\text { Dec. }\end{array}$} & \multicolumn{4}{|c|}{2009} & \multicolumn{3}{|c|}{2010} \\
\hline & & & & & Mar. & Jun. & Sep. & Dec. & Mar. & Jun. & Aug. \\
\hline \multicolumn{12}{|l|}{ Capital adequacy } \\
\hline Total regulatory capital to risk-weighted assets & 33.7 & 34.9 & 30.1 & 27.5 & 26.1 & 28.0 & 28.3 & 28.3 & 28.6 & 28.9 & 28.4 \\
\hline Tier I regulatory capital to risk-weighted assets & 31.7 & 32.7 & 29.0 & $\ldots$ & 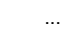 & $\ldots$ & $\ldots$ & $\ldots$ & $\ldots$ & $\ldots$ & \\
\hline Capital (net worth) to assets & 21.5 & 22.9 & 22.5 & 23.0 & 21.2 & 22.3 & 21.0 & 21.0 & 20.6 & 22.1 & 21.2 \\
\hline \multicolumn{12}{|l|}{ Asset composition } \\
\hline \multicolumn{12}{|l|}{ Sectoral distribution of loans (in billions of drams) } \\
\hline Industry (excluding energy sector) & 30.4 & 34.3 & 49.1 & 66.2 & 78.0 & 76.0 & 89.6 & 115.3 & 121.2 & 127.7 & 129.1 \\
\hline Energy sector & 8.7 & 5.0 & 7.2 & 19.7 & 17.3 & 18.6 & 26.7 & 31.7 & 32.0 & 24.3 & 33.3 \\
\hline Agriculture & 11.3 & 14.2 & 22.4 & 36.5 & 44.6 & 45.9 & 45.2 & 44.2 & 48.1 & 48.7 & 50.5 \\
\hline Construction & 7.9 & 11.5 & 22.0 & 39.5 & 43.3 & 44.0 & 48.9 & 54.1 & 57.5 & 58.8 & 59.3 \\
\hline Transport and communication & 3.7 & 3.9 & 5.8 & 10.2 & 10.3 & 9.8 & 14.7 & 15.4 & 17.8 & 22.2 & 25.7 \\
\hline $\begin{array}{l}\text { Trade/commerce } \\
\text { Sectoral distribution of loans to total loans (percent of total) }\end{array}$ & 42.2 & 49.6 & 86.8 & 132.0 & 140.4 & 131.0 & 143.6 & 145.5 & 161.0 & 164.7 & 167.0 \\
\hline Industry (excluding energy sector) & 17.0 & 16.2 & 11.9 & 10.7 & 12.2 & 12.5 & 13.7 & 16.5 & 16.2 & 16.9 & 16.6 \\
\hline Energy sector & 4.8 & 2.3 & 1.7 & 3.2 & 2.7 & 3.1 & 4.1 & 4.5 & 4.3 & 3.2 & 4.3 \\
\hline Agriculture & 6.3 & 6.7 & 5.4 & 5.9 & 7.0 & 7.6 & 6.9 & 6.3 & 6.4 & 6.4 & 6.5 \\
\hline Construction & 4.4 & 5.5 & 5.3 & 6.4 & 6.8 & 7.3 & 7.5 & 7.7 & 7.7 & 7.8 & 7.6 \\
\hline Transport and communication & 2.0 & 1.9 & 1.4 & 1.7 & 1.6 & 1.6 & 2.2 & 2.2 & 2.4 & 2.9 & 3.3 \\
\hline Trade/commerce & 23.5 & 23.4 & 21.1 & 21.4 & 22.0 & 21.6 & 22.0 & 20.8 & 21.6 & 21.8 & 21.4 \\
\hline Foreign exchange loans to total loans & 63.7 & 52.8 & 40.7 & 38.7 & 48.7 & 51.5 & 54.3 & 54.0 & 54.3 & 55.4 & 56.1 \\
\hline \multicolumn{12}{|l|}{ Asset quality } \\
\hline Nonperforming loans (in billions of drams) & 3.8 & 6.0 & 10.3 & 27.9 & 50.2 & 64.6 & 64.5 & 36.3 & 43.9 & 38.5 & 40.3 \\
\hline Watch (up to 90 days past due) & 2.9 & 3.6 & 5.8 & 21.8 & 36.6 & 38.8 & 27.0 & 14.9 & 23.2 & 18.3 & 20.5 \\
\hline Substandard (91-180 days past due) & 0.5 & 1.0 & 1.3 & 3.4 & 11.4 & 19.3 & 23.2 & 10.1 & 16.0 & 13.8 & 15.1 \\
\hline Doubtful (181-270 days past due) & 0.5 & 1.4 & 3.1 & 2.7 & 2.1 & 6.5 & 14.3 & 11.3 & 4.7 & 6.4 & 4.6 \\
\hline Loss (>270 days past due) & $\ldots$ & $\ldots$ & & 10.2 & 12.7 & 14.3 & 20.9 & 26.2 & 32.3 & 32.0 & 35.2 \\
\hline Nonperforming loans to gross loans & 1.9 & 2.5 & 2.4 & 4.4 & 7.6 & 10.2 & 9.3 & 4.8 & 5.6 & 4.8 & 4.9 \\
\hline Provisions to nonperforming loans & 70.7 & 64.3 & 66.6 & 38.2 & 26.9 & 26.6 & 33.1 & 46.7 & 36.2 & 41.4 & 39.3 \\
\hline Spread between highest and lowest rates of interbank borrowing in AMD & 3.7 & 3.0 & 0.5 & 3.0 & 4.5 & 5.0 & 7.0 & 2.5 & 3.0 & 2.8 & 4.0 \\
\hline Spread between highest and lowest rates of interbank borrowing in foreign currency & 1.0 & 2.3 & 0.0 & 1.0 & 8.0 & 11.3 & 5.8 & 3.0 & 4.0 & 7.6 & 0.2 \\
\hline \multicolumn{12}{|l|}{ Earnings and profitability } \\
\hline ROA (profits to period average assets) & 3.1 & 3.6 & 2.9 & 3.1 & -0.1 & -0.1 & 0.0 & 0.7 & 1.3 & 1.8 & 1.8 \\
\hline ROE (profits to period average equity) & 15.5 & 15.9 & 14.9 & 13.6 & -0.7 & -0.3 & 0.1 & 3.4 & 6.1 & 8.5 & 8.3 \\
\hline Interest margin to gross income & 41.1 & 47.1 & 47.9 & 45.9 & 47.1 & 44.4 & 42.2 & 42.2 & 43.5 & 44.1 & 44.1 \\
\hline Interest income to gross income & 59.8 & 66.3 & 68.6 & 72.7 & 86.2 & 83.0 & 79.5 & 78.3 & 79.0 & 78.9 & 78.4 \\
\hline Noninterest expenses to gross income & 49.7 & 45.1 & 44.5 & 42.0 & 45.3 & 44.1 & 41.5 & 40.7 & 39.4 & 39.6 & 39.2 \\
\hline \multicolumn{12}{|l|}{ Liquidity } \\
\hline Liquid assets to total assets & 44.2 & 41.2 & 33.7 & 23.8 & 29.1 & 32.0 & 35.2 & 34.2 & 33.9 & 30.1 & 29.5 \\
\hline Liquid assets to total short-term liabilities & 110.5 & 106.1 & 98.2 & 103.1 & 135.4 & 145.3 & 146.2 & 142.1 & 140.4 & 128.8 & 129.0 \\
\hline Customer deposits to total (non-interbank) loans & 140.5 & 130.8 & 106.2 & 81.4 & 92.1 & 96.3 & 100.7 & 96.4 & 96.5 & 86.8 & 87.4 \\
\hline Foreign exchange liabilities to total liabilities & 66.8 & 56.0 & 48.3 & 55.1 & 69.4 & 70.9 & 68.9 & 67.6 & 70.3 & 67.4 & 66.4 \\
\hline \multicolumn{12}{|l|}{ Sensitivity to market risk } \\
\hline Gross open positions in foreign exchange to capital & 4.9 & 4.4 & 8.8 & 11.5 & 13.8 & 8.4 & 7.1 & 3.4 & 3.1 & 3.8 & 2.8 \\
\hline
\end{tabular}

Source: Central Bank of Armenia. 
Table 5. Armenia: Central Government Operations, 2008-13

(In billions of drams)

\begin{tabular}{|c|c|c|c|c|c|c|c|c|c|c|c|c|c|c|c|}
\hline & \multirow{3}{*}{$\begin{array}{l}2008 \\
\text { Act. }\end{array}$} & \multirow{3}{*}{$\begin{array}{l}2009 \\
\text { Prel. }\end{array}$} & \multicolumn{6}{|c|}{2010} & \multicolumn{5}{|c|}{2011} & \multirow[t]{2}{*}{2012} & \multirow[t]{2}{*}{2013} \\
\hline & & & \multirow{2}{*}{\multicolumn{2}{|c|}{ Proj. $\begin{array}{c}\text { Country Rep. } \\
\text { No. 10/223 }\end{array}$}} & Q1 & Q2 & Q3 & \multirow{2}{*}{$\frac{Q 4}{\text { Proj. }}$} & \multirow[b]{2}{*}{ Proj. } & \multirow{2}{*}{$\begin{array}{l}\text { Q1 } \\
\text { Proj. }\end{array}$} & \multirow{2}{*}{$\begin{array}{l}\text { Q2 } \\
\text { Proj. }\end{array}$} & Q3 & $\underline{Q 4}$ & & \\
\hline & & & & & Prel. & Prel. & Proj. & & & & & Proj. & Proj. & Proj. & Proj. \\
\hline Total revenue and grants & 730.8 & 655.6 & 755.3 & 761.2 & 158.0 & 197.8 & 199.8 & 199.8 & 855.2 & 176.5 & 225.9 & 225.5 & 227.3 & 895.1 & 989.9 \\
\hline Total revenue & 715.6 & 634.4 & 712.7 & 706.4 & 153.2 & 192.2 & 186.5 & 180.8 & 791.9 & 170.0 & 212.9 & 207.5 & 201.6 & 877.9 & 972.9 \\
\hline Tax revenues & 597.9 & 505.9 & 583.1 & 576.8 & 120.8 & 154.7 & 155.6 & 152.0 & 650.2 & 134.6 & 172.4 & 173.2 & 170.0 & 723.5 & 804.1 \\
\hline VAT & 296.3 & 239.2 & 275.6 & 272.7 & 57.5 & 75.1 & 77.8 & 65.3 & 300.9 & 62.7 & 82.0 & 84.9 & 71.3 & 334.8 & 368.1 \\
\hline Profits, simplified and presumptive & 113.3 & 104.9 & 110.5 & 109.3 & 23.9 & 30.3 & 26.0 & 30.2 & 123.3 & 26.7 & 33.8 & 29.0 & 33.7 & 137.1 & 152.4 \\
\hline Personal income tax & 53.7 & 60.2 & 69.4 & 68.6 & 16.2 & 18.3 & 18.0 & 16.9 & 77.4 & 18.1 & 20.4 & 20.1 & 18.9 & 86.1 & 95.7 \\
\hline Customs duties & 37.3 & 25.1 & 34.2 & 33.8 & 6.7 & 8.5 & 8.0 & 10.9 & 44.6 & 8.7 & 11.1 & 10.5 & 14.3 & 49.7 & 55.2 \\
\hline Other & 97.3 & 76.5 & 93.3 & 92.3 & 16.5 & 22.5 & 25.7 & 28.6 & 104.1 & 18.4 & 25.1 & 28.7 & 31.9 & 115.8 & 132.7 \\
\hline Social contributions & 104.1 & 102.9 & 105.2 & 105.2 & 26.2 & 28.5 & 26.9 & 23.7 & 120.4 & 30.0 & 32.6 & 30.8 & 27.1 & 133.2 & 147.7 \\
\hline Other revenue & 13.6 & 25.6 & 24.4 & 24.4 & 6.2 & 9.0 & 4.0 & 5.1 & 21.3 & 5.4 & 7.9 & 3.5 & 4.5 & 21.2 & 21.1 \\
\hline Grants & 15.1 & 21.2 & 42.7 & 54.8 & 4.8 & 5.5 & 13.3 & 19.0 & 63.2 & 6.5 & 13.0 & 18.1 & 25.7 & 17.3 & 17.0 \\
\hline Total expenditure & 793.4 & 897.1 & 923.2 & 924.3 & 188.8 & 224.3 & 246.1 & 264.0 & 1003.0 & 208.1 & 261.2 & 263.5 & 270.1 & 1013.1 & 1095.1 \\
\hline Expense & 652.0 & 712.0 & 754.3 & 754.3 & 152.1 & 182.1 & 191.5 & 228.5 & 803.4 & 165.5 & 200.9 & 203.1 & 233.9 & 818.3 & 889.4 \\
\hline Wages & 73.0 & 83.5 & 87.3 & 87.3 & 17.4 & 20.3 & 23.1 & 26.5 & 89.5 & 17.8 & 20.8 & 23.7 & 27.1 & 92.2 & 101.5 \\
\hline Pensions & 4.1 & 4.6 & 4.8 & 4.8 & 0.8 & 1.1 & 1.1 & 1.7 & 4.8 & 0.8 & 1.1 & 1.1 & 1.7 & 4.9 & 5.2 \\
\hline Subsidies & 38.4 & 18.4 & 17.6 & 17.6 & 3.6 & 4.5 & 4.3 & 5.2 & 24.1 & 5.0 & 6.2 & 5.9 & 7.1 & 23.4 & 24.2 \\
\hline Interest & 10.4 & 16.2 & 37.1 & 37.1 & 6.2 & 8.6 & 7.7 & 14.7 & 50.3 & 8.2 & 14.9 & 10.3 & 16.9 & 54.0 & 57.7 \\
\hline Social allowances and pensions & 206.2 & 239.9 & 247.2 & 247.2 & 58.2 & 62.3 & 62.1 & 64.6 & 267.6 & 63.0 & 67.4 & 67.2 & 69.9 & 267.4 & 287.9 \\
\hline $\begin{array}{l}\text { Of which: pensions } \\
\text { Orloris }\end{array}$ & 153.5 & 176.0 & 181.2 & 181.2 & 43.7 & 44.2 & 45.6 & 47.7 & 196.5 & 47.4 & 47.9 & 49.4 & 51.7 & 196.7 & 215.4 \\
\hline Goods and services & 133.1 & 150.7 & 153.3 & 153.3 & 28.4 & 38.7 & 37.8 & 48.5 & 165.4 & 30.7 & 41.7 & 40.7 & 52.3 & 170.9 & 186.4 \\
\hline Grants & 52.9 & 62.0 & 66.8 & 66.8 & 14.2 & 17.4 & 16.8 & 18.3 & 57.6 & 12.2 & 15.0 & 14.5 & 15.8 & 56.6 & 57.7 \\
\hline Other expenditure & 133.9 & 136.6 & 140.2 & 140.2 & 23.3 & 29.2 & 38.6 & 49.1 & 144.2 & 27.7 & 33.6 & 39.7 & 43.1 & 148.9 & 168.8 \\
\hline Transactions in nonfinancial assets & 141.5 & 185.1 & 168.9 & 170.0 & 36.7 & 42.2 & 54.6 & 35.4 & 199.6 & 42.6 & 60.3 & 60.5 & 36.2 & 194.7 & 205.7 \\
\hline Acquisition of nonfinancial assets & 162.6 & 195.7 & 169.0 & 170.0 & 36.8 & 42.7 & 54.6 & 34.9 & 199.6 & 42.6 & 60.3 & 60.5 & 36.2 & 194.7 & 205.7 \\
\hline Of which: projects rel & & 26.5 & 23.0 & 22.0 & 8.8 & 9.7 & 4.5 & 0.0 & 30.0 & 11.5 & 18.5 & 0.0 & 0.0 & 10.6 & 0.0 \\
\hline Disposals of nonfinancial assets & 21.1 & 10.6 & 0.0 & 0.0 & 0.1 & 0.5 & 0.0 & -0.5 & 0.0 & 0.0 & 0.0 & 0.0 & 0.0 & 0.0 & 0.0 \\
\hline Overall balance (above-the-line) & -62.7 & -241.5 & -167.9 & -163.1 & -30.9 & -26.5 & -46.3 & -64.2 & -147.8 & -31.6 & -35.3 & -38.0 & -42.9 & -118.0 & -105.2 \\
\hline Statistical discrepancy & 21.0 & -6.2 & 0.0 & 0.0 & 10.9 & -6.2 & -2.3 & -2.3 & 0.0 & 0.0 & 0.0 & 0.0 & 0.0 & 0.0 & 0.0 \\
\hline Overall balance (below-the-line) & -41.7 & -247.7 & -167.9 & -163.1 & -20.0 & -32.7 & -48.6 & -66.5 & -147.8 & -31.6 & -35.3 & -38.0 & -42.9 & -118.0 & -105.2 \\
\hline Financing & 41.7 & 247.7 & 167.9 & 163.1 & 20.0 & 32.7 & 48.6 & 66.5 & 147.8 & 31.6 & 35.3 & 38.0 & 42.9 & 118.0 & 105.2 \\
\hline Domestic financing & 23.7 & -108.5 & 93.7 & 66.1 & 7.7 & 10.9 & 33.1 & 42.0 & 63.5 & 16.5 & 14.5 & 19.1 & 13.4 & 70.3 & 115.7 \\
\hline Banking system 1/ & -9.6 & -40.0 & 99.4 & 70.2 & -6.9 & 16.2 & 42.4 & 47.8 & 54.5 & 16.0 & 13.8 & 15.4 & 9.3 & 63.3 & 98.3 \\
\hline 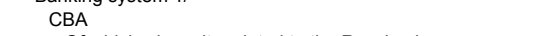 & -33.0 & -54.4 & 76.4 & 52.3 & 6.5 & 14.3 & 19.1 & 36.6 & 25.5 & 14.7 & 13.1 & 2.7 & -5.0 & 33.3 & 63.8 \\
\hline Of which: deposits related to the Russian loan & 0.0 & -76.6 & 38.5 & 37.5 & 4.4 & 12.5 & 16.3 & 5.2 & 27.1 & 10.8 & 17.8 & -0.7 & -0.7 & 7.7 & \\
\hline Commercial Banks & 23.4 & 14.4 & 23.0 & 17.9 & -13.4 & 1.9 & 23.3 & 11.2 & 29.0 & 1.3 & 0.8 & 12.7 & 14.3 & 30.0 & 34.5 \\
\hline Nonbanks & 33.4 & -68.5 & -5.8 & -4.2 & 14.6 & -5.3 & -9.3 & -5.8 & 9.0 & 0.6 & 0.7 & 3.7 & 4.1 & 7.0 & 17.4 \\
\hline Privatization proceeds & 31.6 & 0.0 & 0.0 & 0.0 & 0.0 & 0.0 & 0.0 & 0.0 & 0.0 & 0.0 & 0.0 & 0.0 & 0.0 & 0.0 & 0.0 \\
\hline T-Bills & 3.5 & 8.7 & 8.2 & 9.8 & 6.8 & -1.9 & 2.0 & 1.3 & 6.0 & -0.2 & -0.1 & 2.9 & 3.3 & 3.0 & 3.5 \\
\hline Promissory note/ther & $\begin{array}{c}.5 \\
-1.2\end{array}$ & $\begin{array}{l}. .1 \\
-3.2\end{array}$ & $\begin{array}{l}0.2 \\
-2.0\end{array}$ & $\begin{array}{r}-2.0 \\
-2.0\end{array}$ & $\begin{array}{l}0.0 \\
0.0\end{array}$ & 0.0 & 0.0 & $\begin{array}{l}1.0 \\
-2.0\end{array}$ & 0.0 & 0.0 & 0.0 & 0.0 & 0.0 & 0.0 & 0.0 \\
\hline Net lending & -0.5 & -74.0 & -11.9 & -12.0 & 7.8 & -3.3 & -11.3 & -5.1 & 3.0 & 0.8 & 0.8 & 0.8 & 0.8 & 4.0 & 13.9 \\
\hline Of which: financed with the Russian loan & & -78.8 & -13.5 & -13.5 & 4.4 & -2.8 & -11.8 & -3.3 & 2.9 & 0.7 & 0.7 & 0.7 & 0.7 & 2.9 & 13.1 \\
\hline External financing & 17.9 & 356.2 & 74.2 & 97.0 & 12.2 & 21.8 & 15.6 & 24.6 & 84.3 & 15.1 & 20.8 & 18.9 & 29.5 & 47.7 & -10.5 \\
\hline Gross inflov & 48.8 & 395.6 & 118.0 & 141.2 & 24.3 & 32.0 & 27.7 & 34.0 & 131.2 & 28.2 & 31.6 & 32.1 & 39.4 & 116.1 & 116.6 \\
\hline Russian project loan & & 185.1 & 0.0 & 0.0 & 0.0 & 0.0 & 0.0 & 0.0 & 0.0 & 0.0 & 0.0 & 0.0 & 0.0 & 0.0 & 0.0 \\
\hline Amortizati & -4.9 & -6.3 & -9.9 & -10.3 & -3.4 & -1.2 & -3.5 & -1.7 & -11.9 & -4.1 & -1.6 & -4.2 & -2.0 & -31.8 & -87.3 \\
\hline Net lending & -26.0 & $\begin{array}{r}-33.1 \\
-1\end{array}$ & -33.9 & -33.9 & -8.7 & -9.0 & -8.6 & -7.6 & -35.1 & -9.0 & -9.3 & -8.9 & -7.9 & -36.7 & -39.7 \\
\hline Memorandun & & & & & & & & & & & & & & & \\
\hline illion of drams) & 3,568 & 3,103 & 3,470 & 3,433 & 3,470 & 3,470 & 3,470 & 3,470 & 3,771 & 3,771 & 3,771 & 3,771 & 3,771 & 4,100 & 4,456 \\
\hline Program balance $3 /$ & -68.2 & -354.8 & -140.4 & -132.6 & 3.5 & -27.9 & -51.8 & -64.2 & -112.3 & -33.7 & -24.1 & -31.9 & -22.6 & -34.6 & -14.5 \\
\hline Overall balance excl. spending financed with the Russian loan & $\ldots$ & -215.0 & -144.9 & -141.1 & -22.0 & -16.8 & -41.8 & -64.2 & -117.8 & -20.1 & -16.8 & -38.0 & -42.9 & -107.4 & -105.2 \\
\hline Budget support & & 290.9 & 44.7 & 64.9 & 0.0 & 14.8 & 11.0 & 18.8 & 63.6 & 22.0 & 11.9 & 17.8 & 11.9 & 0.0 & 0.0 \\
\hline T-bill issuance & 25.3 & 45.4 & 30.3 & 30.0 & 0.6 & -3.0 & 20.1 & 12.6 & 30.0 & -0.9 & -0.3 & 14.6 & 16.6 & 30.0 & 35.0 \\
\hline
\end{tabular}

Sources: Ministry of Finance and Economy, Central Bank of Armenia, and Fund staff estimates and projections.

1/ Discrepancy between the fiscal and monetary accounts in 2009Q3-Q4 and 2010 is explained by government lending to the economy through commercial banks.

3/ The program balance until 2009 is measured as below-the-line overall balance minus net lending. From 2010, it is measured as in 2009 less project financing. 
Table 6. Armenia: Central Government Operations, 2008-13

(In percent of GDP, unless otherwise specified)

\begin{tabular}{|c|c|c|c|c|c|c|c|c|c|c|c|c|c|c|c|}
\hline & \multirow{3}{*}{$\begin{array}{r}2008 \\
\text { Act. }\end{array}$} & \multirow[t]{2}{*}{2009} & \multicolumn{2}{|c|}{2010} & \multicolumn{4}{|c|}{2010} & \multicolumn{5}{|c|}{2011} & \multirow[t]{2}{*}{2012} & \multirow[t]{2}{*}{2013} \\
\hline & & & \multirow{2}{*}{\multicolumn{2}{|c|}{ Country Rep. }} & Q1 & Q2 & Q3 & Q4 & & Q1 & Q2 & Q3 & Q4 & & \\
\hline & & Prel. & Proj. & & Prel. & Prel. & Proj. & Proj. & Proj. & Proj. & Proj. & Proj. & Proj. & Proj. & Proj. \\
\hline Total revenue and grants & 20.5 & 21.1 & 21.8 & 22.2 & 4.6 & 5.7 & 5.8 & 5.8 & 22.7 & 4.7 & 6.0 & 6.0 & 6.0 & 21.8 & 22.2 \\
\hline Total revenue & 20.1 & 20.4 & 20.5 & 20.6 & 4.4 & 5.5 & 5.4 & 5.2 & 21.0 & 4.5 & 5.6 & 5.5 & 5.3 & 21.4 & 21.8 \\
\hline Tax revenues & 16.8 & 16.3 & 16.8 & 16.8 & 3.5 & 4.5 & 4.5 & 4.4 & 17.2 & 3.6 & 4.6 & 4.6 & 4.5 & 17.6 & 18.0 \\
\hline VAT & 8.3 & 7.7 & 7.9 & 7.9 & 1.7 & 2.2 & 2.2 & 1.9 & 8.0 & 1.7 & 2.2 & 2.3 & 1.9 & 8.2 & 8.3 \\
\hline Profits, simplified and presumptive & 3.2 & 3.4 & 3.2 & 3.2 & 0.7 & 0.9 & 0.8 & 0.9 & 3.3 & 0.7 & 0.9 & 0.8 & 0.9 & 3.3 & 3.4 \\
\hline Personal income tax & 1.5 & 1.9 & 2.0 & 2.0 & 0.5 & 0.5 & 0.5 & 0.5 & 2.1 & 0.5 & 0.5 & 0.5 & 0.5 & 2.1 & 2.1 \\
\hline Customs duties & 1.0 & 0.8 & 1.0 & 1.0 & 0.2 & 0.2 & 0.2 & 0.3 & 1.2 & 0.2 & 0.3 & 0.3 & 0.4 & 1.2 & 1.2 \\
\hline Other & 2.7 & 2.5 & 2.7 & 2.7 & 0.5 & 0.6 & 0.7 & 0.8 & 2.8 & 0.5 & 0.7 & 0.8 & 0.8 & 2.8 & 3.0 \\
\hline Social contributions & 2.9 & 3.3 & 3.0 & 3.1 & 0.8 & 0.8 & 0.8 & 0.7 & 3.2 & 0.8 & 0.9 & 0.8 & 0.7 & 3.2 & 3.3 \\
\hline Other revenue & 0.4 & 0.8 & 0.7 & 0.7 & 0.2 & 0.3 & 0.1 & 0.1 & 0.6 & 0.1 & 0.2 & 0.1 & 0.1 & 0.5 & 0.5 \\
\hline Grants & 0.4 & 0.7 & 1.2 & 1.6 & 0.1 & 0.2 & 0.4 & 0.5 & 1.7 & 0.2 & 0.3 & 0.5 & 0.7 & 0.4 & 0.4 \\
\hline Total expenditure & 22.2 & 28.9 & 26.6 & 26.9 & 5.4 & 6.5 & 7.1 & 7.6 & 26.6 & 5.5 & 6.9 & 7.0 & 7.2 & 24.7 & 24.6 \\
\hline Expense & 18.3 & 22.9 & 21.7 & 22.0 & 4.4 & 5.2 & 5.5 & 6.6 & 21.3 & 4.4 & 5.3 & 5.4 & 6.2 & 20.0 & 20.0 \\
\hline Wages & 2.0 & 2.7 & 2.5 & 2.5 & 0.5 & 0.6 & 0.7 & 0.8 & 2.4 & 0.5 & 0.6 & 0.6 & 0.7 & 2.2 & 2.3 \\
\hline Pensions & 0.1 & 0.1 & 0.1 & 0.1 & 0.0 & 0.0 & 0.0 & 0.0 & 0.1 & 0.0 & 0.0 & 0.0 & 0.0 & 0.1 & 0.1 \\
\hline Subsidies & 1.1 & 0.6 & 0.5 & 0.5 & 0.1 & 0.1 & 0.1 & 0.1 & 0.6 & 0.1 & 0.2 & 0.2 & 0.2 & 0.6 & 0.5 \\
\hline Interest & 0.3 & 0.5 & 1.1 & 1.1 & 0.2 & 0.2 & 0.2 & 0.4 & 1.3 & 0.2 & 0.4 & 0.3 & 0.4 & 1.3 & 1.3 \\
\hline Social allowances and pensions & 5.8 & 7.7 & 7.1 & 7.2 & 1.7 & 1.8 & 1.8 & 1.9 & 7.1 & 1.7 & 1.8 & 1.8 & 1.9 & 6.5 & 6.5 \\
\hline Of which: pensions & 4.3 & 5.7 & 5.2 & 5.3 & 1.3 & 1.3 & 1.3 & 1.4 & 5.2 & 1.3 & 1.3 & 1.3 & 1.4 & 4.8 & 4.8 \\
\hline Goods and services & 3.7 & 4.9 & 4.4 & 4.5 & 0.8 & 1.1 & 1.1 & 1.4 & 4.4 & 0.8 & 1.1 & 1.1 & 1.4 & 4.2 & 4.2 \\
\hline Grants & 1.5 & 2.0 & 1.9 & 1.9 & 0.4 & 0.5 & 0.5 & 0.5 & 1.5 & 0.3 & 0.4 & 0.4 & 0.4 & 1.4 & 1.3 \\
\hline Other expenditure & 3.8 & 4.4 & 4.0 & 4.1 & 0.7 & 0.8 & 1.1 & 1.4 & 3.8 & 0.7 & 0.9 & 1.1 & 1.1 & 3.6 & 3.8 \\
\hline Transactions in nonfinancial assets & 4.0 & 6.0 & 4.9 & 5.0 & 1.1 & 1.2 & 1.6 & 1.0 & 5.3 & 1.1 & 1.6 & 1.6 & 1.0 & 4.7 & 4.6 \\
\hline Acquisition of nonfinancial assets & 4.6 & 6.3 & 4.9 & 5.0 & 1.1 & 1.2 & 1.6 & 1.0 & 5.3 & 1.1 & 1.6 & 1.6 & 1.0 & 4.7 & 4.6 \\
\hline Of which: projects related to the Russian loan & & 0.9 & 0.7 & 0.6 & 0.3 & 0.3 & 0.1 & 0.0 & 0.8 & 0.3 & 0.5 & 0.0 & 0.0 & 0.3 & 0.0 \\
\hline Disposals of nonfinancial assets & 0.6 & 0.3 & 0.0 & 0.0 & 0.0 & 0.0 & 0.0 & 0.0 & 0.0 & 0.0 & 0.0 & 0.0 & 0.0 & 0.0 & 0.0 \\
\hline Overall balance (above-the-line) & -1.8 & -7.8 & -4.8 & -4.8 & -0.9 & -0.8 & -1.3 & -1.9 & -3.9 & -0.8 & -0.9 & -1.0 & -1.1 & -2.9 & -2.4 \\
\hline Statistical discrepancy & 0.6 & -0.2 & 0.0 & 0.0 & 0.3 & -0.2 & -0.1 & -0.1 & 0.0 & 0.0 & 0.0 & 0.0 & 0.0 & 0.0 & 0.0 \\
\hline Overall balance (below-the-line) & -1.2 & -8.0 & -4.8 & -4.8 & -0.6 & -0.9 & -1.4 & -1.9 & -3.9 & -0.8 & -0.9 & -1.0 & -1.1 & -2.9 & -2.4 \\
\hline Financing & 1.2 & 8.0 & 4.8 & 4.8 & 0.6 & 0.9 & 1.4 & 1.9 & 3.9 & 0.8 & 0.9 & 1.0 & 1.1 & 2.9 & 2.4 \\
\hline Domestic financing & 0.7 & -3.5 & 2.7 & 1.9 & 0.2 & 0.3 & 1.0 & 1.2 & 1.7 & 0.4 & 0.4 & 0.5 & 0.4 & 1.7 & 2.6 \\
\hline Banking system 1/ & -0.3 & -1.3 & 2.9 & 2.0 & -0.2 & 0.5 & 1.2 & 1.4 & 1.4 & 0.4 & 0.4 & 0.4 & 0.2 & 1.5 & 2.2 \\
\hline CBA & -0.9 & -1.8 & 2.2 & 1.5 & 0.2 & 0.4 & 0.5 & 1.1 & 0.7 & 0.4 & 0.3 & 0.1 & -0.1 & 0.8 & 1.4 \\
\hline Of which: deposits related to the Russian loan & & -2.5 & 1.1 & 1.1 & 0.1 & 0.4 & 0.5 & 0.2 & 0.7 & 0.3 & 0.5 & 0.0 & 0.0 & 0.2 & \\
\hline Commercial Banks & 0.7 & 0.5 & 0.7 & 0.5 & -0.4 & 0.1 & 0.7 & 0.3 & 0.8 & 0.0 & 0.0 & 0.3 & 0.4 & 0.7 & 0.8 \\
\hline Nonbanks & 0.9 & -2.2 & -0.2 & -0.1 & 0.4 & -0.2 & -0.3 & -0.2 & 0.2 & 0.0 & 0.0 & 0.1 & 0.1 & 0.2 & 0.4 \\
\hline Privatization proceeds & 0.9 & 0.0 & 0.0 & 0.0 & 0.0 & 0.0 & 0.0 & 0.0 & 0.0 & 0.0 & 0.0 & 0.0 & 0.0 & 0.0 & 0.0 \\
\hline T-Bills & 0.1 & 0.3 & 0.2 & 0.3 & 0.2 & -0.1 & 0.1 & 0.0 & 0.2 & 0.0 & 0.0 & 0.1 & 0.1 & 0.1 & 0.1 \\
\hline Promissory note/other & 0.0 & -0.1 & -0.1 & -0.1 & 0.0 & 0.0 & 0.0 & -0.1 & 0.0 & 0.0 & 0.0 & 0.0 & 0.0 & 0.0 & 0.0 \\
\hline Net lending & 0.0 & -2.4 & -0.3 & -0.3 & 0.2 & -0.1 & -0.3 & -0.1 & 0.1 & 0.0 & 0.0 & 0.0 & 0.0 & 0.1 & 0.3 \\
\hline Of which: financed with the Russian loan & $\ldots$ & -2.5 & -0.4 & -0.4 & 0.1 & -0.1 & -0.3 & -0.1 & 0.1 & 0.0 & 0.0 & 0.0 & 0.0 & 0.1 & 0.3 \\
\hline External financing & 0.5 & 11.5 & 2.1 & 2.8 & 0.4 & 0.6 & 0.4 & 0.7 & 2.2 & 0.4 & 0.6 & 0.5 & 0.8 & 1.2 & -0.2 \\
\hline Gross inflow 21 & 1.4 & 12.8 & 3.4 & 4.1 & 0.7 & 0.9 & 0.8 & 1.0 & 3.5 & 0.7 & 0.8 & 0.9 & 1.0 & 2.8 & 2.6 \\
\hline Of which: Russian project loan & & 6.0 & 0.0 & 0.0 & 0.0 & 0.0 & 0.0 & 0.0 & 0.0 & 0.0 & 0.0 & 0.0 & 0.0 & 0.0 & 0.0 \\
\hline Amortization due & -0.1 & -0.2 & -0.3 & -0.3 & -0.1 & 0.0 & -0.1 & -0.1 & -0.3 & -0.1 & 0.0 & -0.1 & -0.1 & -0.8 & -2.0 \\
\hline Net lending & -0.7 & -1.1 & -1.0 & -1.0 & -0.2 & -0.3 & -0.2 & -0.2 & -0.9 & -0.2 & -0.2 & -0.2 & -0.2 & -0.9 & -0.9 \\
\hline Memorandum items: & & & & & & & & & & & & & & & \\
\hline Nominal GDP (in billion of drams) & 3,568 & 3,103 & 3,470 & 3,433 & 3,470 & 3,470 & 3,470 & 3,470 & 3,771 & 3,771 & 3,771 & 3,771 & 3,771 & 4,100 & 4,456 \\
\hline Program balance $3 /$ & -1.9 & -11.4 & -4.0 & -3.9 & 0.1 & -0.8 & -1.5 & -1.8 & -3.0 & -0.9 & -0.6 & -0.8 & -0.6 & -0.8 & -0.4 \\
\hline Overall balance excl. spending financed with the Russian loan & $\ldots$ & -6.9 & -4.2 & -4.1 & -0.6 & -0.5 & -1.2 & -1.9 & -3.1 & -0.5 & -0.4 & -1.0 & -1.1 & -2.6 & -2.6 \\
\hline Budget support & $\ldots$ & 9.4 & 1.3 & 1.9 & 0.0 & 0.4 & 0.3 & 0.5 & 1.7 & 0.6 & 0.3 & 0.5 & 0.3 & 0.0 & 0.0 \\
\hline T-bill issuance & 0.7 & 1.5 & 0.9 & 0.9 & 0.0 & -0.1 & 0.6 & 0.4 & 0.8 & 0.0 & 0.0 & 0.4 & 0.4 & 0.7 & 0.9 \\
\hline
\end{tabular}

Sources: Ministry of Finance and Economy, Central Bank of Armenia, and Fund staff estimates and projections.

1/ Discrepancy between the fiscal and monetary accounts in 2009Q3-Q4 and 2010 is explained by government lending to the economy through commercial banks.

$3 /$ The program balance until 2009 
Table 7. Armenia: Medium-Term Macroeconomic Framework, 2007-15

(In percent of GDP, unless otherwise specified)

\begin{tabular}{|c|c|c|c|c|c|c|c|c|c|}
\hline & 2007 & 2008 & 2009 & 2010 & 2011 & 2012 & 2013 & 2014 & 2015 \\
\hline & Act. & Act. & Prel. & \multicolumn{6}{|c|}{ Projections } \\
\hline \multicolumn{10}{|l|}{ National income and prices } \\
\hline Real GDP (percent change) & 13.7 & 6.9 & -14.2 & 4.0 & 4.6 & 4.3 & 4.2 & 4.0 & 4.0 \\
\hline Gross domestic product (in millions of U.S. dollars) & 9,206 & 11,662 & 8,541 & 8,836 & 8,858 & 9,199 & 9,711 & 10,317 & 10,960 \\
\hline Gross national income per capita (in U.S. dollars) & 2,940 & 3,752 & 2,666 & 2,750 & 2,741 & 2,828 & 2,965 & 3,128 & 3,287 \\
\hline $\mathrm{CPI}$ inflation, end of period (percent change) & 6.6 & 5.2 & 6.6 & 7.1 & 4.6 & 4.0 & 4.0 & 4.0 & 4.0 \\
\hline \multicolumn{10}{|l|}{ Investment and saving } \\
\hline Private & 32.9 & 39.8 & 28.0 & 28.5 & 29.0 & 29.5 & 30.0 & 30.5 & 31.0 \\
\hline Public & 5.2 & 4.0 & 6.0 & 4.9 & 5.3 & 4.7 & 4.6 & 4.6 & 4.6 \\
\hline National savings & 31.8 & 32.0 & 17.9 & 18.7 & 21.7 & 22.6 & 24.4 & 26.7 & 27.4 \\
\hline Private & 28.9 & 29.8 & 19.7 & 18.7 & 20.3 & 20.7 & 22.2 & 24.3 & 24.8 \\
\hline Public & 2.9 & 2.2 & -1.8 & 0.0 & 1.4 & 1.9 & 2.3 & 2.4 & 2.7 \\
\hline \multicolumn{10}{|l|}{ Central government operations } \\
\hline Revenue and grants & 20.1 & 20.5 & 21.1 & 21.8 & 22.7 & 21.8 & 22.2 & 22.4 & 22.6 \\
\hline Of which: tax revenue & 16.0 & 16.8 & 16.3 & 16.8 & 17.2 & 17.6 & 18.0 & 18.3 & 18.6 \\
\hline Expenditure & 22.4 & 22.2 & 28.9 & 26.6 & 26.6 & 24.7 & 24.6 & 24.6 & 24.6 \\
\hline Current expenditure & 17.2 & 18.3 & 22.9 & 21.7 & 21.3 & 20.0 & 20.0 & 20.0 & 20.0 \\
\hline Capital expenditure & 5.2 & 4.0 & 6.0 & 4.9 & 5.3 & 4.7 & 4.6 & 4.6 & 4.6 \\
\hline Overall balance on a cash basis & -2.2 & -1.2 & -8.0 & -4.8 & -3.9 & -2.9 & -2.4 & -2.2 & -2.0 \\
\hline Domestic financing & 1.0 & 0.7 & -3.5 & 2.7 & 1.7 & 1.7 & 2.6 & 3.3 & 2.9 \\
\hline External financing & 1.2 & 0.5 & 11.5 & 2.1 & 2.2 & 1.2 & -0.2 & -1.1 & -0.9 \\
\hline Government and government-guaranteed debt & 16.1 & 16.2 & 40.6 & 44.1 & 48.9 & 46.5 & 42.9 & 38.4 & 36.2 \\
\hline \multicolumn{10}{|l|}{ External sector } \\
\hline Exports of goods and services & 19.3 & 15.1 & 15.6 & 17.1 & 19.4 & 20.9 & 21.9 & 22.7 & 23.1 \\
\hline Imports of goods and services & 39.0 & 40.7 & 43.1 & 44.0 & 46.6 & 47.6 & 47.9 & 47.7 & 47.6 \\
\hline Current account (in percent of GDP) & -6.4 & -11.8 & -16.0 & -14.6 & -12.6 & -11.7 & -10.1 & -8.4 & -8.1 \\
\hline Current account (in millions of U.S. dollars) & -589 & $-1,382$ & $-1,367$ & $-1,289$ & $-1,112$ & $-1,072$ & -985 & -864 & -892 \\
\hline Capital and financial account (in millions of U.S. dollars) & 1,191 & 1,134 & 1,500 & 855 & 967 & 1,059 & 1,019 & 1,071 & 1,069 \\
\hline Of which: direct foreign investment & 701 & 940 & 725 & 761 & 799 & 839 & 881 & 925 & 962 \\
\hline public sector disbursements & 149 & 160 & 907 & 289 & 308 & 261 & 254 & 250 & 106 \\
\hline Change in gross international reserves (in millions of U.S. dollars) $1 /$ & -587 & 252 & -597 & 264 & 49 & 60 & 156 & -53 & -160 \\
\hline Of which: IMF & 0 & 0 & 0 & 54 & 109 & 101 & 83 & 0 & 0 \\
\hline Other & 0 & 0 & 0 & 0 & 10 & 14 & 15 & 0 & 0 \\
\hline Gross international reserves in months of imports & 4.2 & 4.6 & 6.2 & 5.1 & 4.9 & 4.5 & 3.8 & 3.7 & 3.9 \\
\hline
\end{tabular}

Sources: Armenian authorities; and Fund staff estimates and projections.

1/ A negative figure indicates an increase. 
Table 8. Armenia: Proposed Fund Disbursments and Timing of Reviews under a Three-year EFF/ECF Blend

\begin{tabular}{|c|c|c|c|c|c|c|c|}
\hline \multirow[t]{2}{*}{ Date of Availability } & \multirow[t]{2}{*}{ Conditions } & \multicolumn{3}{|c|}{ Amount (millions of SDRs) } & \multicolumn{3}{|c|}{ Percent of Quota } \\
\hline & & ECF & EFF & Total & ECF & EFF & Total \\
\hline June 28, 2010 & Board approval of the arrangement & 18.70 & 17.50 & 36.20 & 20.33 & 19.02 & 39.35 \\
\hline September 30, 2010 & Observance of end-June 2010 performance criteria and completion of first review & 18.70 & 17.50 & 36.20 & 20.33 & 19.02 & 39.35 \\
\hline March 30, 2011 & Observance of end-December 2010 performance criteria and completion of second review & 18.70 & 17.50 & 36.20 & 20.33 & 19.02 & 39.35 \\
\hline September 30, 2011 & Observance of end-June 2011 performance criteria and completion of third review & 18.70 & 17.50 & 36.20 & 20.33 & 19.02 & 39.35 \\
\hline March 30, 2012 & Observance of end-December 2011 performance criteria and completion of fourth review & 16.00 & 17.50 & 33.50 & 17.39 & 19.02 & 36.41 \\
\hline September 30, 2012 & Observance of end-June 2012 performance criteria and completion of fifth review & 16.00 & 17.50 & 33.50 & 17.39 & 19.02 & 36.41 \\
\hline \multirow[t]{2}{*}{ March 30, 2013} & Observance of end-December 2012 performance criteria and completion of sixth review & 26.60 & 28.40 & 55.00 & 28.91 & 30.87 & 59.78 \\
\hline & Total & 133.40 & 133.40 & 266.80 & 145.00 & 145.00 & 290.00 \\
\hline
\end{tabular}

Source: Fund staff estimates and projections. 
Table 9. Armenia: Indicators of Capacity to Repay the Fund, 2010-18 1/

\begin{tabular}{|c|c|c|c|c|c|c|c|c|c|}
\hline & 2010 & 2011 & 2012 & 2013 & 2014 & 2015 & 2016 & 2017 & 2018 \\
\hline & \multicolumn{9}{|c|}{ Projections } \\
\hline \multicolumn{10}{|l|}{$\begin{array}{l}\text { Fund obligations based on existing credit } \\
\text { (in millions of SDRs) }\end{array}$} \\
\hline Principal & 7.2 & 14.4 & 101.0 & 180.2 & 98.1 & 13.5 & 9.9 & 8.6 & 7.3 \\
\hline Charges and interest & 1.3 & 5.4 & 5.3 & 3.7 & 1.4 & 0.6 & 0.5 & 0.4 & 0.4 \\
\hline \multicolumn{10}{|l|}{$\begin{array}{l}\text { Fund obligations based on existing and prospective credit } \\
\text { (in millions of SDRs) }\end{array}$} \\
\hline Principal & 7.2 & 14.4 & 101.0 & 180.2 & 98.1 & 17.9 & 25.7 & 38.3 & 46.9 \\
\hline Charges and interest & 1.8 & 8.4 & 9.5 & 7.5 & 3.3 & 2.5 & 2.3 & 2.0 & 1.7 \\
\hline \multicolumn{10}{|l|}{ Total obligations based on existing and prospective credit } \\
\hline In millions of SDRs & 9.1 & 22.8 & 110.5 & 187.7 & 101.4 & 20.4 & 28.0 & 40.4 & 48.5 \\
\hline In millions of U.S. dollars & 14.5 & 36.3 & 176.0 & 299.1 & 161.4 & 32.2 & 44.2 & 63.8 & 76.7 \\
\hline In percent of gross international reserves & 0.8 & 2.0 & 10.2 & 19.0 & 9.9 & 1.8 & 2.3 & 3.0 & 3.4 \\
\hline In percent of exports of goods and services & 1.0 & 2.1 & 9.1 & 14.1 & 6.9 & 1.3 & 1.6 & 2.1 & 2.4 \\
\hline In percent of debt service $2 /$ & 12.5 & 27.6 & 63.2 & 67.1 & 52.6 & 14.6 & 17.9 & 22.8 & 26.0 \\
\hline In percent of GDP & 0.2 & 0.4 & 1.9 & 3.1 & 1.6 & 0.3 & 0.4 & 0.5 & 0.6 \\
\hline In percent of quota & 9.9 & 24.8 & 120.1 & 204.1 & 110.2 & 22.1 & 30.4 & 43.9 & 52.7 \\
\hline \multicolumn{10}{|l|}{ Outstanding Fund credit 2/ } \\
\hline In millions of SDRs & 481.0 & 539.0 & 505.0 & 379.7 & 281.7 & 263.8 & 238.1 & 199.7 & 152.9 \\
\hline In billions of U.S. dollars & 0.8 & 0.9 & 0.8 & 0.6 & 0.4 & 0.4 & 0.4 & 0.3 & 0.2 \\
\hline In percent of gross international reserves & 44.1 & 48.0 & 46.5 & 38.4 & 27.6 & 23.3 & 19.4 & 15.1 & 10.6 \\
\hline In percent of exports of goods and services & 50.7 & 49.9 & 41.8 & 28.5 & 19.1 & 16.4 & 13.7 & 10.6 & 7.5 \\
\hline In percent of debt service 2/ & 660.8 & 653.0 & 289.1 & 135.8 & 146.2 & 189.8 & 152.6 & 112.8 & 81.9 \\
\hline In percent of GDP & 8.7 & 9.7 & 8.7 & 6.2 & 4.3 & 3.8 & 3.2 & 2.6 & 1.8 \\
\hline In percent of quota & 522.8 & 585.8 & 548.9 & 412.8 & 306.2 & 286.7 & 258.8 & 217.1 & 166.2 \\
\hline Net use of Fund credit (in millions of SDRs) & 113.7 & 58.0 & -34.0 & -125.2 & -98.1 & -17.9 & -25.7 & -38.3 & -46.9 \\
\hline Disbursements & 120.9 & 72.4 & 67.0 & 55.0 & 0.0 & 0.0 & 0.0 & 0.0 & 0.0 \\
\hline Repayments and repurchases & 7.2 & 14.4 & 101.0 & 180.2 & 98.1 & 17.9 & 25.7 & 38.3 & 46.9 \\
\hline \multicolumn{10}{|l|}{ Memorandum items: } \\
\hline Nominal GDP (in millions of U.S. dollars) & $8,835.6$ & $8,858.2$ & $9,198.8$ & $9,711.2$ & $10,316.6$ & $10,959.8$ & $11,643.0$ & $12,368.8$ & $13,139.9$ \\
\hline Exports of goods and services (in millions of U.S. dollars) & $1,513.4$ & $1,720.2$ & $1,926.6$ & $2,123.9$ & $2,342.2$ & $2,537.0$ & $2,748.8$ & $2,979.3$ & $3,230.2$ \\
\hline Gross international reserves (in millions of U.S. dollars) & $1,740.1$ & $1,789.5$ & $1,730.0$ & $1,573.9$ & $1,626.9$ & $1,787.0$ & $1,940.2$ & $2,094.4$ & $2,270.8$ \\
\hline Debt service (in millions of U.S. dollars) $2 /$ & 116.0 & 131.4 & 278.3 & 445.6 & 306.8 & 219.8 & 246.7 & 280.0 & 295.2 \\
\hline Quota (in millions of SDRs) & 92.0 & 92.0 & 92.0 & 92.0 & 92.0 & 92.0 & 92.0 & 92.0 & 92.0 \\
\hline
\end{tabular}

Source: Fund staff estimates and projections.

1/ Indicators cover both GRA and ECF credit.

2/ Total debt service includes IMF obligations. 
Table 10. Armenia: Structural Benchmarks for Future Implementation Under the EFF/ECF

\begin{tabular}{|c|c|c|}
\hline Item & Measure & Desired Outcome \\
\hline \multicolumn{3}{|c|}{ Tax administration } \\
\hline 1 & $\begin{array}{l}\text { Develop manuals in tourism, real estate, transport, and sectors using cash register machines for tax audits for usage starting January } 2011 . \\
\text { (end-December 2010, modified) }\end{array}$ & \multirow{4}{*}{$\begin{array}{l}\text { Reduce corruption, increase tax } \\
\text { compliance, and enhance } \\
\text { revenue collection }\end{array}$} \\
\hline 2 & $\begin{array}{l}\text { Simplify the reporting system by reducing the frequency of reporting to tax authorities and considerably streamline tax forms for VAT, profits } \\
\text { tax, and personal income tax. (end-December 2010) }\end{array}$ & \\
\hline 3 & $\begin{array}{l}\text { Adopt a government decree establishing a mechanism for implementing a fully functional risk management approach in VAT refund } \\
\text { processing. (end-December 2010) }\end{array}$ & \\
\hline 4 & Implement a fully functional risk-based management approach in VAT refund processing. (end-June 2011, new) & \\
\hline \multicolumn{3}{|c|}{ Social policy } \\
\hline 5 & $\begin{array}{l}\text { Submit amendments to existing laws to parliament to enable the full functioning of an integrated system for the provision of social protection } \\
\text { services. (end-December 2011, modified) }\end{array}$ & Reduce poverty \\
\hline \multicolumn{3}{|c|}{ Financial sector } \\
\hline 6 & Issue prudential regulation requiring banks to prepare their contingency plans. (end-December 2010) & $\begin{array}{l}\text { Increase the stability of the } \\
\text { financial sector }\end{array}$ \\
\hline
\end{tabular}


Table 11. Armenia: Millennium Development Goals, 1993-2010

\begin{tabular}{|c|c|c|c|c|c|c|c|c|c|c|}
\hline & & 1993 & 1996 & 2000 & 2005 & 2006 & 2007 & 2008 & 2009 & 2010 \\
\hline \multicolumn{11}{|l|}{ Goal 1: Eradicate extreme poverty and hunger } \\
\hline Population below $\$ 1$ (PPP) per day, percentage & & & 17.5 & & & & 3.7 & & & \\
\hline Poverty gap ratio at $\$ 1$ a day (PPP), percentage & & & 4.7 & & & & 0.7 & & & \\
\hline Employment-to-population ratio, both sexes, percentage & & 36.1 & 37.7 & 37.4 & 38.6 & 38.4 & 39 & 38.1 & & \\
\hline Children under 5 moderately or severely underweight, percentage & & & & 2.6 & 4 & & & & & \\
\hline \multicolumn{11}{|l|}{ Goal 2: Achieve universal primary education } \\
\hline Primary completion rate, both sexes & & & & & 93.4 & 93 & 97.9 & & & \\
\hline Literacy rates of $15-24$ years old, both sexes, percentage & & & & & & & & 99.8 & & \\
\hline \multicolumn{11}{|l|}{ Goal 3: Promote gender equality and empower women } \\
\hline Gender Parity Index in primary level enrolment & & & & & 1.04 & 1.04 & 1.03 & 1.02 & & \\
\hline Share of women in wage employment in the non-agricultural sector & & & 51.8 & 52.1 & 44.1 & 45.7 & 44.8 & & & \\
\hline Seats held by women in national parliament, percentage & 35.6 & & & 3.1 & 5.3 & 5.3 & 5.3 & 9.2 & 8.4 & 9.2 \\
\hline \multicolumn{11}{|l|}{ Goal 4: Reduce child mortality } \\
\hline Children under five mortality rate per 1,000 live births & 56 & & & 36 & 27 & & & 23 & & \\
\hline \multicolumn{11}{|l|}{ Goal 5: Improve maternal health } \\
\hline Maternal mortality ratio per 100,000 live births & & & & & 76 & & & & & \\
\hline Births attended by skilled health personnel, percentage & & & & 96.8 & 97.8 & & 99.9 & & & \\
\hline Adolescent birth rate, per 1,000 women & 74.6 & 89.3 & 63.5 & 32.8 & 26.8 & 25.7 & 26.4 & & & \\
\hline \multicolumn{11}{|l|}{ Goal 6: Combat HIVIAIDS, malaria, and other diseases } \\
\hline People living with HIV, 15-49 years old, percentage & & & & & & & 0.1 & & & \\
\hline Antiretroviral therapy coverage among people with advanced $\mathrm{HIV}$ infection, percentage & & & & & & 8 & 12 & & & \\
\hline Tuberculosis prevalence rate per 100,000 population (mid-point) & 47 & 65 & 67 & 85 & 46 & 46 & 63 & 67 & & \\
\hline Tuberculosis detection rate under DOTS, percentage (mid-point) & & & 64 & 93 & 86 & 86 & 73 & 71 & & \\
\hline Tuberculosis treatment success rate under DOTS, percentage & & & 77 & 87 & 72 & 69 & 70 & & & \\
\hline \multicolumn{11}{|l|}{ Goal 7: Ensure environmental sustainability } \\
\hline Proportion of land area covered by forest, percentage & 12.3 & & & 10.8 & 10 & & & & & \\
\hline Carbon dioxide emissions (CO2), metric tons of $\mathrm{CO} 2$ per capita (CDIAC) & & 0.87 & 0.82 & 1.13 & 1.42 & 1.43 & 1.65 & & & \\
\hline Carbon dioxide emissions (CO2), kg CO2 per \$1 GDP (PPP) (CDIAC) & & 0.60 & 0.45 & 0.49 & 0.35 & 0.31 & 0.31 & & & \\
\hline Energy use (kg oil equivalent) per $\$ 1,000$ GDP (Constant 2005 PPP $\$$ ) & 740 & 447 & 304 & 284 & 199 & 179 & 176 & & & \\
\hline Terrestrial and marine areas protected to total territorial area, percentage & 6.93 & 6.93 & 6.93 & 6.93 & 7.99 & 7.99 & 7.99 & 7.99 & 7.99 & \\
\hline Proportion of the population using improved drinking water sources, total & & & & 93 & 95 & & & 96 & & \\
\hline Debt service as percentage of exports of goods and services and net income & & 1.1 & 9.6 & 7.9 & 3.2 & 2.7 & 2 & 1.9 & & \\
\hline Telephone lines per 100 population & 15.8 & 17.31 & 18.27 & 17.34 & 19.39 & 19.68 & 20.33 & 20.34 & & \\
\hline Mobile cellular telephone subscriptions per 100 population & 0 & 0 & 0.01 & 0.57 & 10.38 & 41.05 & 61.07 & 99.99 & & \\
\hline Internet users per 100 population & 0 & & 0.09 & 1.3 & 5.25 & 5.63 & 6.02 & 6.21 & & \\
\hline ODA received in landlocked developing countries as percentage of their GNI & & 9.95 & 17.8 & 10.99 & 3.38 & 3.27 & 3.69 & 2.44 & & \\
\hline
\end{tabular}

Source: http://mdgs.un.org/unsd/mdg/Data.aspx database on MDGs (UNSD_MDG_2010 Global Monitoring Data).

Goal 1. Halve, between 1990 and 2015, the proportion of people whose income is less than one dollar a day. Achieve full and productive employment and decent work for all, including women and young people. Halve, between 1990 and 2015, the proportion of people who suffer from hunger.

Goal 2. Ensure that, by 2015 , children everywhere, boys and girls alike, will be able to complete a full course of primary schooling

Goal 2. Ensure that, by 2015 , children everywhere, boys and girls alike, will be able to complete a full course of primary schooling.

Goal 4 . Reduce by two-thirds, between 1990 and 2015 , the under-five mortality rate.

Goal 5. Reduce by three quarters, between 1990 and 2015, the maternal mortality ratio.Achieve, by 2015, universal access to reproductive health

Goal 6. Have halted by 2015 and begun to reverse the spread of HIVIAIDS. Achieve, by 2010, universal access to treatment for HIV/AIDS for all those who need it. Have halted by 2015 and begun to reverse the incidence of malaria and other major diseases.

Goal 7. Integrate the principles of sustainable development into country policies and programmes and reverse the loss of environmental resources.Reduce biodiversity loss, achieving, by 2010 , a significant reduction in the rate of loss. Halve, by 2015 , the proportion of people without sustainable access to safe drinking water and basic sanitation. By 2020 , to have achieved a significant improvement in the lives of at least 100 million slum dwellers.

Goal 8. Develop further an open, rule-based, predictable, non-discriminatory trading and financial system. Includes a commitment to good governance, development and poverty reduction - both nationally and internationally. Address the special needs of the least developed countries

Includes: tariff and quota free access for the least developed countries' exports; enhanced programme of debt relief for heavily indebted poor countries (HIPC) and cancellation of official bilateral debt; and more generous ODA for countries committed to poverty reduction. Address the special needs of landlocked developing countries and small island developing States (through the Programme of Action for the Sustainable Development of Small Island Developing States and the outcome of the twenty-second special session of the General Assembly). Deal comprehensively with the debt problems of developing countries through national and international measures in order to make debt sustainable in the long term. In cooperation with pharmaceutical companies, provide access to affordable essential drugs in developing countries. In cooperation with the private sector, make available the benefits of new technologies, especially information and communications. 


\section{Appendix I. Armenia: Debt Sustainability Analysis}

This appendix, jointly drafted by the Fund and Bank staffs, presents joint IMF-World Bank debt sustainability analysis (DSA) for Armenia using the Debt Sustainability Framework for Low-Income Countries (LIC). ${ }^{1}$ Based on the external LIC DSA, Armenia is assessed to be at a low risk of debt distress, with all debt indicators below the relevant country-specific thresholds, including when subject to stress tests. ${ }^{2}$ The public sector DSA suggests that Armenia's overall public sector debt is sustainable, although the debt-to-GDP ratio increased from 16 percent in 2008 to 41 percent in 2009, and is projected to reach about 50 percent in 2012 before gradually decreasing. Following a significant increase in debt, a fiscal consolidation - recently agreed in the context of the EFF/ECF arrangement, as well as the series of World Bank Development Policy Operations - is key to preserve debt and fiscal sustainability over the long term. Implementation of structural reforms would also be critical to boost competitiveness and exports as well as to sustain growth.

\section{Background}

Armenia's total public and private external debt increased during 2005-08, from $\$ 1.45$ billion to $\$ 2.25$ billion. At end-2005, public and publicly-guaranteed (PPG) debt was $\$ 1.1$ billion; this increased to $\$ 1.6$ billion by end 2008 (Table 1). From the equivalent of $\$ 100$ million in 2005 , domestic public debt increased to $\$ 300$ million in 2008 . The share of short-term domestic public debt increased from 13 percent of total public domestic debt in 2005 to 33 percent in 2008, before contracting during the crisis and stabilizing at about 30 percent in 2010 . The text table shows that during 2005-10, on average, 85 percent of Armenia's PPG resulted from government borrowing with the remaining 15 percent coming from central bank borrowing. The latter has been mainly related to the IMF and the German KfW, with the IBRD also contributing a small share during the crisis.

\footnotetext{
${ }^{1}$ The debt data underlying this analysis were provided by the Armenian authorities and donor partners. Data beyond end-2009 are staffs' and authorities' projections.

${ }^{2}$ Armenia is classified as a strong performer based on its three-year average score of 4.32 on the World Bank's Country Policy and Institutional Assessment (CPIA), which measures the country's strength of its policies and institutions. For a strong performer (defined as those with three-year average CPIA ratings above 3.75), the indicative thresholds (for a country where remittance inflows are high) for external debt sustainability are PV of debt-to-GDP + remittances ratio of 45 percent, PV of debt-to-exports + remittances ratio of 180 percent, PV of debt-to-revenue ratio of 300 percent, debt service-to-exports + remittances ratio of 22.5 percent, and debt service-to-revenue ratio of 35 percent.
} 


\begin{tabular}{|c|c|c|c|c|c|c|}
\hline \multicolumn{7}{|c|}{ Armenia: Composition of Armenia PPG in Recent Years, 2005-10 } \\
\hline & 2005 & 2006 & 2007 & 2008 & 2009 & 2010 \\
\hline STOCK OUTSTANDING (mIn USD) & 1,099 & 1,206 & 1,449 & 1,577 & 2,967 & 3,336 \\
\hline \multicolumn{7}{|c|}{ (In percent of total debt stock outstanding) } \\
\hline Government External Borrowings & 0.82 & 0.85 & 0.87 & 0.89 & 0.83 & 0.83 \\
\hline Multilateral & 0.73 & 0.75 & 0.72 & 0.70 & 0.53 & 0.54 \\
\hline IBRD & 0.01 & 0.01 & 0.00 & 0.00 & 0.01 & 0.02 \\
\hline IDA & 0.68 & 0.70 & 0.67 & 0.64 & 0.39 & 0.36 \\
\hline ADB & 0.00 & 0.00 & 0.00 & 0.01 & 0.04 & 0.05 \\
\hline IMF & 0.00 & 0.00 & 0.00 & 0.00 & 0.05 & 0.08 \\
\hline Other multilaterals & 0.00 & 0.00 & 0.00 & 0.00 & 0.00 & 0.00 \\
\hline Bilateral & 0.09 & 0.09 & 0.15 & 0.19 & 0.30 & 0.29 \\
\hline Russia & 0.00 & 0.00 & 0.00 & 0.00 & 0.17 & 0.15 \\
\hline Germany-KfW & 0.05 & 0.05 & 0.05 & 0.05 & 0.03 & 0.03 \\
\hline USA & 0.04 & 0.03 & 0.03 & 0.02 & 0.01 & 0.01 \\
\hline JICA & 0.00 & 0.00 & 0.06 & 0.11 & 0.09 & 0.10 \\
\hline Central Bank External Borrowings & 0.18 & 0.15 & 0.13 & 0.11 & 0.17 & 0.17 \\
\hline Multilateral & 0.16 & 0.14 & 0.11 & 0.09 & 0.15 & 0.16 \\
\hline IMF & 0.16 & 0.14 & 0.11 & 0.09 & 0.14 & 0.15 \\
\hline IBRD & 0.00 & 0.00 & 0.00 & 0.00 & 0.01 & 0.01 \\
\hline Bilateral (Germany-KfW) & 0.02 & 0.02 & 0.02 & 0.03 & 0.02 & 0.01 \\
\hline Public External Guarantees (EBRD) & 0.00 & 0.00 & 0.00 & 0.00 & 0.00 & 0.00 \\
\hline
\end{tabular}

Source: Armenian authorities.

The large fiscal deficit in 2009-8 percent of GDP—contributed to an increase in the stock of public external borrowing to nearly $\$ 3$ billion. This, combined with a sharp drop of output, left the ratio of total public debt to GDP at 41 percent, up from 16 percent in 2008. The public debt ratio is projected to increase further to 44 percent in 2010. Before the crisis, IDA credits were providing the bulk of government borrowing. During the crisis, the IMF and the ADB scaled up their government financing to 9 percent of the 2009 total debt stock, and 13 percent of the 2010 total debt stock. IBRD contribution also increased, reaching 2 percent of 2010 debt stock. Among bilateral lenders, Russia and Japan also scaled up their financing during the crisis, increasing the share of the bilateral PPG to 30 percent of total PPG debt stock, from 19 percent in 2008. 


\section{Box I.1. Armenia: Key Macroeconomic Assumptions for Baseline Scenario (2010-30)}

Real GDP growth is projected to be 4 percent for 2010 and 4.6 percent for 2011 , after which it will gradually return to its medium-term potential growth rate of about 4 percent per year during 2012-15 (below the 10-year historical average of 8.7 percent), and will remain at that level thereafter. Near-term growth will be supported by the robust activity in industry and services, facilitated by a renewal of private transfers and FDI inflows.

For 2010, inflation is expected to be around 7 percent by year-end, due largely to sharp price increases for food products. Inflation is expected to revert to the central bank's target range of $4 \pm 1 \frac{1}{2}$ percent during the second half of 2011 . Consistent with the target range, the average inflation rate over the long term is assumed to be around 4 percent.

The overall fiscal deficit is projected to decrease from about 8 percent of GDP in 2009 to $2 \frac{1}{2}$ percent by 2013 , in line with understandings under the EFF/ECF-supported program. Over the long term, the deficit is projected to remain between 1-2 percent of GDP.

Net domestic financing (NDF) is expected to remain at around $2 \frac{1}{2}$ percent of GDP by 2013 , due to the expected increase in amortization payments starting in 2013 and the expected drop in external flows. The financing mix will continue to rely more on domestic resources throughout the medium term, and NDF will rise to about 3 percent of GDP in 2015, declining thereafter to an average of about 2 percent of GDP in 2016-30, in line with assumed fiscal adjustment.

The current account deficit is projected to narrow from 16 percent of GDP in 2009 to 14.6 percent of GDP in 2010. It will gradually narrow further to about 8 percent in 2015 as exports and remittances pick up in line with the global recovery. Exports are projected to grow robustly over the medium term, as new investments become operational.

As Armenia continues to grow at about 4 percent over the long term, remittances (the growth of which moves in line with developments in Russia, the largest source) will average around 10 percent of GDP and finance a significant share of the trade deficit.

FDI is expected to average about 9 percent of GDP over the medium term. In the near term, FDI is expected to be concentrated in the communications, electricity, gas, and water sectors. Over the longer term, improvements in the business climate should yield a more diversified FDI structure. Long-run net FDI is projected to average around 6 percent of GDP. 


\section{External DSA}

\section{Baseline Scenario}

The baseline scenario shows a sustainable position (Tables I.1 and I.2). The net present value of external debt would gradually increase over the projection period from 34 percent of GDP in 2009 to about 42 percent of GDP in 2012, reflecting an increase in external borrowing, before reversing and declining to 37.5 percent of GDP in 2015. The stock of external debt is projected to drop further, reaching around 17 percent of GDP by 2030. The net present value of external debt to exports plus remittances is projected at 117 percent in 2010, before dropping steadily to reach 30 percent by 2030 (Table I.4). The external debt service to revenue ratio is projected to increase from 6 percent in 2010 to 18 percent in 2013, and then decrease to reach 4 percent in 2030. Under the baseline scenario, all the debt burden and liquidity indicators remain below their indicative thresholds throughout the projection period.

PPG external debt is likely to remain manageable, despite the slight deterioration of the debt indicators relative to the last DSA. The net present value of external PPG debt is expected to rise from 29 percent of GDP in 2009 to 34 percent of GDP in 2011, reflecting a gradual increase in the share of the fiscal deficit financed by external sources in the medium term, before reverting to about 13 percent in 2030. The net present value of public external debt in percent of exports should fall from 183 percent in 2009 to 40 percent in 2030.

\section{Alternative Scenarios and Stress Tests}

The standard menu of alternative scenarios and bound tests indicates that Armenia's external debt outlook would be most adversely affected by depreciation and nondebt flow shocks (Table I.3, Figure I.1). Under the extreme adverse scenario of a 30-percent depreciation of the dram in 2011, the stress tests indicate that the PV of external debt to GDP plus remittances ratio would increase from 28 percent in 2010 to 43 percent in 2012 and then decrease to 16 percent in 2030. The second most adverse scenario of a negative FDI shock would push the PV of external debt to GDP plus remittances ratio to 42 percent in 2012 and back to 12 percent by 2030 . Under the most extreme scenario of 30 percent depreciation, the

debt service to revenue ratio would increase from 6 percent in 2010 to 27 percent in 2013 and come down to 6 percent by 2030 .

Under all these standard alternative scenarios and bound tests, the external debt ratios remain below the relevant thresholds, except when remittances are not included as a source of debt repayment capacity. However, under some scenarios (export shock, FDI shock, or a combination of real GDP shock and deflation in the United States), the debt-to-export ratio crosses the 200 percent threshold over the medium term, underlining the external risks related to the heavy borrowing by the government during the recent crisis. However, given the relative size of remittances coming from migrants and the diaspora, Armenia is assessed to be at low risk of external debt distress. 


\section{Public Sector DSA}

\section{Baseline Scenario}

The baseline scenario shows a sustainable position (Tables I.1 and I.2). The net present value of public sector debt would gradually increase over the projection period from about 33 percent of GDP in 2009 to about 40 percent of GDP in 2012, reflecting the increase in external borrowing. The stock of debt is then projected to gradually drop, reaching around 19 percent of GDP by 2030. The net present value of debt-to-revenue ratio would increase from 13 percent in 2008 to 188 percent in 2011, before dropping again to about 72 percent by 2030. Taking into consideration the legal requirement in Armenia to keep public debt lower than 50 percent of the previous year's GDP, the indicators for public debt would remain at comfortable levels throughout the projection period, and below their indicative thresholds.

\section{Alternative Scenarios and Stress Tests}

The standard menu of alternative scenarios and bound tests indicates that Armenia's public debt outlook would be most adversely affected by a lasting shock to economic growth (Table I.4, Figure I.2). Under the extreme adverse growth scenario, stress tests indicate that public debt ratios would follow a persistent upward trend through the projection horizon. The second most extreme adverse scenario is a 30 percent depreciation in 2011 , which would push the net present value of debt to 55 percent in 2011 before slowly bringing it back to 31 percent by 2030. These two adverse scenarios also worsen Armenia's liquidity situation, putting the debt service to revenue ratio at 26 percent and 33 percent respectively in 2013 from 11 percent in 2010. This result reinforces the importance of maintaining prudent financial policies and preserving macroeconomic stability in order to safeguard the debt outlook. The implicit contingent liabilities of large infrastructure projects that are being considered by the government should also be carefully analyzed.

\section{Debt Distress Classification and Conclusions}

In the staffs' view, Armenia should be considered at low level of debt distress, based on external debt burden indicators. The public sector DSA suggests that Armenia's overall public sector debt dynamics are sustainable in light of the current size of the domestic debt stock.

The rapid accumulation of public debt since the onset of the global crisis calls for pursuing fiscal consolidation over the medium term. Public external debt was only about 14 percent of GDP at end-2008, but reached 36 percent of GDP at end-2009, and is expected to peak at more than 44 percent of GDP in 2012, before gradually declining over the medium term. The projected debt-to-GDP levels do not appear excessive, and standard stress tests show that Armenia's public external debt remains sustainable. However, rollover risk is high, mainly due to the short maturity of Fund financing with repurchase obligations during 2012-14. 
Armenia's debt dynamics are in line with the assessment made at the time of the request for the EFF/ECF arrangement. Under the baseline scenario, the debt burden will temporarily increase in the medium term as a result of significant financing contracted by the government to counteract the effects of the economic crisis. While debt levels are manageable under a strong policy scenario, the outlook is subject to risks, particularly if remittances become volatile over the medium term. The standard bound tests show that under certain scenarios, the debt-to-GDP ratio and the debt-to-exports ratio could go beyond the threshold over the medium term, before receding gradually. The effect of a permanently lower GDP growth shock is particularly pronounced. 
Table I.1. Armenia: External Debt Sustainability Framework, Baseline Scenario, 2007-30 1/

(In percent of GDP, unless otherwise indicated)

\begin{tabular}{|c|c|c|c|c|c|c|c|c|c|c|c|c|c|c|c|}
\hline & \multicolumn{3}{|c|}{ Actual } & \multirow{2}{*}{\multicolumn{2}{|c|}{$\begin{array}{l}\text { Historical } 0 \text { Standard } \\
\text { Average } 0 \text { Deviation }\end{array}$}} & \multicolumn{6}{|c|}{ Projections } & \multirow[b]{2}{*}{$\begin{array}{l}2010-15 \\
\text { Average }\end{array}$} & \multirow{2}{*}{2020} & \multirow[b]{2}{*}{2030} & \multirow[b]{2}{*}{$\begin{array}{l}2016-3 \mathrm{C} \\
\text { Average }\end{array}$} \\
\hline & 2007 & 2008 & 2009 & & & 2010 & 2011 & 2012 & 2013 & 2014 & 2015 & & & & \\
\hline External debt (nominal) 1/ & 16.0 & 16.7 & 41.5 & & & 45.4 & 50.8 & 51.6 & 50.2 & 47.4 & 46.4 & & 31.4 & 207 & \\
\hline Of which: public and publicly guaranteed (PPG) & 14.0 & 13.6 & 36.1 & & & 39.1 & 43.7 & 44.1 & 42.6 & 40.0 & 39.2 & & 25.2 & 16.2 & \\
\hline Change in external debt & -4.3 & 0.7 & 24.8 & & & 3.8 & 5.4 & 0.8 & -1.4 & -2.8 & -1.1 & & -3.3 & -1.4 & \\
\hline Identified net debt-creating flows & -7.5 & 0.4 & 13.6 & & & 4.4 & 1.5 & 0.5 & -1.0 & -2.5 & -2.4 & & -3.1 & -5.0 & \\
\hline Non-interest current account deficit & 6.1 & 11.5 & 15.3 & 7.0 & 5.1 & 13.5 & 11.3 & 10.4 & 8.9 & 7.2 & 7.0 & & 4.5 & -1.6 & 2.9 \\
\hline Deficit in balance of goods and services & 19.7 & 25.6 & 27.5 & & & 26.9 & 27.2 & 26.7 & 26.0 & 25.0 & 24.5 & & 21.1 & 12.4 & \\
\hline Exports & 19.3 & 15.1 & 15.6 & & & 17.1 & 19.4 & 20.9 & 21.9 & 22.7 & 23.1 & & 25.6 & 32.2 & \\
\hline Imports & 39.0 & 40.7 & 43.1 & & & 44.0 & 46.6 & 47.6 & 47.9 & 47.7 & 47.6 & & 46.7 & 44.5 & \\
\hline Net current transfers (negative $=$ inflow) & -10.3 & -9.8 & -9.5 & -9.4 & 1.2 & -9.6 & -11.5 & -11.6 & -12.1 & -12.5 & -12.3 & & -12.2 & -10.5 & -11.6 \\
\hline Of which: official & -1.0 & -0.6 & -0.9 & & & -0.3 & -1.1 & -0.4 & -0.3 & -0.3 & -0.3 & & -0.8 & -0.4 & \\
\hline Other current account flows (negative = net inflow) & -3.4 & -4.4 & -2.6 & & & -3.8 & -4.3 & -4.8 & -5.1 & -5.3 & -5.1 & & -4.4 & -3.5 & \\
\hline Net FDI (negative = inflow) & $\begin{array}{ll}-0.4 \\
-7.6\end{array}$ & -8.1 & -8.5 & -6.0 & 1.8 & $\begin{array}{l}-0.6 \\
-8.6\end{array}$ & -9.0 & $\begin{array}{l}-4.0 \\
-9.1\end{array}$ & $\begin{array}{l}-3.1 \\
-9.1\end{array}$ & -9.0 & $\begin{array}{l}-3.1 \\
-8.8\end{array}$ & & $\begin{array}{l}-4.4 \\
-7.1\end{array}$ & $\begin{array}{l}-3.0 \\
-3.2\end{array}$ & -6.3 \\
\hline Endogenous debt dynamics $2 /$ & -5.9 & -3.1 & 6.8 & & & -0.5 & -0.8 & -0.8 & -0.8 & -0.7 & -0.6 & & -0.4 & -0.3 & \\
\hline Contribution from nominal interest rate & 0.3 & 0.3 & 0.7 & & & 1.2 & 1.3 & 1.3 & 1.3 & 1.2 & 1.1 & & 0.9 & 0.6 & \\
\hline Contribution from real GDP growth & -1.9 & -0.9 & 3.2 & & & -1.6 & -2.1 & -2.1 & -2.1 & -1.9 & -1.8 & & -1.3 & -0.8 & \\
\hline Contribution from price and exchange rate changes & -4.3 & -2.5 & 2.9 & & & & & & & & & & & & \\
\hline Residual (3-4) 3/ & 3.2 & 0.3 & 11.2 & & & -0.6 & 3.9 & 0.4 & -0.5 & -0.3 & 1.4 & & -0.3 & 3.7 & \\
\hline Of which: exceptional financing & 0.0 & 0.0 & 0.0 & & & 0.0 & 0.0 & 0.0 & 0.0 & 0.0 & 0.0 & & 0.0 & 0.0 & \\
\hline PV of external debt $4 /$ & $\ldots$ & $\ldots$ & 34.1 & & & 37.1 & 41.3 & 41.7 & 40.2 & 38.0 & 37.5 & & 26.2 & 17.4 & \\
\hline In percent of exports & $\ldots$ & $\ldots$ & 217.8 & & & 216.5 & 212.7 & 199.2 & 183.9 & 167.5 & 162.0 & & 102.1 & 54.0 & \\
\hline PV of PPG external debt & $\cdots$ & & 28.7 & & & 30.8 & 34.2 & 34.2 & 32.7 & 30.6 & 30.3 & & 20.0 & 12.8 & \\
\hline In percent of exports & $\ldots$ & $\ldots$ & 183.3 & & & 180.0 & 176.1 & 163.3 & 149.3 & 134.9 & 130.9 & & 78.0 & 39.9 & \\
\hline In percent of government revenues & & & 140.2 & & & 150.1 & 162.8 & 159.8 & 149.6 & 139.1 & 136.1 & & 83.0 & 48.6 & \\
\hline Debt service-to-exports ratio (in percent) & 7.4 & 7.8 & 11.3 & & & 13.4 & 13.5 & 24.5 & 26.7 & 19.7 & 15.4 & & 15.9 & 7.9 & \\
\hline PPG debt service-to-exports ratio (in percent) & 2.9 & 3.1 & 5.4 & & & 7.0 & 6.5 & 13.3 & 18.4 & 10.4 & 6.0 & & 8.3 & 3.5 & \\
\hline PPG debt service-to-revenue ratio (in percent) & 2.9 & 2.3 & 4.1 & & & 5.8 & 6.0 & 130 & 18.4 & 10.7 & 6.3 & & 8.9 & 4.3 & \\
\hline Total gross financing need (in billions of U.S. dollars) & 0.0 & 0.5 & 0.7 & & & 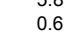 & 0.4 & 0.6 & 0.5 & 0.3 & 0.2 & & $\begin{array}{l}0.9 \\
0.2\end{array}$ & $\begin{array}{c}-0.6 \\
-0.6\end{array}$ & \\
\hline Non-interest current account deficit that stabilizes debt ratio & 10.4 & 10.8 & -9.5 & & & 9.6 & 5.9 & 9.5 & 10.3 & 9.9 & 8.1 & & 7.8 & -0.2 & \\
\hline \multicolumn{16}{|l|}{ Key macroeconomic assumptions } \\
\hline Real GDP growth (in percent) & 13.7 & 6.9 & -14.2 & 8.7 & 8.6 & 4.0 & 4.6 & 4.3 & 4.2 & 4.0 & 4.0 & 4.2 & 4.0 & 4.0 & 4.0 \\
\hline GDP deflator in US dollar terms (change in percent) & 26.8 & 18.4 & -14.7 & 8.3 & 12.9 & -0.6 & -4.2 & -0.4 & 1.3 & 2.1 & 2.1 & 0.1 & 2.1 & 2.1 & 2.1 \\
\hline Effective interest rate (percent) $5 /$ & 2.2 & 2.2 & 2.9 & 2.7 & 0.4 & 2.9 & 2.8 & 2.7 & 2.6 & 2.6 & 2.6 & 2.7 & 2.7 & 2.8 & 2.7 \\
\hline Growth of exports of $G \& S$ (U.S. dollar terms, in percent) & 17.6 & -1.1 & -24.0 & 15.3 & 18.9 & 13.3 & 13.7 & 12.0 & 10.2 & 10.3 & 8.3 & 11.3 & 8.5 & 8.8 & 8.6 \\
\hline Growth of imports of G\&S (U.S. dollar terms, in percent) & 41.5 & 32.3 & -22.4 & 16.5 & 19.8 & 5.6 & 6.2 & 6.2 & 6.2 & 5.7 & 6.2 & 6.0 & 5.8 & 4.8 & 5.8 \\
\hline Grant element of new public sector borrowing (in percent) & & & & $\ldots$ & $\ldots$ & 31.1 & 33.1 & 29.2 & 30.5 & 31.9 & 32.2 & 31.3 & 30.6 & 30.6 & 31.2 \\
\hline Government revenues (excluding grants, in percent of GDP) & 19.3 & 20.1 & 20.4 & & & 20.5 & 21.0 & 21.4 & 21.8 & 22.0 & 22.3 & & 24.1 & 26.4 & 24.8 \\
\hline Aid flows (in billions of U.S. dollars) $6 /$ & 1.1 & 1.1 & 1.6 & & & 1.9 & 2.2 & 2.1 & 2.0 & 2.0 & 1.9 & & 1.4 & 0.4 & \\
\hline Of which: grants & 0.1 & 0.0 & 0.1 & & & 0.1 & 0.1 & 0.0 & 0.0 & 0.0 & 0.0 & & 0.0 & 0.0 & \\
\hline Of which: concessional loans & 1.0 & 1.1 & 1.6 & & & 1.8 & 2.1 & 2.1 & 2.0 & 1.9 & 1.9 & & 1.4 & 0.4 & \\
\hline Grant-equivalent financing (in percent of GDP) 7/ & ... & 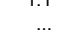 & 1.0 & & & 2.7 & 3.3 & 2.1 & 1.4 & 1.3 & 0.7 & & 0.6 & 0.5 & 0.6 \\
\hline Grant-equivalent financing (in percent of external financing) $7 /$ & $\cdots$ & $\cdots$ & $\cdots$ & & & 45.6 & 50.4 & 34.1 & 37.5 & 39.4 & 47.0 & & 43.8 & 38.3 & 41.5 \\
\hline \multicolumn{16}{|l|}{ Memorandum items: } \\
\hline Nom & 9.2 & 11.7 & 8.5 & & & 8.8 & 8.9 & 9.2 & 9.7 & 10.3 & 11.0 & & 14.8 & 27.1 & \\
\hline Nominal doll & 44.2 & 26.7 & -26.8 & & & 3.4 & 0.3 & 3.8 & 5.6 & 6.2 & 6.2 & 4.3 & 6.2 & 6.2 & 6.2 \\
\hline PV & & & 2.4 & & & 2.6 & 2.9 & 3.1 & 3.1 & 3.2 & 3.2 & & 3.0 & 3.5 & \\
\hline & & & & & & 3.2 & 3.2 & 2.6 & -0.4 & 0.9 & 0.6 & 1.7 & -0.6 & 0.2 & 0.0 \\
\hline & 0.9 & 1.1 & 0.7 & & & 0.8 & 0.9 & 1.0 & 1.1 & 1.3 & 1.3 & & 1.7 & 2.7 & \\
\hline PV of PPG external debt (in percent of GDP + remittances) & $\ldots$ & $\ldots$ & 26.4 & & & 28.2 & 31.0 & 30.8 & 29.2 & 27.3 & 27.0 & & 18.0 & 11.7 & \\
\hline PV of PPG external debt (in percent of exports + remittances) & $\ldots$ & $\ldots$ & 118.3 & & & 116.7 & 114.7 & 106.4 & 97.1 & 87.8 & 86.1 & & 54.1 & 30.4 & \\
\hline Debt service of PPG external debt (in percent of exports + remittances) & $\ldots$ & $\ldots$ & 3.5 & & & 4.5 & 4.2 & 8.6 & 12.0 & 6.8 & 4.0 & & 5.8 & 2.7 & \\
\hline
\end{tabular}

Sources: Amenia a

$1 /$ Includes both public and private sector external debt.

2/Deived as $[r-g-p(1+g) /(1+g+\rho)$.

3/ Includes exceptional financing (i.e., changes in arrears and debt relief); on-lending activities (especially in 2009); changes in gross foreign assets; and valuation adjustments. For

$4 /$ Assumes that PV of private sector debt is equivalent to its face value

$5 /$ Current-year interest payments divided by previous period debt stock.

6/ Defined as grants, concessional loans, and debt relief.

7/ Grant-equivalent financing includes grants provided directly to the government and through new borrowing (difference between the face value and the PV of new debt). 
Table I.2. Armenia: Public Sector Debt Sustainability Framework, Baseline Scenario, 2007-30

(In percent of GDP, unless otherwise indicated)

\begin{tabular}{|c|c|c|c|c|c|c|c|c|c|c|c|c|c|c|c|}
\hline & \multicolumn{3}{|c|}{ Actual } & \multirow[b]{2}{*}{ Average } & \multirow[b]{2}{*}{$\begin{array}{l}\text { Standard } \\
\text { Deviation }\end{array}$} & \multicolumn{5}{|l|}{ Estimate } & \multicolumn{5}{|c|}{ Projections } \\
\hline & 2007 & 2008 & 2009 & & & 2010 & 2011 & 2012 & 2013 & 2014 & 2015 & $\begin{array}{l}2010-15 \\
\text { Average }\end{array}$ & 2020 & 2030 & $\begin{array}{l}2016-30 \\
\text { Average }\end{array}$ \\
\hline $\begin{array}{l}\text { Public sector debt } 1 / \\
\text { Of which: foreign-currency denominated }\end{array}$ & $\begin{array}{l}16.1 \\
14.0\end{array}$ & $\begin{array}{l}16.1 \\
13.6\end{array}$ & $\begin{array}{l}40.6 \\
36.1\end{array}$ & & & $\begin{array}{l}44.0 \\
39.1\end{array}$ & $\begin{array}{l}49.0 \\
43.7\end{array}$ & $\begin{array}{l}49.8 \\
44.1\end{array}$ & $\begin{array}{l}48.7 \\
42.6\end{array}$ & $\begin{array}{l}46.4 \\
40.0\end{array}$ & $\begin{array}{l}45.8 \\
39.2\end{array}$ & & $\begin{array}{l}32.3 \\
25.2\end{array}$ & $\begin{array}{l}22.4 \\
16.2\end{array}$ & \\
\hline Change in public sector debt & -2.6 & 0.0 & 24.5 & & & 3.4 & 5.0 & 0.8 & -1.1 & -2.3 & -0.6 & & -3.1 & -1.4 & \\
\hline Identified debt-creating flows & -3.3 & 0.3 & 13.8 & & & 3.1 & 3.6 & -0.7 & 0.5 & -1.9 & 0.3 & & -1.3 & -0.5 & \\
\hline Primary deficit & 2.0 & 1.5 & 7.2 & 2.2 & 2.3 & 3.6 & 2.6 & 1.6 & 1.1 & 1.2 & 1.0 & 1.8 & 1.0 & 0.7 & 1.1 \\
\hline Revenue and grants & 20.1 & 20.5 & 21.1 & & & 21.8 & 22.7 & 21.8 & 22.2 & 22.4 & 22.6 & & 24.3 & 26.6 & \\
\hline Of which: grants & 0.7 & 0.4 & 0.7 & & & 1.2 & 1.7 & 0.4 & 0.4 & 0.4 & 0.3 & & 0.3 & 0.1 & \\
\hline Primary (noninterest) expenditure & 22.1 & 21.9 & 28.3 & & & 25.4 & 25.3 & 23.4 & 23.3 & 23.6 & 23.6 & & 25.4 & 27.3 & \\
\hline Automatic debt dynamics & -4.9 & -1.5 & 6.7 & & & -0.5 & 1.0 & -2.3 & -0.6 & -3.2 & -0.7 & & -2.3 & -1.2 & \\
\hline Contribution from interest rate/growth differential & -2.5 & -1.3 & 2.8 & & & -1.2 & -1.5 & -1.7 & -1.7 & -1.8 & -1.8 & & -1.5 & -0.8 & \\
\hline Of which: contribution from average real interest rate & -0.2 & -0.2 & 0.1 & & & 0.4 & 0.4 & 0.3 & 0.3 & 0.0 & 0.0 & & -0.2 & 0.1 & \\
\hline Of which: contribution from real GDP growth & -2.3 & -1.0 & 2.7 & & & -1.6 & -1.9 & -2.0 & -2.0 & -1.9 & -1.8 & & -1.4 & -0.9 & \\
\hline Contribution from real exchange rate depreciation & -2.4 & -0.2 & 3.9 & & & 0.7 & 2.6 & -0.6 & 1.1 & -1.3 & 1.1 & & ... & $\ldots$ & \\
\hline Other identified debt-creating flows & -0.5 & 0.3 & 0.0 & & & 0.0 & 0.0 & 0.0 & 0.0 & 0.0 & 0.0 & & 0.0 & 0.0 & \\
\hline Privatization receipts (negative) & -1.4 & -0.9 & 0.0 & & & 0.0 & 0.0 & 0.0 & 0.0 & 0.0 & 0.0 & & 0.0 & 0.0 & \\
\hline Recognition of implicit or contingent liabilities & 0.0 & 0.0 & 0.0 & & & 0.0 & 0.0 & 0.0 & 0.0 & 0.0 & 0.0 & & 0.0 & 0.0 & \\
\hline Debt relief (HIPC and other) & 0.0 & 0.0 & 0.0 & & & 0.0 & 0.0 & 0.0 & 0.0 & 0.0 & 0.0 & & 0.0 & 0.0 & \\
\hline Other (specify, e.g. bank recapitalization) & 0.9 & 1.2 & 0.0 & & & 0.0 & 0.0 & 0.0 & 0.0 & 0.0 & 0.0 & & 0.0 & 0.0 & \\
\hline Residual, including asset changes $2 /$ & 0.8 & -0.3 & 10.7 & & & 0.3 & 1.3 & 1.5 & -1.5 & -0.4 & -0.9 & & -1.8 & -0.9 & \\
\hline \multicolumn{16}{|l|}{ Other Sustainability Indicators } \\
\hline PV of public sector debt & 2.1 & 2.6 & 33.2 & & & 35.7 & 39.5 & 39.9 & 38.7 & 37.0 & 36.9 & & 27.1 & 19.1 & \\
\hline Of which: foreign-currency denominated & 0.0 & 0.0 & 28.7 & & & 30.8 & 34.2 & 34.2 & 32.7 & 30.6 & 30.3 & & 20.0 & 12.8 & \\
\hline Of which: external & & & 28.7 & & & 30.8 & 34.2 & 34.2 & 32.7 & 30.6 & 30.3 & & 20.0 & 12.8 & \\
\hline PV of contingent liabilities (not included in public sector debt) & $\ldots$ & $\ldots$ & $\ldots$ & & & $\ldots$ & & $\ldots$ & $\ldots$ & $\ldots$ & $\ldots$ & & & & \\
\hline Gross financing need $3 /$ & 3.8 & 3.2 & 9.9 & & & 7.1 & 6.4 & 7.1 & 8.0 & 6.4 & 5.2 & & 6.0 & 4.7 & \\
\hline $\mathrm{PV}$ of public sector debt-to-revenue and grants ratio (in percent) & 10.5 & 12.6 & 157.0 & & & 164.1 & 174.1 & 182.7 & 174.4 & 165.3 & 163.2 & & 111.3 & 71.8 & \\
\hline PV of public sector debt-to-revenue ratio (in percent) & 10.9 & 12.9 & 162.2 & & & 173.9 & 188.0 & 186.3 & 177.5 & 168.0 & 165.7 & & 112.5 & 72.2 & \\
\hline Of which: external $4 l$ & & & 140.2 & & & 150.1 & 162.8 & 159.8 & 149.6 & 139.1 & 136.1 & & 83.0 & 48.6 & \\
\hline Debt service-to-revenue and grants ratio (in percent) $5 /$ & 7.5 & 7.4 & 8.2 & & & 10.6 & 10.8 & 18.1 & 23.6 & 15.2 & 10.3 & & 12.0 & 8.0 & \\
\hline Debt service-to-revenue ratio (in percent) $5 /$ & 7.8 & 7.6 & 8.5 & & & 11.2 & 11.7 & 18.5 & 24.1 & 15.4 & 10.5 & & 12.1 & 8.0 & \\
\hline Primary deficit that stabilizes the debt-to-GDP ratio & 4.6 & 1.4 & -17.3 & & & 0.3 & -2.4 & 0.8 & 2.1 & 3.5 & 1.6 & & 4.1 & 2.1 & \\
\hline \multicolumn{16}{|l|}{ Key macroeconomic and fiscal assumptions } \\
\hline Real GDP growth (in percent) & 13.7 & 6.9 & -14.2 & 8.7 & 8.6 & 4.0 & 4.6 & 4.3 & 4.2 & 4.0 & 4.0 & 4.2 & 4.0 & 4.0 & 4.0 \\
\hline Average nominal interest rate on forex debt (in percent) & 0.9 & 0.8 & 1.7 & 1.3 & 0.5 & 2.1 & 1.9 & 1.8 & 1.6 & 1.5 & 1.4 & 1.7 & 1.2 & 1.1 & 1.1 \\
\hline Average real interest rate on domestic debt (in percent) & 6.8 & 4.1 & 8.2 & 15.0 & 19.7 & 4.6 & 9.0 & 7.4 & 7.4 & 3.6 & 3.2 & 5.9 & 0.3 & 4.2 & 1.5 \\
\hline Real exchange rate depreciation (in percent, + indicates depreciation) & -17.1 & -1.8 & 24.7 & -3.5 & 13.7 & 1.9 & & $\ldots$ & $\ldots$ & $\ldots$ & $\ldots$ & $\ldots$ & & $\ldots$ & \\
\hline Inflation rate (GDP deflator, in percent) & 4.2 & 5.9 & 1.3 & 3.5 & 2.2 & 7.5 & 3.9 & 4.3 & 4.3 & 4.0 & 4.0 & 4.7 & 4.0 & 4.0 & 4.0 \\
\hline Growth of real primary spending (deflated by GDP deflator, in percent) & 0.3 & 0.1 & 0.1 & 0.1 & 0.1 & -0.1 & 0.0 & 0.0 & 0.0 & 0.1 & 0.0 & 0.0 & 0.1 & 0.0 & 0.1 \\
\hline Grant element of new external borrowing (in percent) & $\ldots$ & $\ldots$ & $\ldots$ & $\ldots$ & $\ldots$ & 31.1 & 33.1 & 29.2 & 30.5 & 31.9 & 32.2 & 31.3 & 30.6 & 30.6 & $\ldots$ \\
\hline
\end{tabular}

\section{$1 /$ Central government gross debt.}

Sources: Armenian authorities; and Fund staff estimates and projections.

2/ Residuals include changes in gross foreign assets and valuation adjustments. For 2009, the large residual is mainly due to the on-lending activities. For projection also includes contribution from price and exchange rate changes. $3 /$ Gross financing need is defined as the primary deficit plus debt service plus the stock of short-term debt at the end of the last period.

$4 /$ Revenues excluding grants.

5/ Debt service is defined as the sum of interest and amortization of medium and long-term debt. 
Table I.3.Armenia: Sensitivity Analysis for Key Indicators of Public and Publicly Guaranteed External Debt, 2010-2030 (In percent)

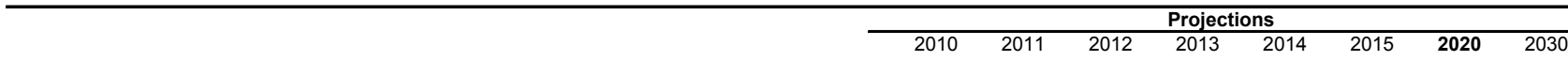

PV of debt-to-GDP+remittances ratio

Baseline

A. Alternative Scenarios

A1. Key variables at their historical averages in 2010-2030 1/

A2. New public sector loans on less favorable terms in 2010-2030 2

\section{B. Bound Tests}

B1. Real GDP growth at historical average minus one standard deviation in 2011-2012

B2. Export value growth at historical average minus one standard deviation in 2011-2012 3/

B3. US dollar GDP deflator at historical average minus one standard deviation in 2011-2012

B4. Net non-debt creating flows at historical average minus one standard deviation in 2011-2012 4/

B5. Combination of B1-B4 using one-half standard deviation shocks

B6. One-time 30 percent nominal depreciation relative to the baseline in 2011 5/

28

28

28

31

31

31

PV of debt-to-exports+remittances ratio
Baseline

\section{A. Alternative Scenarios}

A1. Key variables at their historical averages in 2010-2030 1/ A2. New public sector loans on less favorable terms in 2010-2030 2

\section{B. Bound Tests}

B1. Real GDP growth at historical average minus one standard deviation in 2011-2012 B2. Export value growth at historical average minus one standard deviation in 2011-2012 $3 /$

B3. US dollar GDP deflator at historical average minus one standard deviation in 2011-2012

B4. Net non-debt creating flows at historical average minus one standard deviation in 2011-2012 4/

B5. Combination of B1-B4 using one-half standard deviation shocks

B6. One-time 30 percent nominal depreciation relative to the baseline in 2011 5/

\section{PV of debt-to-revenue ratio}

Baseline

160

A1. Key variables at their historical averages in 2010-2030 1/ A2. New public sector loans on less favorable terms in 2010-2030 2

\section{B. Bound Tests}

B1. Real GDP growth at historical average minus one standard deviation in 2011-2012

B2. Export value growth at historical average minus one standard deviation in 2011-2012 3 /

B3. US dollar GDP deflator at historical average minus one standard deviation in 2011-2012

B4. Net non-debt creating flows at historical average minus one standard deviation in 2011-2012 4/

B5. Combination of B1-B4 using one-half standard deviation shocks

B6. One-time 30 percent nominal depreciation relative to the baseline in $20115 /$ $\begin{array}{llllllll}117 & 115 & 106 & 97 & 88 & 86 & \mathbf{5 4} & 30\end{array}$

$\begin{array}{rrrrrrrr}117 & 97 & 86 & 74 & 70 & 68 & \mathbf{5 6} & 68 \\ 117 & 113 & 112 & 103 & 97 & 94 & \mathbf{6 6} & 45\end{array}$

$\begin{array}{lllrrrrl}117 & 110 & 106 & 95 & 88 & 84 & \mathbf{5 4} & 30 \\ 117 & 130 & 151 & 136 & 127 & 121 & \mathbf{8 2} & 39 \\ 117 & 110 & 106 & 95 & 88 & 84 & \mathbf{5 4} & 30 \\ 117 & 144 & 158 & 128 & 119 & 114 & \mathbf{7 8} & 32 \\ 117 & 145 & 166 & 140 & 131 & 125 & \mathbf{8 6} & 36 \\ 117 & 110 & 106 & 95 & 88 & 84 & \mathbf{5 4} & 30\end{array}$

$\begin{array}{rrrrrrr}131 & 118 & 101 & 95 & 91 & \mathbf{6 8} & 86 \\ 161 & 168 & 158 & 154 & 148 & \mathbf{1 0 1} & 72\end{array}$

$\begin{array}{rrrrrrrr}150 & 163 & 173 & 159 & 152 & 144 & \mathbf{9 0} & 53 \\ 150 & 166 & 187 & 172 & 165 & 157 & \mathbf{1 0 3} & 50 \\ 150 & 157 & 167 & 153 & 146 & 139 & \mathbf{8 7} & 51 \\ 150 & 183 & 212 & 197 & 189 & 180 & \mathbf{1 2 0} & 51 \\ 150 & 169 & 192 & 178 & 171 & 163 & \mathbf{1 0 8} & 47 \\ 150 & 227 & 231 & 212 & 203 & 193 & \mathbf{1 2 1} & 71\end{array}$


Table I.3.Armenia: Sensitivity Analysis for Key Indicators of Public and Publicly Guaranteed External Debt, 2010-2030 (continued)

(In percent)

\section{Debt service-to-exports+remittances ratio}

\section{Baseline}

\section{A. Alternative Scenarios}

A1. Key variables at their historical averages in 2010-2030 1/

A2. New public sector loans on less favorable terms in 2010-2030 2

4

\section{B. Bound Tests}

B1. Real GDP growth at historical average minus one standard deviation in 2011-2012

B2. Export value growth at historical average minus one standard deviation in 2011-2012 3/

B3. US dollar GDP deflator at historical average minus one standard deviation in 2011-2012

B4. Net non-debt creating flows at historical average minus one standard deviation in 2011-2012 4/

B5. Combination of B1-B4 using one-half standard deviation shocks

B6. One-time 30 percent nominal depreciation relative to the baseline in $20115 /$

\section{Debt service-to-revenue ratio}

\section{Baseline}

A. Alternative Scenarios

A1. Key variables at their historical averages in 2010-2030 1/

A2. New public sector loans on less favorable terms in 2010-2030

\section{B. Bound Tests}

B1. Real GDP growth at historical average minus one standard deviation in 2011-2012

B2. Export value growth at historical average minus one standard deviation in 2011-2012 3/

B3. US dollar GDP deflator at historical average minus one standard deviation in 2011-2012

B4. Net non-debt creating flows at historical average minus one standard deviation in 2011-2012 4/

B5. Combination of B1-B4 using one-half standard deviation shocks

B6. One-time 30 percent nominal depreciation relative to the baseline in $20115 /$

Memorandum item

Grant element assumed on residual financing (i.e., financing required above baseline) $6 /$

$\begin{array}{rrrr}5 & 4 & 7 & 9 \\ 5 & 4 & 9 & 12 \\ & & & \\ 5 & 4 & 9 & 12 \\ 5 & 5 & 11 & 15 \\ 5 & 4 & 9 & 12 \\ 5 & 5 & 10 & 12 \\ 5 & 5 & 11 & 14 \\ 5 & 4 & 9 & 12\end{array}$

Sources: Armenian authorities; and staff estimates and projections.

1/ Variables include real GDP growth, growth of GDP deflator (in U.S. dollar terms), non-interest current account in percent of GDP, and non-debt creating flows.

2/ Assumes that the interest rate on new borrowing is by 2 percentage points higher than in the baseline., while grace and maturity periods are the same as in the baseline.

3/ Exports values are assumed to remain permanently at the lower level, but the current account as a share of GDP is assumed to return to its baseline level after the shock (implicit an offsetting adjustment in import levels).

$4 /$ Includes official and private transfers and FDI.

5/ Depreciation is defined as percentage decline in dollar/local currency rate, such that it never exceeds 100 percent.

6/ Applies to all stress scenarios except for A2 (less favorable financing) in which the terms on all new financing are as specified in footnote 2. 
Table I.4. Armenia: Sensitivity Analysis for Key Indicators of Public Debt, 2010-2030

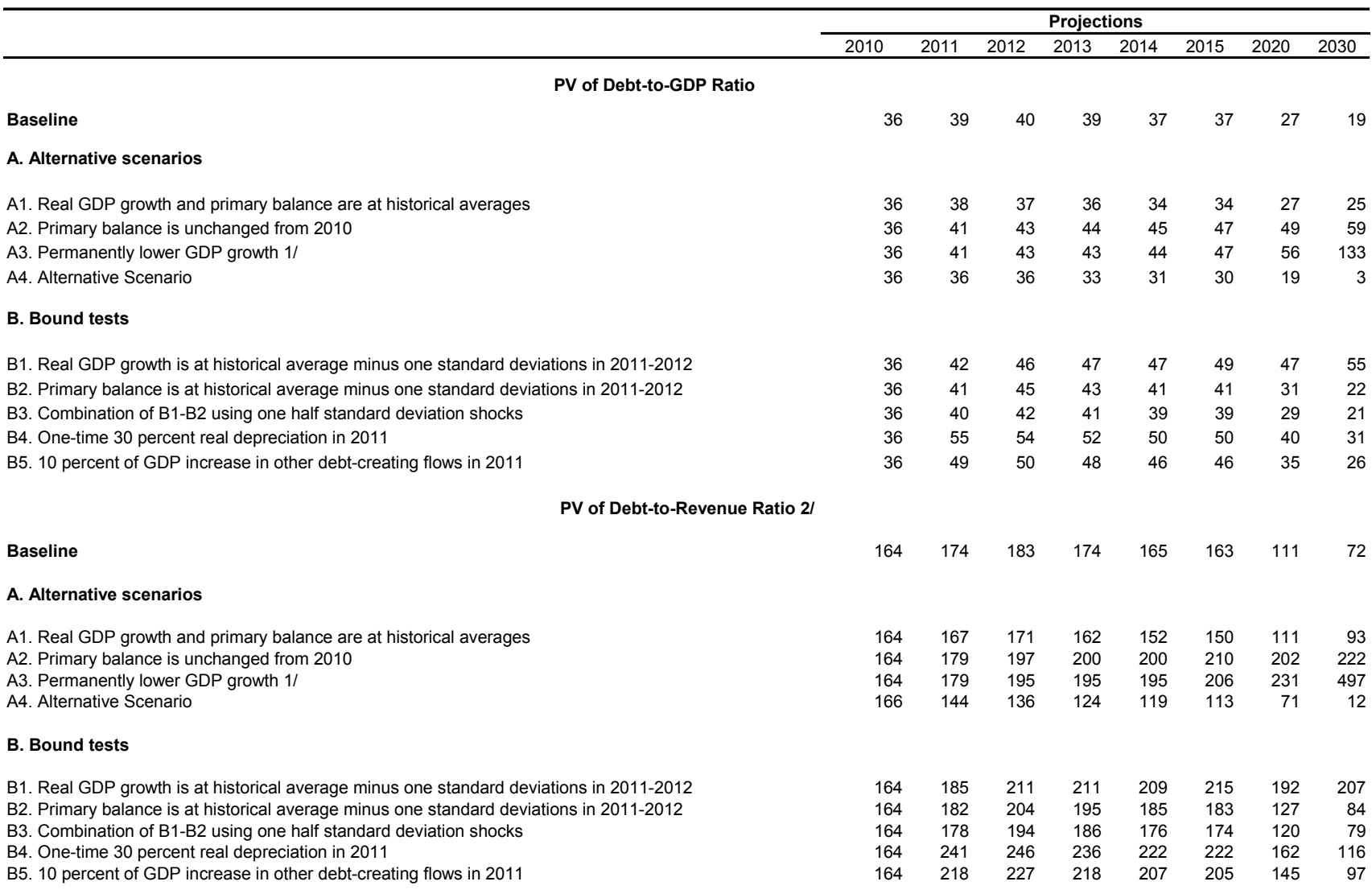

Debt Service-to-Revenue Ratio 2/

Baseline

$\begin{array}{llllllll}11 & 11 & 18 & 24 & 15 & 10 & 12 & 8\end{array}$

A. Alternative scenarios

A1. Real GDP growth and primary balance are at historical averages

A2. Primary balance is unchanged from 2010

A3. Permanently lower GDP growth $1 /$

A4. Alternative Scenario

$\begin{array}{rrrrrrrr}11 & 10 & 17 & 21 & 13 & 9 & 9 & 7 \\ 11 & 11 & 18 & 24 & 16 & 12 & 15 & 17 \\ 11 & 11 & 19 & 25 & 17 & 12 & 18 & 30 \\ 11 & 9 & 14 & 18 & 11 & 7 & 9 & 4\end{array}$

\section{B. Bound tests}

B1. Real GDP growth is at historical average minus one standard deviations in 2011-2012

B2. Primary balance is at historical average minus one standard deviations in 2011-2012

B3. Combination of B1-B2 using one half standard deviation shocks

B4. One-time 30 percent real depreciation in 2011

B5. 10 percent of GDP increase in other debt-creating flows in 2011

Sources: Armenian authorities; and staff estimates and projections.

$1 /$ Assumes that real GDP growth is at baseline minus one standard deviation divided by the square root of the length of the projection period.

2/ Revenues are defined inclusive of grants. 
Figure I.1. Armenia: Indicators of Public and Publicly-Guaranteed External Debt under Alternatives Scenarios, 2010-2030 1/

a. Debt Accumulation
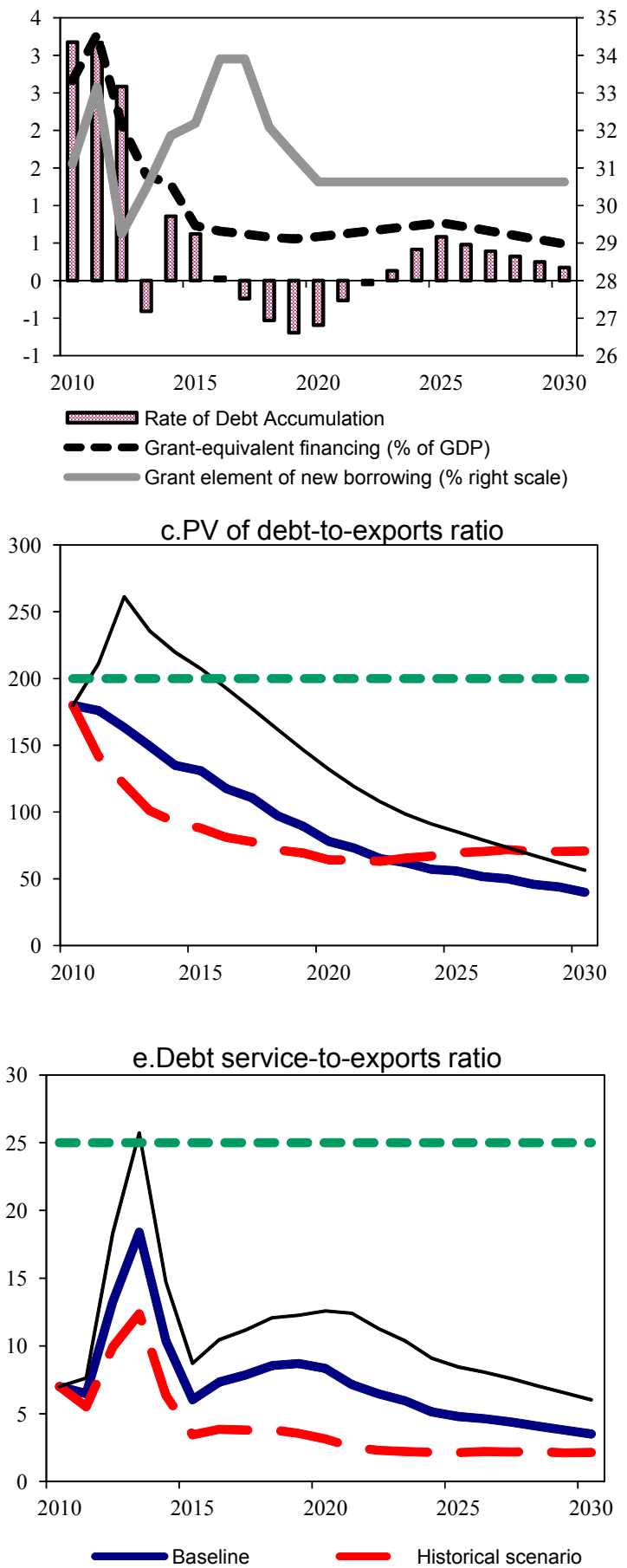
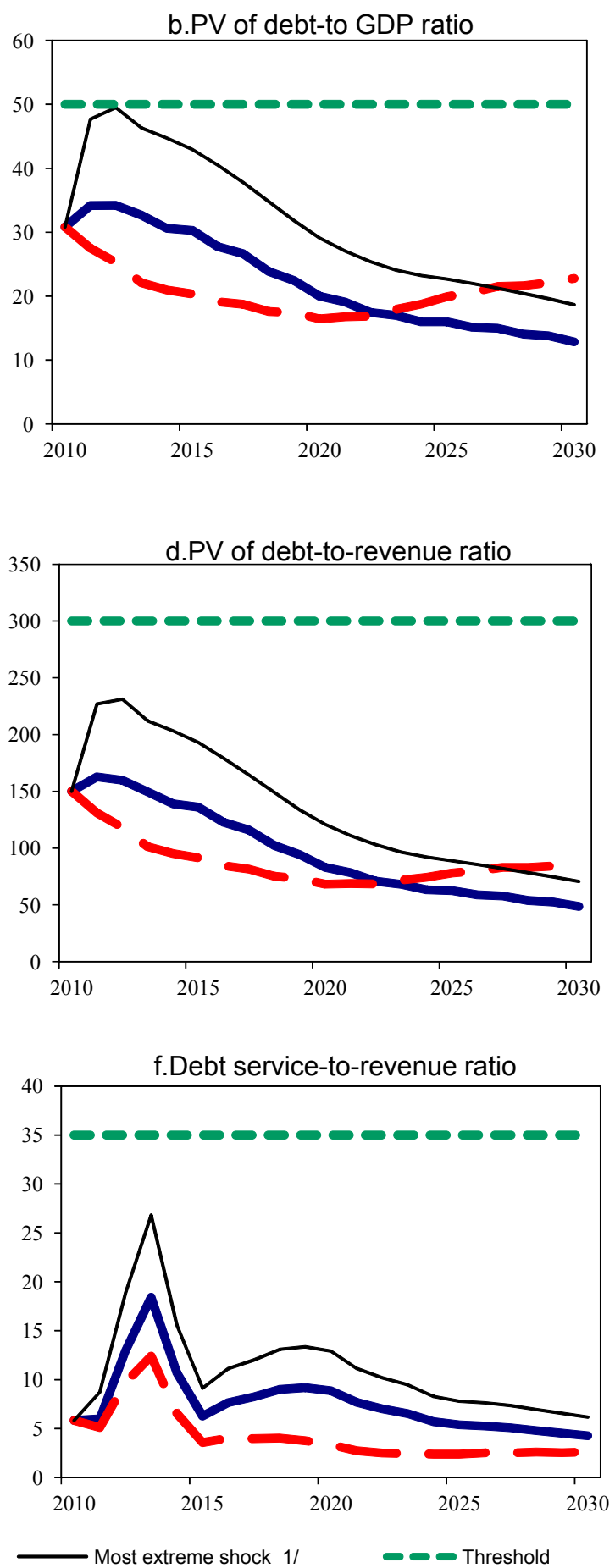

Sources: Armenian authorities; and Fund staff estimates and projections.

1/ The most extreme stress test is the test that yields the highest ratio in 2020. In figure b. it corresponds to a One-time depreciation shock; in c. to a Exports shock; in d. to a One-time depreciation shock; in e. to a Exports shock and in figure f. to a One-time depreciation shock 
Figure I.2. Armenia: Indicators of Public Debt Under Alternative Scenarios, 2010-2020 1/
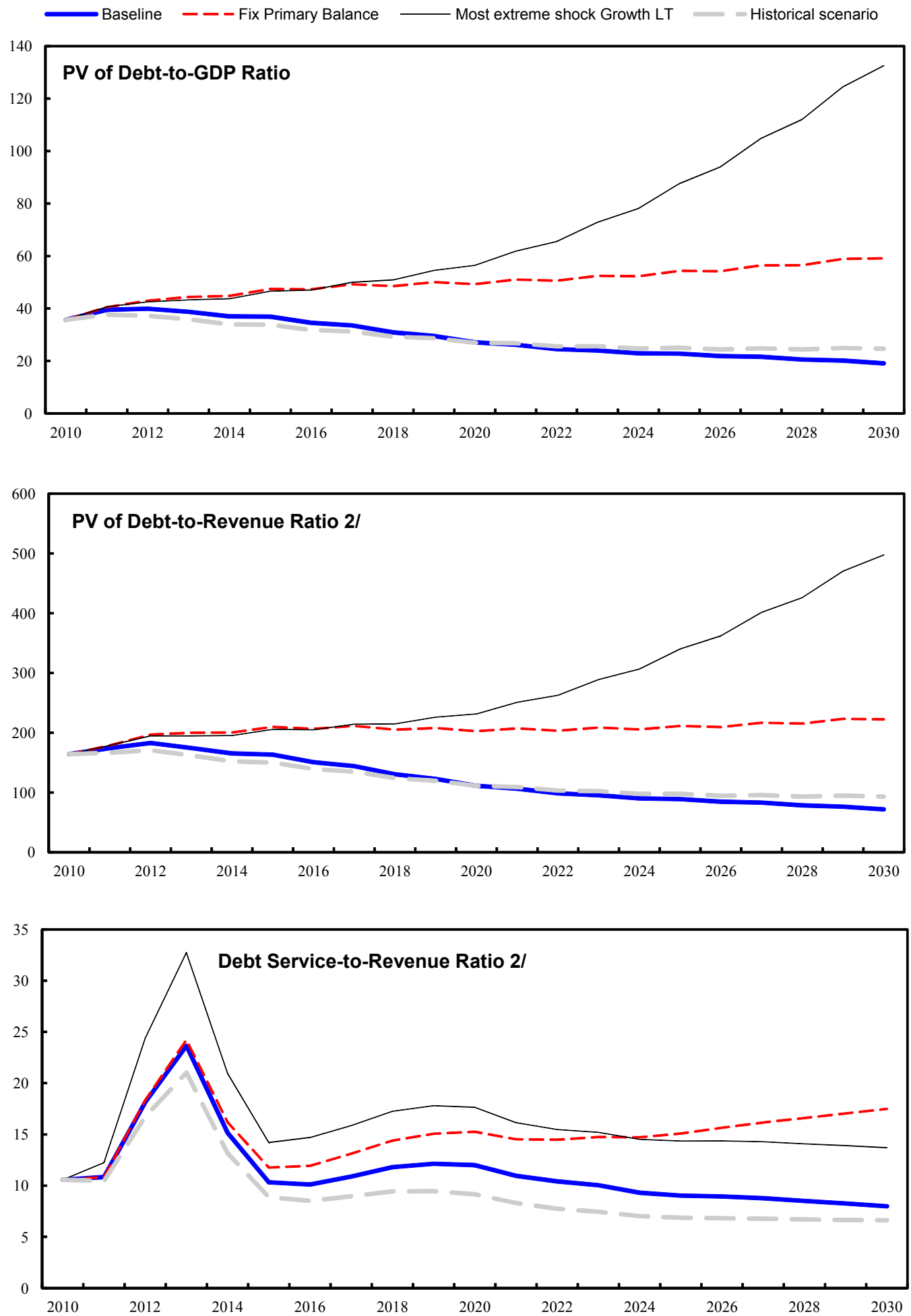

Sources: Armenian authorities; and staff estimates and projections.

$1 /$ The most extreme stress test is the test that yields the highest ratio in 2020.

2/ Revenues are defined inclusive of grants. 


\section{Appendix II. Armenia: Assessment of the Real Exchange Rate and Competitiveness}

Staff's current assessment, based on several methodologies, indicates that Armenia's real exchange rate is overvalued by $10-12$ percent. The nominal effective exchange rate (NEER) and real effective exchange rate (REER) indices show that the dram has recently appreciated, suggesting that the current overvaluation may be higher. Compared to major trading partners and regional competitors, the dram showed the highest real appreciation between December 2009 and July 2010.
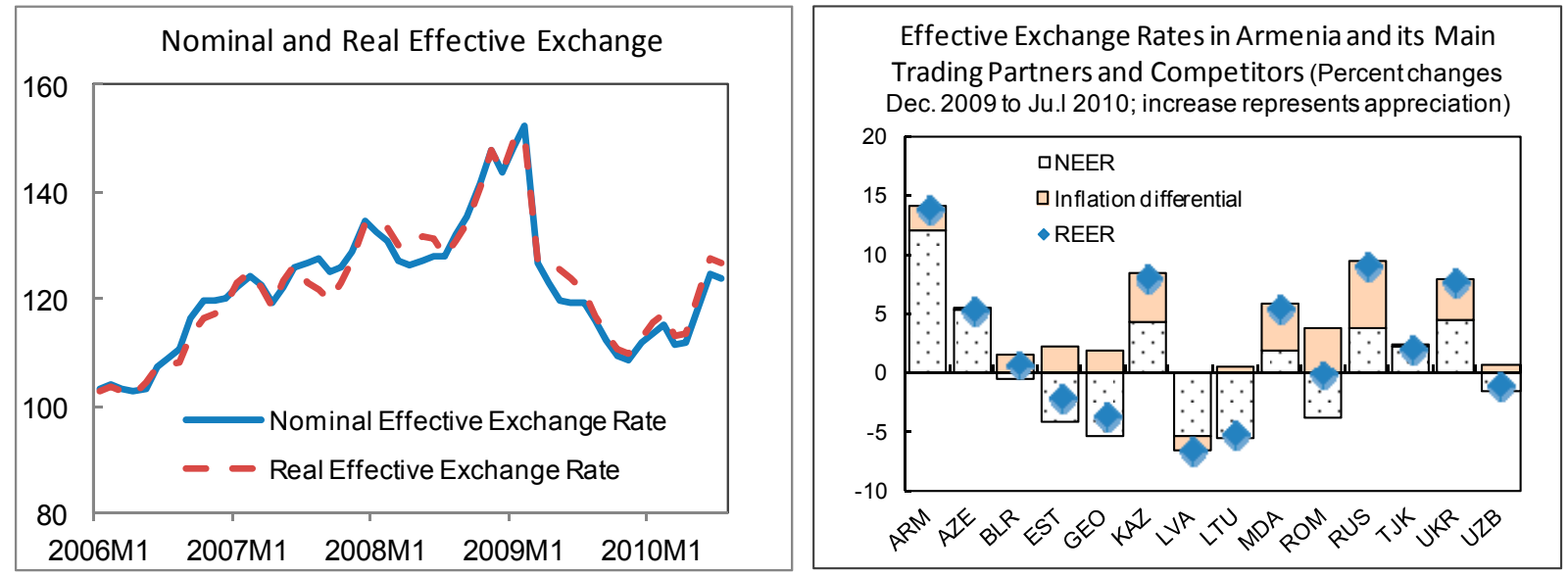

Sources: Armenian authorities; and Fund staff estimates.

Estimates using methodologies of the IMF's Consultative Group on Exchange Rate (CGER) suggest that while the dram is currently overvalued, it will return to its equilibrium value in the medium term (2015), based on policies under the Fundsupported program. The Macroeconomic Balance (MB) Approach, which measures the exchange rate needed to shift the underlying current account to its sustainable level, indicates an overvaluation of about 15 percent in 2009, and based on staff current account projections, predicts an overvaluation of 11 percent in 2010. ${ }^{1}$ The External Sustainability (ES) Approach, which relates the equilibrium exchange rate to the gap between the current account that stabilizes the net external position and the actual current account, suggests an overvaluation of 27.5 and 20.1 percent in 2009 and 2010, respectively, based on current account projections under the program. ${ }^{2}$ Estimates using the Equilibrium Real Exchange Rate (ERER) Approach suggest an overvaluation of 10.9 percent at the end of 2009. ${ }^{3}$ The

\footnotetext{
${ }^{1}$ The MB approach is based on the CGER methodology, but uses only emerging market economies in the crosscountry panel regression. The coefficients obtained from this regression are then applied to data for Armenia.

${ }^{2}$ The net external position is extracted from Lane, P. and Milesi-Ferretti, G. (2007), "The External Wealth of Nations Mark II: Revised and Extended Estimates of Foreign Assets and Liabilities, 1970-2004," Journal of International Economics, Vol. 73(2), pp. 223-250. Data for Armenia have been updated for 2008.

${ }^{3}$ The ERER results presented here are derived from a single, country-specific model developed by Fund staff from the Middle East and Central Asia Department (i.e., they are not drawn from the Fund-wide CGER panel).
} 
ERER approach estimates the long-run statistical relationship between the real exchange rate and its fundamentals, namely, the terms of trade, openness, government consumption and investment, relative real per capita GDP, and the net international investment position. An autoregressive distributed lag (ARDL) model is used to estimate a reduced form relationship between the real effective exchange rate and selected fundamentals.
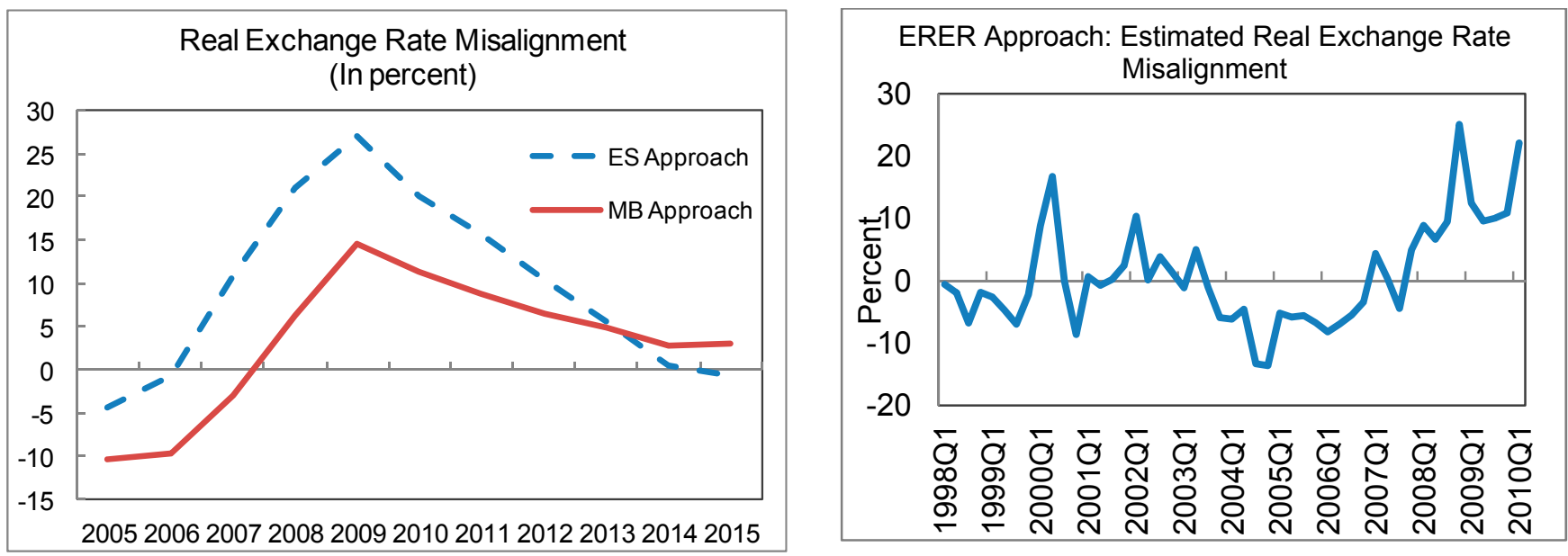

Sources: Armenian authorities; and Fund staff estimates and projections.

\section{Estimates based on a cointegrated panel consisting of transition economies in Europe and Central Asia also point to an overvaluation of around 10 percent. This approach,} which uses the same fundamentals as the ERER approach, allows heterogeneity among countries in the short run while assuming homogeneity only in the long run. ${ }^{4}$ Its advantage lies in its time and cross-section dimensions, which substantially improve inference. The panel is estimated using common correlated effects estimators, which augment explanatory variables by cross-section averages to capture the impact of common factors and thereby yield consistent and asymptotically normal parameter estimates. Both the common correlated effects mean group estimator (CCEMG) and the common correlated effects pooled (CCEP) estimator are computed. They point to an overvaluation of the dram of 9.6 and 12.4 percent at end-2009, respectively.

The CGER ERER panel does not include Armenian data, while productivity data used in the CGER regression are not directly comparable to data available for Armenia, so that coefficients of the CGER ERER could not easily be applied to Armenian data.

${ }^{4}$ Following the methodology of Hashem Pesaran (2006), "Estimation and Inference in Large Heterogeneous Panels with a Multifactor Error Structure," Econometrica, Vol. 74(4), pp. 967-1012. 

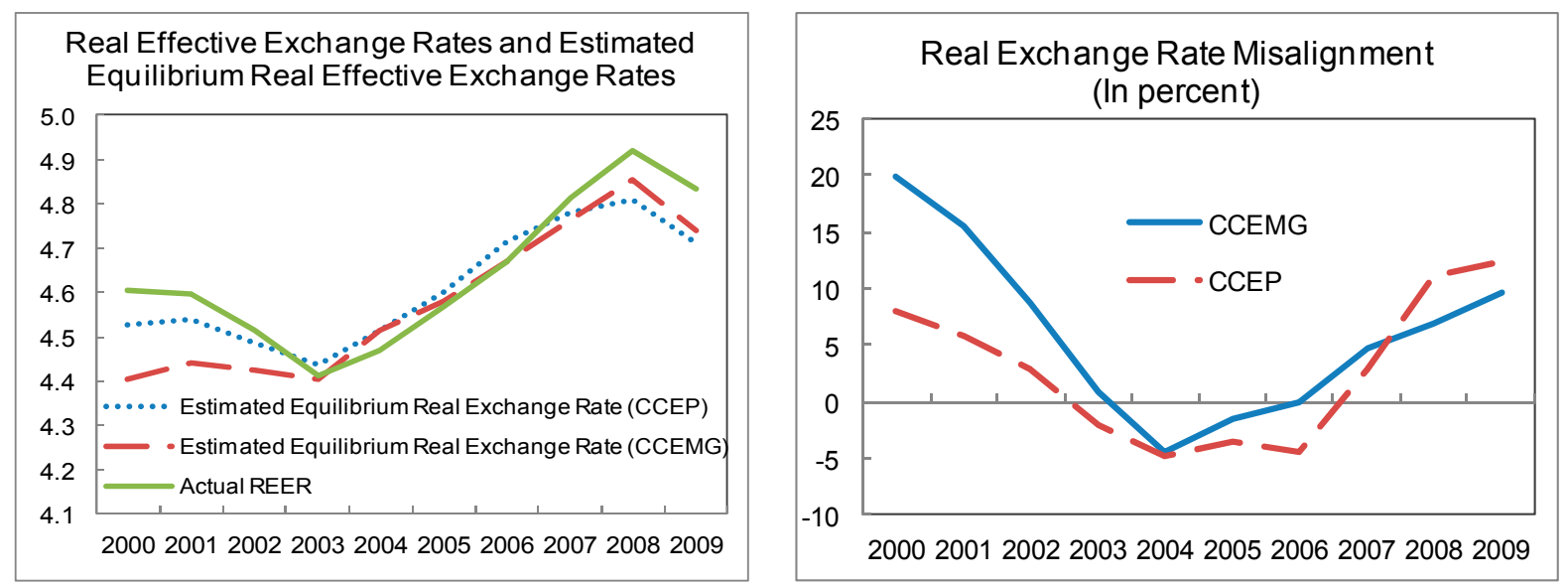

Sources: Armenian authorities; and Fund staff estimates and projections.

Four out of five estimators indicate that the dram is currently overvalued by 10-12 percent; these results are in line with estimates of the Central Bank of Armenia. Only the results from the ES approach point to an overvaluation in the 20 percent range and thus represent an outlier compared to the other four estimators. Real exchange rate estimates of the CBA, which are based on an error correction model with remittances, capital inflows, and government investment as long-run explanatory variables, also point to an exchange rate overvaluation of 9-12 percent in mid-2010. As such and because of data limitations for the ES approach (data on the net external position are not available after 2008), current overvaluation of around 10-12 percent is viewed as the most reliable range. 


\section{Appendix III. Armenia: Tax Performance in Armenia and Its Peers}

Armenia's tax-to-GDP ratio is low relative to comparator countries (text figure). ${ }^{1}$ At about 17 percent in 2008, Armenia's tax-to-GDP ratio is low relative to that of its regional peers, including those with lower per capita income (Georgia, Kyrgyz Republic, Moldova, Mongolia, Ukraine, and Uzbekistan). ${ }^{2}$ This suggests that there is revenue potential that remains to be tapped.

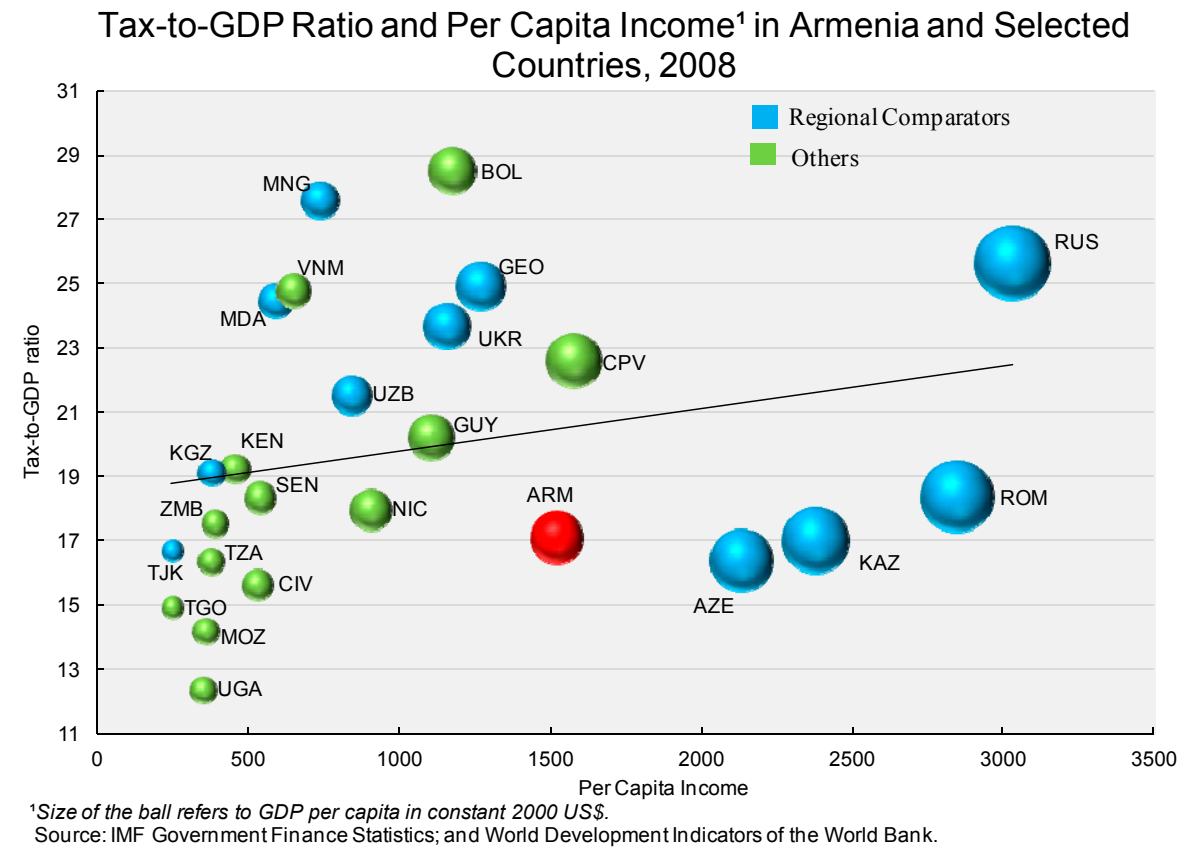

\section{Weaknesses in taxes on income and on goods and services explain most of the gap}

(Table III.1). Taxes on goods and services (goods tax) and taxes on income, profits, and capital gains (income tax) contribute the main share of tax revenue in all countries.

Armenia's performance with respect to both groups of taxes lags behind regional peers, including countries with lower per capita income and noncommodity exporters. Armenia's weaknesses seem to be more prevalent in collecting income taxes (about 3 percentage points of GDP) than taxes on goods and services (about 1 percentage point of GDP). Tax reform should focus on both tax types, with particular emphasis on addressing weaknesses in income tax collection.

\footnotetext{
${ }^{1}$ The broader set of comparators include Azerbaijan, Belarus, Estonia, Georgia, Kazakhstan, Latvia, Lithuania, Moldova, Mongolia, Romania, Russia, Tajikistan, Ukraine, and Uzbekistan. However, lack of data in some cases limits the comparison to a subset of these countries.

${ }^{2}$ In 2008, Georgia collected about 25 percent of GDP in tax revenues, including social contributions-which was unified with the income tax starting 2008.
} 
Table III.1: Tax Revenues in Armenia and Selected Regional Countries (2006-08)

\begin{tabular}{|c|c|c|c|c|c|}
\hline & $\begin{array}{l}\text { Total Tax } \\
\text { Revenue }\end{array}$ & Goods & Income & Trade & Other \\
\hline Armenia & 15.7 & 8.8 & 4.7 & 0.8 & 1.4 \\
\hline Azerbaijan & 16.9 & 5.5 & 9.6 & 1.1 & 0.6 \\
\hline Belarus & 35.6 & 17.4 & 8.0 & 5.8 & 4.5 \\
\hline Estonia & 20.6 & 12.7 & 7.6 & 0.0 & 0.3 \\
\hline Georgia & 21.9 & 13.3 & 7.2 & 0.5 & 0.9 \\
\hline Kazakhstan & 27.0 & 5.2 & 8.5 & 1.6 & 11.7 \\
\hline Kyrgyz & 18.5 & 10.3 & 3.3 & 2.5 & 2.3 \\
\hline Lithuania & 20.8 & 11.4 & 9.4 & 0.0 & 0.1 \\
\hline Moldova & 23.7 & 16.7 & 4.5 & 1.8 & 0.8 \\
\hline Mongolia & 28.0 & 4.1 & 13.2 & 7.0 & 3.7 \\
\hline Russia & 26.5 & 7.0 & 9.6 & 8.0 & 1.9 \\
\hline Tajikistan & 15.7 & 10.8 & 2.4 & 1.8 & 0.7 \\
\hline Ukraine & 23.1 & 11.6 & 9.7 & 1.5 & 0.3 \\
\hline Uzbekistan & 21.1 & 12.5 & 6.5 & 0.9 & 1.3 \\
\hline Regional Comparators 1/ & 22.7 & 10.7 & 7.5 & 2.3 & 2.1 \\
\hline with lower income 2/ & 21.7 & 11.3 & 6.7 & 2.3 & 1.4 \\
\hline with higher income 3/ & 23.7 & 10.1 & 8.4 & 2.4 & 2.8 \\
\hline Non-commodity exporters $4 /$ & 22.4 & 12.1 & 6.7 & 1.5 & 2.2 \\
\hline Armenia 2008 & 16.8 & 9.6 & 4.7 & 1.0 & 1.4 \\
\hline \multicolumn{6}{|c|}{$\begin{array}{l}\begin{array}{c}\text { Difference between Armenia in } 2008 \text { and the average of selected regional } \\
\text { comparators }\end{array}\end{array}$} \\
\hline Regional Comparators 1/ & -6.0 & -1.1 & -2.9 & -1.3 & -0.7 \\
\hline with lower income 2/ & -5.0 & -1.7 & -2.0 & -1.2 & 0.0 \\
\hline with higher income $3 /$ & -7.0 & -0.5 & -3.7 & -1.4 & -1.4 \\
\hline Non-commodity exporter $4 /$ & -5.7 & -2.5 & -2.0 & -0.5 & -0.7 \\
\hline
\end{tabular}

Sources: Armenian authorities; and Fund staff estimates.

1/ Azerbaijan, Belarus, Estonia, Georgia, Kazakhstan, Kyrgyz, Lithuania, Moldova, Mongolia, Tajikistan, Russia, Ukraine, and Uzbekistan.

2/ Georgia, Kyrgyz, Moldova, Mongolia, Tajikistan, Ukraine, and Uzbekistan.

3/ Azerbaijan, Belarus, Estonia, Kazakhstan, Lithuania, and Russia.

4/ Belarus, Estonia, Georgia, Kazakhstan, Kyrgyz, Lithuania, Moldova, Tajikistan, Ukraine, and Uzbekistan. 


\section{An analysis of revenue productivity suggests that VAT productivity in} Armenia, while improved, remains below potential (Table III.2). ${ }^{1} \mathrm{VAT}$ productivity increased from 0.35 percent in 2003 to 0.5 percent in $2008 .^{2}$

However, it remains low compared to most other transition economies, which may reflect policy gaps due to differences in exemptions, as well as compliance gaps owing to differences in administration capacity. Although Armenia's VAT rate is the same as in Belarus, Moldova, and Ukraine, each of these countries has higher collections. If revenue productivity was 0.64 percent, the highest efficiency recorded in the region, Armenia's VAT revenue-to-GDP ratio would have been 10.5 percent, more than 2 percentage points higher.

\section{Despite their increasing share in tax revenues, revenue productivity of income taxes in Armenia remains below that of other transition economies (Table III.3). A comparison of} corporate (CIT) and personal income tax (PIT) productivity across transition economies points to low productivity of income taxes in Armenia. ${ }^{3}$ This reflects policy gaps, such as tax exemptions to the agriculture sector, tax incentives to large companies, and preferential tax regimes for certain sectors (fuel and tobacco). Low productivity of income taxes could also be attributed to compliance gaps, owing to weak tax administration and tax evasion, including under-declaration of wages and employment.

\footnotetext{
${ }^{1}$ Revenue productivity reflects the effectiveness or efficiency of revenue collection given the prevailing standard tax rate. It measures how much revenue relative to the tax base each percentage point of the tax rate provides. It is calculated by taking the actual revenue collected as a percentage of the tax base (=tax yield), divided by the tax rate.

${ }^{2}$ The estimated VAT revenue productivity uses consumption as the tax base. It also includes VAT tax credits, and thus overstates the "actual" productivity. VAT revenue productivity, excluding VAT tax credits in 2008 is 0.33 percent, up from 0.27 in 2006 .

${ }^{3}$ The estimated revenue productivity for income taxes includes profit and income tax credits, and thus overstates the "actual" productivity. CIT revenue productivity, excluding CIT tax credits in 2008 is 0.08 percent, down from 0.10 percent in 2006. PIT revenue productivity, excluding PIT tax credits in 2008 is 0.07 percent, up from 0.06 percent in 2006 .
} 
Table III.3: Income Tax Rates, Revenues, and Productivity in Armenia and Selected Transition Countries, 2008

\begin{tabular}{|c|c|c|c|c|c|c|}
\hline & \multicolumn{3}{|c|}{ Corporate Income Tax } & \multicolumn{3}{|c|}{ Personal Income Tax } \\
\hline & \multicolumn{3}{|c|}{2008} & \multicolumn{3}{|c|}{2008} \\
\hline & Tax Rate 1/ & $\begin{array}{c}\text { Revenue } \\
\text { (Percent of } \\
\text { GDP) }\end{array}$ & $\begin{array}{c}\text { Revenue } \\
\text { Productivity 2/ }\end{array}$ & Tax Rate 1/ & $\begin{array}{c}\text { Revenue } \\
\text { (Percent of } \\
\text { GDP) }\end{array}$ & $\begin{array}{c}\text { Revenue } \\
\text { Productivity 2/ }\end{array}$ \\
\hline Armenia & 20 & 2.4 & 0.12 & $10-20$ & 1.5 & 0.10 \\
\hline Azerbaijan & 22 & 7.1 & 0.32 & $14-35$ & 1.6 & 0.06 \\
\hline Belarus & $\ldots$ & 4.9 & $\ldots$ & $\ldots$ & 3.2 & $\ldots$ \\
\hline Estonia & 19 & 1.7 & 0.09 & 19 & 6.3 & 0.33 \\
\hline Georgia & 20 & 3.1 & 0.16 & 20 & 6.8 & 0.34 \\
\hline Kazakhstan & 30 & 5.7 & 0.19 & $5-20$ & 1.6 & 0.13 \\
\hline Latvia & 15 & 3.1 & 0.21 & $15-25$ & 6.3 & 0.32 \\
\hline Lithuania & 15 & 2.8 & 0.18 & $15-27$ & 6.6 & 0.31 \\
\hline Moldova & $\ldots$ & 1.1 & $\ldots$ & $7-18$ & 2.4 & 0.19 \\
\hline Romania & 16 & 2.7 & 0.17 & 16 & 3.6 & 0.23 \\
\hline Russia & $20-24$ & 5.6 & 0.26 & 13 & 3.9 & 0.30 \\
\hline Ukraine & 25 & 5.1 & 0.20 & 15 & 4.9 & 0.33 \\
\hline
\end{tabular}

Sources: Armenian authorities; and Fund staff estimates.

$1 /$ Corporate and income tax rates based on a summary report 2007/08.

2/ When the corporate (personal) income tax applies at different rates, an average of the lowest and highest marginal corporate (personal) income tax rate is used as an approximation to compute revenue productivity 


\section{Appendix IV. Armenia: Business Climate and Competitiveness}

According to reports of the World Economic Forum, Armenia ranks well below most CIS and Eastern European countries on indicators of competitiveness. Armenia was ranked 98 out of 139 countries in 2010 , outperforming only Tajikistan and the Kyrgyz Republic. Key constraints in doing business in 2010 were the lack of local competition, the extent of market dominance, and the ineffectiveness of anti-monopoly policy. On these indicators, Armenia was ranked at the bottom of the sample $(136,133$, and 138 respectively of 139 countries).

Notably, although GDP grew by about 12 percent per year during 2005-08, Armenia's relative international competitiveness declined.

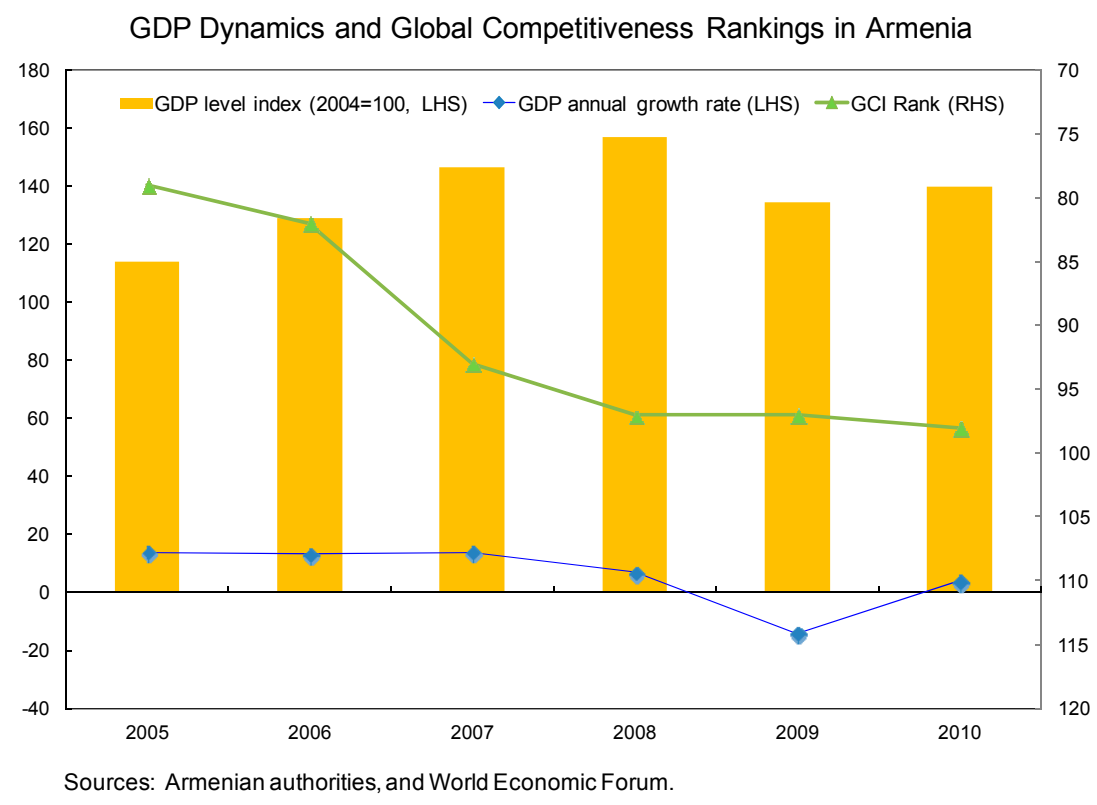

While starting a business in Armenia is relatively easy, conducting business is difficult. Armenia was ranked 21 out of 183 countries in 2010 in the "starting a business" index, but its position in the "protecting investors" and "paying taxes" indices was significantly lower at 
93 and 153, respectively. Furthermore, a majority of businesses indicated that corruption, access to finance, and crime, theft, and disorder have recently emerged as major concerns.
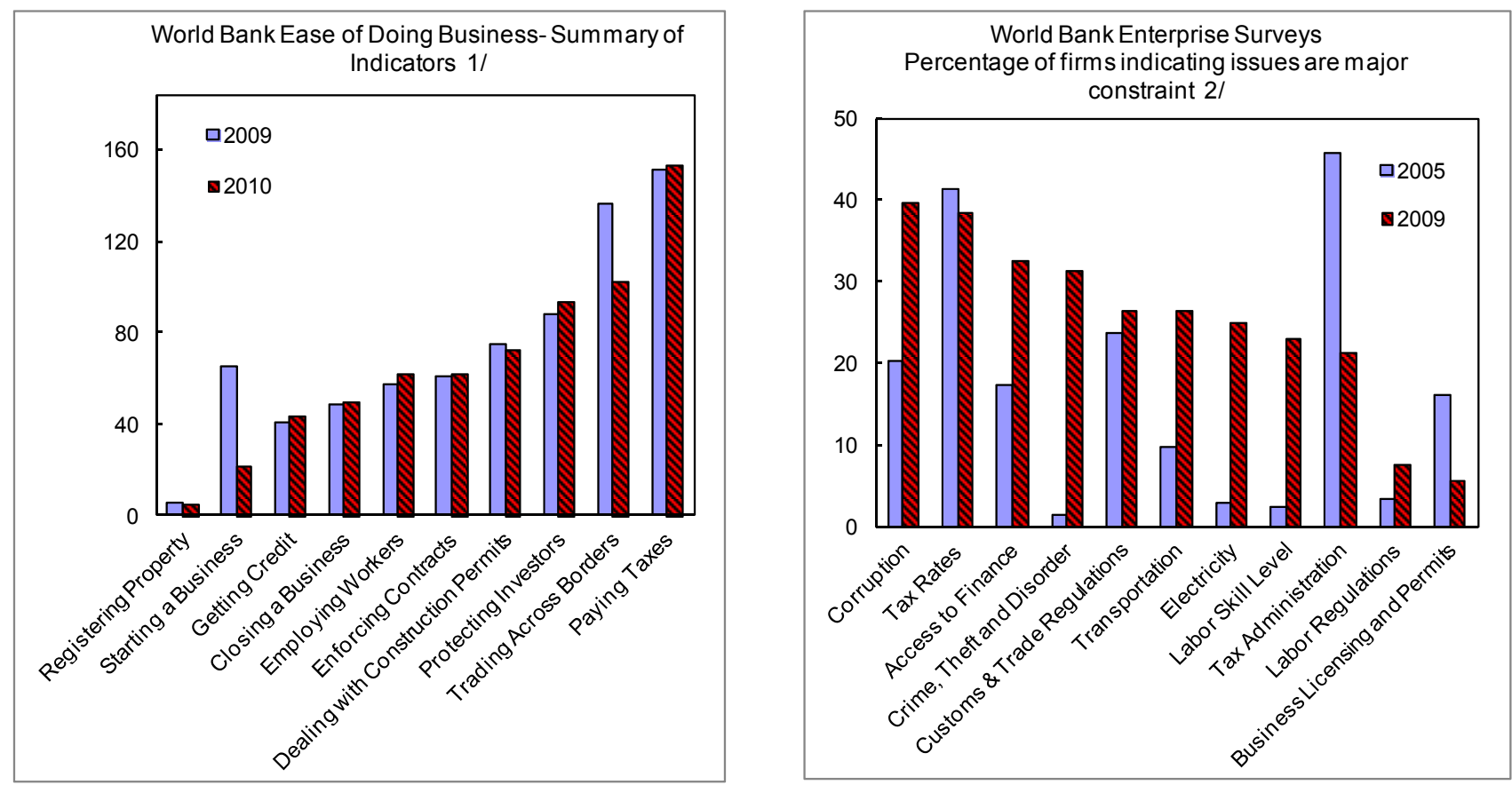

$1 /$ Rankings are out of 183 countries, with a lower score indicating greater ease of doing business.

2/ 302 and 374 enterprises are surveyed in 2005 and 2009 respectively.

The following measures have been taken in 2010 to improve the business environment:

- In order to reduce the cost of business registration, the government approved a decree in September 2010 that creates a one-stop shop for business registration. This decree is expected to become effective in 2011, after parliament approves amendments to the current law.

- The government also approved amendments to different tax laws that simplify the taxpayer reporting system by reducing the frequency of reporting and unifying the deadlines for the filing of different reports. The main goal of these amendments is to strengthen the business environment and improve the "paying taxes" index.

- With the aim to strengthen the autonomy and enforcement capacity of the State Commission for the Protection of Economic Competition (SCPEC), an amendment on competition-related legislation that harmonizes the current legislation with international best practices has been drafted and is under consideration in the government.

- An amendment to the law on inspections, which strengthens the rights of businesses by decreasing the discretionary authority of inspectors, is also under consideration. 
- The Public Service Law, which restricts public officials from engaging in commercial activities, was submitted to parliament in 2009 and passed the first reading in June 2010. 


\section{ATTACHMENT I. ARMENIA: LETTER OF INTENT}

Mr. Dominique Strauss-Kahn

Yerevan, November 11, 2010

Managing Director

International Monetary Fund

Washington, D.C. 20431

Dear Mr. Strauss-Kahn:

1. Armenia has exited the crisis, but the economic recovery is fragile and a swift return to the high pre-crisis economic growth is unlikely. Thus far in 2010, growth has been positive, and public finances have improved considerably. The financial sector continues to be well capitalized and stable. Nonetheless, the economy faces considerable headwinds, including weak external demand, important structural rigidities, and social challenges. Sustained fiscal adjustment is needed, and in the financial sector, continued steps are warranted to deepen intermediation, lower dollarization, and reduce vulnerabilities. At the same time, we will aim at reducing external imbalances and enhancing competitiveness.

\section{Performance under our Extended Fund Facility and Extended Credit Facility} (EFF/ECF) programs has been very strong. The program is on track with all quantitative performance criteria and indicative targets for end-June 2010 as well as all indicative targets for which data are available for end-September 2010 observed. The structural benchmarks were also met, except for the benchmark on the establishment of the tax appeals board which was met with a delay.

3. This Letter of Intent (LOI) builds on the LOI and MEFP agreed between the Armenian authorities and the Fund under the EFF/ECF arrangement approved on June 28, 2010. Our near-term macroeconomic policies will continue to aim at balancing the need to support the ongoing economic recovery and contain eventual macroeconomic pressures. We remain committed to our reform program aimed at economic growth and poverty reduction, fiscal and debt sustainability, reducing external imbalances, and developing a sound financial system.

\section{Recent Developments and Outlook}

4. The pace of economic recovery thus far in $\mathbf{2 0 1 0}$ has been somewhat lower than projected at the time of the program approval, while inflation is higher. GDP growth was 3.1 percent in January-August 2010, driven by a recovery in services, industry, and construction, offset in part by an unprecedented decline in agricultural output due to bad weather conditions. Both trade and remittances have rebounded after a sharp decline last year, but the current account deficit, while declining, remains high. Fiscal performance has 
been strong in the first eight months of 2010 due to higher tax collections, including VAT and social contributions, and restrained spending. After falling to 5.7 percent in June (yearon-year), consumer prices increased to 9.6 percent in August mainly driven by a surge in food prices, notably for bread and fruit and vegetables. Banks remain well capitalized and liquid, and credit to the economy is picking up, notably in foreign currency.

5. In the short term, the economic outlook remains subdued. Growth in Russia and Europe-Armenia's largest trade partners-is likely to be sluggish in the near term. Due to the poor agricultural output and base effects from the resumption of growth in the last quarter of 2009, GDP growth could be slower in 2010 than projected earlier. On this basis, and mindful of downward risks, GDP is projected to grow at about 4 percent in 2010.

6. The medium-term outlook will continue to be challenging, and thus the pace of growth is uncertain and the economy is unlikely to return to pre-crisis growth rates. We expect GDP growth to rise to 4.6 percent in 2011, supported by strengthening domestic demand and a recovery of the agriculture sector. However, with external demand and remittances recovering only gradually, and FDI and private investment likely to remain weak, growth is expected to stay at about $4-4 \frac{1}{2}$ percent over 2012-13. Inflation is expected to revert to the CBA's target band during the second half of 2011. Public debt is also expected to drop to about 431/2 percent of GDP by 2013 under the newly approved debt management strategy and the medium-term expenditure framework. The current account deficit is expected to be reduced considerably.

\section{The Program for 2010 and 2011}

\section{A. Fiscal Policy and Debt Sustainability}

7. As the economy recovers, we are gradually unwinding the fiscal stimulus to ensure fiscal and debt sustainability. We remain committed to reducing our deficit to 4.8 percent of GDP in 2010 followed by a further reduction in 2011 to 3.9 percent of GDP. This reduction in the deficit is predicated on improvements in tax administration and limited increases in spending. With the improving economic activity and renewed efforts to strengthen tax administration, we expect the tax-to-GDP ratio to increase by about 0.5 percent per annum. During 2010-11, we will implement contingency measures if revenues underperform relative to projections under the program, including by adjusting spending as needed to achieve the deficit target. We will continue to adhere to our commitment to save at least half of any tax revenue over-performance above the current projections reflected in the EFF/ECF program. Over the medium term, given the need to free up some fiscal space because of a sharp increase in public debt during the recent crisis, our fiscal policy will aim at further reducing the deficit to about 2 percent of GDP by 2013.

8. In order to improve the transparency and planning of government operations, we re-introduced our medium-term expenditure framework (MTEF), suspended temporarily during the crisis. The MTEF, approved in August of this year, is geared at 
promoting growth, while placing public debt on a downward path and shifting spending toward social programs and investment in key sectors. With the economic crisis over, we intend to wind down our net lending program financed by the Russian loan, ensuring that such programs support small and medium-sized enterprises and exports. In addition, we have been consulting with our international partners on enhancing budgetary expenditure framework and the efficiency of spending.

9. Our efforts to strengthen debt management have materialized in the adoption of a new medium-term debt management strategy in July 2010. Under our program, we intend to keep the public and publicly guaranteed debt stock at about 50 percent of GDP in $2010-11$, and gradually reduce it over the medium term. We will also adhere to the average concessionality target as agreed under the program by strictly limiting nonconcessional borrowing to financially viable projects. We intend to strengthen our debt monitoring and planning capacity. We have recently adopted a time-bound action plan to implement the debt management strategy.

\section{B. Monetary and Exchange Rate Policy}

10. Monetary policy will continue to focus on price stability under our inflation targeting framework. We have kept our policy rate constant since May, communicating clearly to the market that the recent shock to prices is temporary and exogenous and that demand pressures remain subdued. We believe that second-round effects will not be large and inflation will gradually decline to within the target band by mid-2011. Nonetheless, we will continue monitoring price and output developments and adjust our monetary stance as needed.

11. We remain committed to a floating exchange rate. Our interventions in the foreign exchange market will continue to aim at smoothing large exchange rate movements, while not resisting fundamental trends. We also aim to continue guarding international reserves as a proven buffer from exogenous shocks. To avoid creating market perceptions of a return to maintaining an exchange rate target level or range, we will communicate clearly and act accordingly to ensure that market participants recognize the two-way risks in the foreign exchange market.

12. We will continue to strengthen the monetary transmission mechanism. We are striving to enhance the link between our policy rate and retail rates. To this end, we will manage liquidity actively to keep market interest rates close to the policy rate and, thus, advance the status of the policy rate as the market reference rate. To enhance further the interest rate channel, we are also considering initiatives to develop the market for dram instruments. We will standardize the pricing and settlement of treasury bills in line with international practice and facilitate transactions in the securities market. The law on mandatory third-party liability insurance passed in June 2010 and the law on private investment funds - currently with parliament—are expected to promote market development. 
13. We remain determined to reduce dollarization, given that it weakens the monetary transmission mechanism. An earlier regulation requiring a quarter of banks' reserves on foreign currency deposits to be maintained in drams has widened interest rate spreads between dram and foreign currency deposits. We have raised this proportion to one half in September aiming to trigger a further shift in the composition of deposits. In addition, the CBA and SRC are working on a plan to strengthen enforcement of foreign exchange regulations which require the use of drams in certain transactions and for listing the prices of goods and services. The draft law on restricting large cash transactions in the real sector is currently being reviewed by the parliament. We acknowledge that successful dedollarization will ultimately hinge on maintaining a track record of macroeconomic stability.

\section{Financial Sector Stability and Development}

14. We are committed to preserving the stability of the Armenian banking system and are promoting reforms to enhance its resilience and increase the efficiency of financial intermediation. Despite considerable improvement in recent years, private sector credit reached only 20 percent of GDP and residents are hoarding cash, including in foreign exchange. There is great scope for increasing financial access and deepening financial intermediation which would help improve the traction of monetary policy. We intend to undertake a full FSAP update in mid-2011, which would focus not only on financial stability issues, but also analyze the impediments to credit growth and financial intermediation.

\section{We continue our work on enhancing risk management and the supervisory}

framework to limit vulnerabilities in the financial sector. Recent prudential regulations have focused on ensuring proper management of foreign currency (FX) risk and FX-induced credit risk. In August 2010, the limits on net open foreign exchange positions were made symmetric at \pm 7 percent of bank capital. Prudential regulations on higher capital and provisioning requirements on foreign currency loans were issued to become effective in September 2010 and January 2011, respectively. In addition, we intend to require banks to pay extra attention to FX-induced credit risk assessment. Furthermore, in line with the Fund's technical assistance recommendations, we envisage to enhance our supervisory framework with a more forward-looking assessment of potential risks.

16. We continue strengthening our crisis preparedness and contingency planning. We have formalized the Committee for Financial Stability through a memorandum of understanding identifying actions and responsibilities of relevant institutions in various crisis events (Structural benchmark, September 2010). We are drafting a regulation requiring banks to set up their contingency plans for liquidity and solvency support (Structural benchmark, December 2010). We envisage a comprehensive contingency plan that would include stress tests designed by the banks themselves with an action plan on how to deal with these stress scenarios. 


\section{Structural Reforms}

\section{Fiscal Reforms}

\section{Fiscal reforms, notably enhancing revenue management, continue to be a} centerpiece of our program commitments. Our efforts are primarily focused on ensuring lasting improvements in tax administration, enhancing the integrity of the system, and achieving efficiency gains, while providing incentives for taxpayers to comply. We set up a tax appeals board under the government to deal with legal and procedural disputes of tax payers (Structural benchmark, September 2010). In case of a difference of opinion between the SRC and the appeals board, the decision of the Prime Minister will be binding and final for the SRC. Moreover, a government decree was issued stipulating that clarifications and interpretations of laws and legislative provisions on taxes, duties, and mandatory fees should be agreed in advance with the Ministry of Finance. We are finalizing the development of manuals in tourism, real estate, transport, and sectors using cash register machines for tax audits for usage starting January 2011 (Structural benchmark, December 2010). However, manuals for mining will be developed in 2011, as we continue to work with the World Bank on revising the mining code. Furthermore, continued progress has been achieved in (i) extending the e-filing system of tax returns to about 3,000 taxpayers, with all large taxpayers currently reporting electronically, and (ii) improving the quality of taxpayer services, including through the creation of a full scale taxpayer service center.

\section{Looking ahead, further measures are envisaged to modernize tax administration to} enhance revenue collection and secure a sustainable revenue base. Consistent with the earlier commitments for the near term we will:

- Overhaul the VAT refund processing system. We will adopt a government decree establishing a mechanism for implementing a fully functional risk management approach in VAT refund processing (Structural benchmark, December 2010), and ensure this approach is fully operational by June 2011 (Structural benchmark, June 2011).

- Increase the number of large taxpayers (LTPs) by about 100 by December 2010 with a view to ensuring that LTPs' contribution in terms of total domestic revenues is continuously increasing compared to the previous year.

- Simplify the reporting system by reducing the frequency of reporting to tax authorities and considerably streamlining tax forms for VAT, profit tax, and personal income tax (Structural benchmark, December 2010).

- Continue analyzing the systemic problems underlying the difficult issue of tax credits and implement measures to reduce the stock of tax credits by December 2010. 
19. Going forward, we will embark on a number of important reforms, including (i) structural and staffing reforms in tax inspectorates; (ii) strengthening the analytical and research capacity at the SRC and the Ministry of Finance; and (iii) launching a risk-based system for selection of taxpayers for audit, and implementing a comprehensive planning system for audits. We will develop a Three-Year Strategic Plan for Tax Administration (2012-15), outlining further reforms.

20. We are stepping up our efforts in the tax policy area. In particular, the presumptive taxation regime for petroleum products will be abolished and these products will be moved to the regular taxation regime starting January 2011. Moreover, we will gradually increase the presumptive payment for tobacco products with a view to harmonize the excise rates for imported and domestic tobacco over the course of 2011-13. Furthermore, we are committed to moving tobacco products from the presumptive to the regular taxation regime by 2014 , and have passed the law pertaining to these changes in October 2010. Finally, we have submitted legislation to parliament to allow transfer of certain activities identified in the Law on Presumptive Taxes to the general taxation system in September 2010.

21. We plan to introduce a funded pension pillar in 2014. The related legislative package has passed the first reading at the parliament. We will ensure that realistic estimates for the full costing of the reform plan are taken into account, including those associated with the planned increase in basic pensions.

\section{Other Structural Reforms}

22. We intend to update our Sustainable Development Program (SDP) by the time of the second review of the EFF/ECF program. The updated SDP will serve as the basis for our macroeconomic policy by providing an analytical background and ensuring a high participatory process of the civil society in economic management.

23. Corruption remains a serious impediment to development and growth and a major challenge for policy makers. In this area, we are committed to reversing the recent deterioration noted by several international indicators. Notably, a draft law has been submitted to parliament, which intends to address conflict of interest issues restricting public officials from engaging in commercial activities. We aim to strengthen the capacity of the competition commission in order to diminish monopolistic behavior in key sectors. We intend to take measures to strengthen competition and further reduce barriers to entry for new businesses, including a recent cabinet decree to create a one-stop shop for business registration.

24. A number of reforms are planned under the umbrella of the recently-launched negotiations for an EU Association Agreement with Armenia. We will revisit our regulations and practices regarding standardization, certification, intellectual property rights, competition rules, and public procurement procedures. The aim is to cut red tape and 
promote private investment and exports by bringing the above procedures and regulations in line with best international practice.

25. In the social policy area, we aim to reverse the recent increase in poverty and improve access to social services. Our reform efforts to improve the quality of education and health spending are expected to bear fruit through greater efficiency of spending. Also, we aim to gradually increase allocations for health spending which remain in particular low for Armenia's income level. In addition, we will continue our efforts to better target our antipoverty programs, as well as increasing their coverage. In collaboration with the World Bank, we will submit amendments to existing laws to enable the full functioning of an integrated system for the provision of social protection services (Structural benchmark, December 2011). We have also continued our progress toward achieving the Millennium Development Goals, as indicated by lower maternal mortality rates as well as an increase in life expectancy.

\section{Conclusion}

26. Given our strong program implementation, we request the completion of the first review of the EFF/ECF program and the associated disbursement of SDR 36.2 million.

We also request modification of the end-December performance criteria for net international reserves, net domestic assets, and the program fiscal balance. In addition, we request the establishment of performance criteria for end-June 2011. We will maintain a close policy dialogue with the Fund and stand ready to take additional measures, as appropriate, to ensure the achievement of the government social and economic objectives under the EFF/ECF program. We will continue to consult with the Fund on the adoption of measures, and in advance of revisions of the policies contained in the LOI, in accordance with the Fund's policies on such consultation. We will also provide the Fund with information it requests for monitoring progress during program implementation. The program's quantitative performance criteria and indicative targets, as per attached Technical Memorandum of Understanding and structural benchmarks are set out in Tables 1 and 2. We have cooperated with the IMF to update the safeguards assessment. The second review is expected to be completed on or after March 31, 2011 and will assess performance as of end-December 2010. The third review is expected to be completed on or after September 30, 2011. 
27. We authorize the IMF to publish this Letter of Intent and its attachments, as well as the accompanying staff report.

Very truly yours,

$$
\text { /s/ }
$$

Tigran Sargsyan

Prime Minister

Republic of Armenia

$/ \mathrm{s} /$

Tigran Davtyan Minister of Finance

Republic of Armenia
$/ \mathrm{s} /$

Artur Javadyan

Chairman of the Central Bank

Republic of Armenia 
Table 1. Armenia: Quantitative Targets for 2010-11 1/

(In billions of drams, at program exchange rates, unless otherwise specified)

\begin{tabular}{|c|c|c|c|c|c|c|c|c|c|c|c|c|c|c|c|c|}
\hline \multirow[b]{4}{*}{ Performance Criteria } & \multirow{3}{*}{$\begin{array}{r}2009 \\
\text { Dec. } \\
\text { Act. }\end{array}$} & \multicolumn{9}{|c|}{2010} & \multicolumn{6}{|c|}{2011} \\
\hline & & \multirow{2}{*}{$\begin{array}{l}\text { Mar. 21 } \\
\text { Act. }\end{array}$} & \multicolumn{3}{|c|}{ Jun. } & 2010 & \multicolumn{2}{|l|}{ Sep. 3/ } & \multicolumn{2}{|c|}{ Dec. } & \multicolumn{2}{|c|}{ Mar. 3/ } & \multicolumn{2}{|c|}{$\frac{2011}{\text { Jun. }}$} & Sep.31 & \multirow{2}{*}{$\begin{array}{c}\text { Dec. 3l } \\
\text { First Review }\end{array}$} \\
\hline & & & $\mathrm{EBS} / 10 / 119$ & Adj. Prog. & Act. & $\mathrm{EBS} / 10 / 119$ & Adj. Prog. & Prel. & $\mathrm{EBS} / 10 / 119$ & First Review & EBS/10/119 & First Review & $\mathrm{EBS} / 10 / 119$ & First Review & w First Review & \\
\hline & & & & & & & & & & & & & & & & \\
\hline Net official international reserves (floor, in millions of U.S. dollars) & 1,019 & 895 & 824 & 824 & 912 & 855 & 787 & 929 & 829 & 808 & 856 & 831 & 793 & 78 & 801 & 747 \\
\hline Net domestic assets of the CBA (ceiling) & -12 & -28 & 38 & 38 & -16 & 46 & 74 & -9 & 85 & 125 & 51 & 99 & 95 & 12 & 138 & 190 \\
\hline Program fiscal balance (floor) $4 /$ & -355 & 3 & -75 & -75 & -24 & -92 & -92 & -76 & -133 & -140 & -36 & -34 & -88 & -5 & -90 & -112 \\
\hline External public debt arrears (continuous criterion) & 0 & 0 & 0 & 0 & 0 & 0 & 0 & 0 & 0 & 0 & 0 & 0 & 0 & & 0 & 0 \\
\hline Indicative Targets & & & & & & & & & & & & & & & & \\
\hline Reserve money (ceiling) & & 465 & 503 & 508 & 435 & 534 & 539 & 438 & 575 & 540 & 541 & 515 & 570 & 53 & 555 & 596 \\
\hline Average concessionality of newly disbursed debt (floor, in percent) & & & 30 & 30 & 31 & 30 & 30 & 30 & 30 & 30 & 30 & 30 & 30 & & 30 & 30 \\
\hline Social spending of the government (floor) $5 /$ & & 7 & 15 & 15 & 15 & 23 & 23 & 23 & 31 & 31 & 9 & 9 & 18 & & 27 & 36 \\
\hline
\end{tabular}

Sources: Armenian authorities; and Fund staff estimates.

1/ All items as defined in the TMU.
$2 /$ The net official international reserves balance excludes the total IMF disbursement received by Armenia on March 31

Indicative target.

4/ Bdicaw-the-line overall balance excluding net lending until 2009. Below-the-line overall balance excluding net lending and project financing from 2010. 
Table 2. Armenia: Structural Benchmarks Under the EFF/ECF Arrangement

\begin{tabular}{|c|c|c|c|}
\hline Item & Measure & $\begin{array}{l}\text { Proposed Time Frame } \\
\text { (End of Period) }\end{array}$ & Outcome \\
\hline \multicolumn{4}{|c|}{$\underline{\text { Tax administration }}$} \\
\hline 1 & $\begin{array}{l}\text { Issue a government decree stipulating that clarifications and interpretations of } \\
\text { laws, regulations, and procedures on taxes, duties, and mandatory fees } \\
\text { should be approved by the Ministry of Finance. These clarifications and } \\
\text { interpretations will become normative acts to be published and applied } \\
\text { consistently across all taxpayers effective September } 1,2010 \text {. }\end{array}$ & July 2010 & Met \\
\hline 2 & $\begin{array}{l}\text { Set up a tax appeals board under the government to deal with legal and } \\
\text { procedural disputes of taxpayers. }\end{array}$ & September 2010 & Met with delay \\
\hline 3 & $\begin{array}{l}\text { Develop manuals in tourism, real estate, transport, and sectors using cash } \\
\text { register machines for tax audits for usage starting January } 2011 .\end{array}$ & December 2010 & \\
\hline 4 & $\begin{array}{l}\text { Simplify the reporting system by reducing the frequency of reporting to tax } \\
\text { authorities and considerably streamline tax forms for VAT, profits tax, and } \\
\text { personal income tax. }\end{array}$ & December 2010 & \\
\hline 5 & $\begin{array}{l}\text { Adopt a government decree establishing a mechanism for implementing a fully } \\
\text { functional risk management approach in VAT refund processing. }\end{array}$ & December 2010 & \\
\hline 6 & $\begin{array}{l}\text { Implement a fully functional risk-based management approach in VAT refund } \\
\text { processing. }\end{array}$ & June 2011 & \\
\hline \multicolumn{4}{|c|}{ Social policy } \\
\hline 7 & $\begin{array}{l}\text { Submit amendments to existing laws to parliament to enable the full } \\
\text { functioning of an integrated system for the provision of social protection } \\
\text { services. }\end{array}$ & December 2011 & \\
\hline \multicolumn{4}{|c|}{ Fiscal and debt sustainability } \\
\hline 8 & $\begin{array}{l}\text { Approve a medium-term expenditure framework (2011-13), including a } \\
\text { medium-term debt management strategy. }\end{array}$ & August 2010 & Met \\
\hline \multicolumn{4}{|c|}{ Financial sector } \\
\hline 9 & $\begin{array}{l}\text { Issue prudential regulations to specifically address currency-induced credit } \\
\text { risk, including increased loan-loss provisioning requirements and higher risk } \\
\text { weights in capital requirements for foreign currency loans. }\end{array}$ & June 2010 & Met \\
\hline 10 & $\begin{array}{l}\text { Formalize the Committee for Financial Stability in an MOU to set the } \\
\text { modalities for main policy makers to coordinate their policies and responses in } \\
\text { case of an imminent critical situation in the banking sector. }\end{array}$ & September 2010 & Met \\
\hline 11 & $\begin{array}{l}\text { Issue prudential regulation requiring banks to prepare their contingency plans } \\
\text { for liquidity and solvency support. }\end{array}$ & December 2010 & \\
\hline
\end{tabular}




\section{AtTaChment II. Armenia: TeChniCAl Memorandum of Understanding}

1. This memorandum sets out the understandings between the Armenian authorities and the IMF staff regarding the definition of performance criteria and indicative targets, their adjusters, and data reporting requirements for the three-year EFF/ECF Arrangement as per the Letter of Intent dated November 11, 2010 (LOI).

2. For program monitoring purposes, all foreign currency-related assets, liabilities, and flows in the monetary accounts will be evaluated at program exchange rates. The program exchange rate of the Armenian dram to the U.S. dollar is set at dram 385 per one U.S. dollar. The cross-rates for other foreign currencies are provided in Table 1.

\section{Quantitative Targets}

3. The program sets performance criteria and indicative targets for defined test dates (see Table 1 in the November 2010 LOI ). The program sets the following performance criteria:

- Floor on the net official international reserves (NIR) of the Central Bank of Armenia (CBA);

- Ceiling on the net domestic assets (NDA) of the CBA;

- Ceiling on external public debt arrears (continuous); and

- Floor on the program fiscal balance;

The program sets the following indicative targets:

- Ceiling on reserve money;

- Floor on average concessionality of new debt (quarterly on a disbursement basis); and

- Floor on social spending of the government.

4. The net official international reserves (stock) of the Central Bank of Armenia (CBA) will be calculated as the difference between total gross official international reserves and gross official reserve liabilities.

- Gross official international reserves are defined as the CBA's holdings of monetary gold (excluding amounts pledged as collateral), holdings of Special Drawing Rights (SDRs), including the August 28, 2009 General allocation and the September 9, 2009 Special allocation, the country's reserve position at the IMF, and holdings of convertible currencies in cash or in nonresident financial institutions (deposits, securities, or other financial instruments). Gross reserves held in the form of 
securities and other financial instruments are marked to market. Excluded from gross reserves are the balance on the government's Special Privatization Account (SPA), capital subscriptions in foreign financial institutions and illiquid foreign assets, any assets that are pledged, collateralized, or otherwise encumbered, claims on residents, claims in foreign exchange arising from derivatives in foreign currencies vis-à-vis domestic currency (such as futures, forwards, swaps, and options), precious metals other than gold, assets in nonconvertible currencies, and illiquid assets.

- Official reserve liabilities shall be defined as the total outstanding liabilities of the government and the CBA to the IMF and convertible currency liabilities of the CBA to nonresidents with an original maturity of up to and including one year, as well as commitments to sell foreign exchange arising from derivatives (such as futures, forwards, swaps, and options).

NIR is monitored in U.S. dollars, and, for program monitoring purposes, assets and liabilities in currencies other than the U.S. dollar shall be converted into dollar-equivalent values using the exchange rates as specified in Table 1.

5. Reserve money is defined as the sum of currency issued, required and excess reserves, and current and time deposit accounts of certain resident agents. Liquidity absorbing transactions under reverse repurchase agreements, the CBA's deposit facility, foreign currency swaps, and securities issued by the CBA are excluded from the reserve money definition. The ceiling will be considered as met if the outcome is within AMD 5 billion of the indicative target set in Table 1 attached to the Letter of Intent dated November 11, 2010.

6. The net domestic assets are defined as reserve money minus NIR, minus reserve money denominated in foreign currencies, plus medium and long-term liabilities (i.e. liabilities with a maturity of one year or more) of the CBA, plus the balance of outstanding Fund purchases credited to the government account at the CBA. NDA is composed of net CBA credit to the general government; outstanding credit to domestic banks by the CBA (including overdrafts) minus liabilities not included in reserve money and other items net. External public debt arrears are defined as all unpaid debt-service obligations (i.e., payments of principal and interest) arising in respect of public sector loans contracted or guaranteed, including unpaid penalties or interest charges associated with these obligations that are overdue beyond 30 days after the due date. ${ }^{1}$ The ceiling on external payment arrears is set at zero.

\footnotetext{
${ }^{1}$ The public sector is defined following the Government Financial Statistics Manual (GFS 2001) and System of National Accounts (1993 SNA). It includes the general government and nonfinancial public enterprises (as defined in paragraph 11).
} 
7. The program fiscal balance is cumulative from the beginning of the fiscal year and is measured from the financing side as the negative of the sum of net domestic banking system credit to the central government, net domestic nonbank financing, and net external financing to the central government (excluding net project financing). Should a general subsidy be introduced off-budget, the overall balance will be measured including the subsidy as part of government spending.

- Net banking system credit to the central government equals the change during the period of net credit to the central government.

- Net nonbank financing equals the sum of: (1) the change during the period of outstanding treasury bills and bonds to nonbanks (including accrued interest for treasury bills and excluding accrued interest for treasury bonds); ${ }^{2}$ (2) any other disbursement or transaction that increases nonbanks' claims on the central government plus withdrawals from the special privatization account or the treasury sub-account containing privatization proceeds in drams, less amortization paid by the central government to private resident nonbank agents.

- Net external financing equals total debt-increasing disbursements from nonresidents to the central government (including Fund net purchases credited directly to the government accounts at the CBA) less total amortization from the central government to non-residents. All foreign currency-denominated transactions are recorded in drams using the prevailing exchange rate at the time of the transaction.

8. Those project implementation units, which carry out projects financed by the U.S.based Lincy Foundation, or other budget-related project implementation units maintain accounts at the CBA. The grants are recorded in the fiscal accounts as external grants on the revenue side and as foreign-financed capital expenditure on the expenditure side. In addition, any loans to finance investments and that are intermediated through the banking system are recorded in the financial accounts as a financing item below the line and are thus excluded from net lending.

9. Foreign currency proceeds from selling enterprises are deposited into the Special Privatization Account (SPA). The account is held at the CBA and the proceeds are invested abroad together with the CBA's international reserves. These proceeds are included in the definition of the monetary accounts of the CBA as part of net foreign assets with a counter entry in other items net. Any budgeted withdrawal from the SPA will be accounted for as privatization proceeds used to finance the budget and will be recorded below the line. Any unanticipated withdrawal from the SPA will be recorded below the line as privatization

\footnotetext{
${ }^{2}$ Domestic nonbank holdings of treasury bills and treasury bonds are defined as total outstanding treasury bills and bonds less holdings by the banking system and the State Fund for Social Insurance.
} 
receipts; these withdrawals, however, will be replenished during the same fiscal year. Domestic currency proceeds from selling enterprises to residents are deposited in a subaccount of the treasury single account.

10. The program sets a floor of 30 percent on average concessionality of new debt on a disbursement basis on debt with nonresidents with original maturities of one year or more contracted and guaranteed by the public sector during the period 2010 .

- The monitoring of the average concessionality target is done on a disbursement-bydisbursement basis and consistent with the methodology used in the DSA to calculate the original average concessionality target. In particular, the discount rate and the exchange rates used in the DSA should be used for the monitoring.

- The public sector comprises the general government, the central bank, and nonfinancial public enterprises (enterprises and agencies in which the government holds a controlling stake - typically owns more than 50 percent of the shares, but which are not consolidated in the budget).

- For program purposes, the guarantee of a debt arises from any explicit legal obligation of the public sector to service a debt in the event of nonpayment by the debtor (involving payments in cash or in kind), or from any implicit legal or contractual obligation of the public sector to finance partially or in full any shortfall incurred by the debtor.

11. The program sets a floor on social spending of the government. For the purposes of the program, social spending of the government is defined as 100 percent of the budgeted amount of the family benefit program and lump-sum financial aid.

\section{Adjustors}

12. The quantitative performance criteria and indicative targets under the program are subject to the following adjusters, calculated, where relevant, using program exchange rates:

- Changes in reserve requirements: The ceiling on the NDA of the CBA and the ceiling on reserve money will be adjusted downward (upward) by the amount of banks' reserves freed (seized) by any reduction (increase) of the reserve requirement ratio on both domestic currency and foreign currency deposits relative to the baseline assumption as per the following formula: $\triangle \mathrm{NDA}=\Delta \mathrm{rB}$, where $\mathrm{B}$ denotes the level of the deposits subject to reserve requirements in the initial definition and $\Delta \mathrm{r}$ is the change in the reserve requirement ratio.

- KfW and World Bank loan disbursements: the ceiling on the NDA of the CBA will be adjusted upward (downward) by the full amount of any excess (shortfall) of disbursements from the KfW and World Bank loans directed at SME financing 
compared to programmed amounts (Table 2). The floor on NIR will be adjusted upward (downward) by the cumulative amount of any excess (shortfall) of these disbursements compared to program amounts.

- Budget support grants to the public sector are defined as grants received by the general government for direct budget support from external donors and not related to project financing.

- External financing to the public sector is defined as disbursements of loans from bilateral and multilateral donors for budget support and Fund purchases credited directly to the government accounts at the CBA, with the exception of the KfW and World Bank disbursements mentioned above:

- The floor on NIR will be adjusted upward (downward) by the cumulative amount of any excess (shortfall) of external financing or EU grants in the form of budget support (excluding Fund disbursements to the government) compared to program amounts (Table 3). The floor on NIR will be adjusted downward for any external public debt amortization amounts in excess of program amounts.

- The ceiling on NDA will be adjusted downward by the amount of any excess of external financing or EU grants in the form of budget support compared to program amounts.

- The floor on the program fiscal balance on a cash basis will be adjusted upward (downward) by the cumulative total amount of the EU budget grants received in excess (to account for a shortfall) of the program amounts (Table 3). 


\section{Data Reporting}

13. The government will provide the IMF the information specified in the following table.

\begin{tabular}{|c|c|c|c|c|}
\hline $\begin{array}{l}\text { Reporting } \\
\text { Agency }\end{array}$ & Type of Data & Description of Data & Frequency & Timing \\
\hline \multirow[t]{11}{*}{$\mathrm{CBA}^{3}$} & CBA balance sheet & Summary & Daily & $\begin{array}{l}\text { The following } \\
\text { working day }\end{array}$ \\
\hline & CBA balance sheet & $\begin{array}{l}\text { Summary at program exchange rates; } \\
\text { and by chart of accounts at actual } \\
\text { official exchange rates }\end{array}$ & Monthly & $\begin{array}{l}\text { Within seven days } \\
\text { of the end of each } \\
\text { month }\end{array}$ \\
\hline & Monetary survey & $\begin{array}{l}\text { Summary banking system balance sheet } \\
\text { for the central bank at both program } \\
\text { exchange rates and by chart of accounts } \\
\text { at actual official exchange rates; the } \\
\text { consolidated balance sheet of } \\
\text { commercial banks by chart of accounts } \\
\text { at actual official exchange rates }\end{array}$ & Monthly & $\begin{array}{l}\text { Within } 25 \text { days of } \\
\text { the end of each } \\
\text { month }\end{array}$ \\
\hline & International reserves & $\begin{array}{l}\text { By chart of accounts; at (i) program } \\
\text { exchange rates; and (ii) at actual } \\
\text { official exchange rates }\end{array}$ & Daily & $\begin{array}{l}\text { The following } \\
\text { working day }\end{array}$ \\
\hline & $\begin{array}{l}\text { Foreign exchange } \\
\text { market }\end{array}$ & $\begin{array}{l}\text { Official exchange rates (buying and } \\
\text { selling); daily trade volume, and } \\
\text { weighted average exchange rate of the } \\
\text { interbank, intrabank and Nasdaq-OMX }\end{array}$ & Daily & Within 1 day \\
\hline & $\begin{array}{l}\text { Foreign exchange } \\
\text { market }\end{array}$ & $\begin{array}{l}\text { CBA foreign exchange operations, } \\
\text { (exchange rate, volume, counterpart) }\end{array}$ & Daily & Within 1 day \\
\hline & $\begin{array}{l}\text { Foreign exchange } \\
\text { market }\end{array}$ & Exchange rate (buying and selling) & Daily & Within 1 day \\
\hline & Interest rates & Refinance rate & $\begin{array}{l}\text { At least } \\
\text { monthly }\end{array}$ & $\begin{array}{l}\text { Within } 1 \text { days of } \\
\text { the CBA Board } \\
\text { decision }\end{array}$ \\
\hline & $\begin{array}{l}\text { Interbank money } \\
\text { market }\end{array}$ & $\begin{array}{l}\text { Daily interbank repo volume and } \\
\text { interest rate and number of trades }\end{array}$ & Daily & Within 1 day \\
\hline & CBA operations & $\begin{array}{l}\text { Repo (reverse repo) operations, open } \\
\text { market operations, Lombard credits, } \\
\text { deposit facility, and foreign exchange } \\
\text { swaps (volumes, maturity, yields, } \\
\text { exchange rates) }\end{array}$ & Daily & Within 1 day \\
\hline & Bank liquidity & $\begin{array}{l}\text { Reserves and excess reserves, by } \\
\text { currency }\end{array}$ & Monthly & $\begin{array}{l}\text { Within } 15 \text { days of } \\
\text { the end of each } \\
\text { reference period }\end{array}$ \\
\hline
\end{tabular}

${ }^{3}$ As defined in CBA resolution No. 201 (December 6, 1999). 


\begin{tabular}{|c|c|c|c|c|}
\hline & $\begin{array}{l}\text { Interest rates and flows } \\
\text { of the funds attracted } \\
\text { and allocated by } \\
\text { commercial banks }\end{array}$ & By currency and maturity & Weekly & $\begin{array}{l}\text { Last working day } \\
\text { of the week }\end{array}$ \\
\hline & $\begin{array}{l}\text { T-bill and coupon bond } \\
\text { financing, CBA } \\
\text { securities }\end{array}$ & $\begin{array}{l}\text { Auction data: date, original and } \\
\text { remaining maturities, issuance volume, } \\
\text { allocation, average yield and coupon } \\
\text { yield (if available) }\end{array}$ & Weekly & $\begin{array}{l}\text { Last working day } \\
\text { of the week }\end{array}$ \\
\hline & Banking data & $\begin{array}{l}\text { Sectoral distribution of loans and } \\
\text { deposits; dollarization of loans and } \\
\text { deposits; loan maturities; interbank } \\
\text { rate, by volume and maturity; T-bill } \\
\text { rate, bond yield; deposit and lending } \\
\text { rates, by maturity; monthly weighted } \\
\text { average interest rate on government } \\
\text { bonds }\end{array}$ & Monthly & $\begin{array}{l}\text { Within } 25 \text { days of } \\
\text { the end of each } \\
\text { month }\end{array}$ \\
\hline & Banking indicators & $\begin{array}{l}\text { Capital adequacy; asset composition } \\
\text { and quality; profitability; liquidity; } \\
\text { open FX positions; and compliance } \\
\text { with prudential norms }\end{array}$ & Monthly & $\begin{array}{l}\text { Within } 30 \text { days of } \\
\text { the end of each } \\
\text { month }\end{array}$ \\
\hline & $\begin{array}{l}\text { Banking sector stress } \\
\text { tests }\end{array}$ & $\begin{array}{l}\text { Results of stress tests on exchange rate, } \\
\text { liquidity, and credit risk }\end{array}$ & Monthly & $\begin{array}{l}\text { Within } 30 \text { days of } \\
\text { the end of each } \\
\text { month }\end{array}$ \\
\hline & CPI & Index of core inflation & Monthly & $\begin{array}{l}\text { Within } 21 \text { days of } \\
\text { the end of each } \\
\text { month }\end{array}$ \\
\hline & Transfers & $\begin{array}{l}\text { Non-commercial transfers of } \\
\text { individuals }\end{array}$ & Monthly & $\begin{array}{l}\text { Within } 30 \text { of the } \\
\text { end of each month }\end{array}$ \\
\hline & Other monetary data & IFS format & Monthly & $\begin{array}{l}\text { Within } 45 \text { days of } \\
\text { the end of each } \\
\text { month }\end{array}$ \\
\hline \multirow{3}{*}{$\begin{array}{l}\text { Ministry of } \\
\text { Finance } \\
(\mathrm{MOF})\end{array}$} & $\begin{array}{l}\text { T-bill and coupon bond } \\
\text { financing }\end{array}$ & $\begin{array}{l}\text { By holders, i.e., CBA, resident banks, } \\
\text { resident nonbanks, and nonresidents }\end{array}$ & Monthly & $\begin{array}{l}\text { Within } 7 \text { days of } \\
\text { each month }\end{array}$ \\
\hline & External debt & $\begin{array}{l}\text { Disbursements and stock of outstanding } \\
\text { short-term and contracting or } \\
\text { guaranteeing and outstanding stock of } \\
\text { medium-and long-term external debt of } \\
\text { the government, the CBA, and state- } \\
\text { owned companies (by company); any } \\
\text { stock of arrears on external debt service } \\
\text { and outstanding stock of government } \\
\text { guarantees and external arrears }\end{array}$ & Monthly & $\begin{array}{l}\text { Within } 21 \text { days of } \\
\text { the end of each } \\
\text { month . } \\
\text { For project } \\
\text { implementation } \\
\text { units, within } 21 \\
\text { days of the end of } \\
\text { each month } \\
\text { (preliminary data) } \\
\text { and within } 45 \text { days } \\
\text { of the end of each } \\
\text { month (final data) }\end{array}$ \\
\hline & Revenue collection & $\begin{array}{l}\text { Total revenue collected separately by } \\
\text { the tax administration and customs } \\
\text { administration, including revenue by } \\
\text { individual tax, and social contributions }\end{array}$ & Monthly & $\begin{array}{l}\text { Within } 7 \text { days of } \\
\text { the end of each } \\
\text { month }\end{array}$ \\
\hline
\end{tabular}




\begin{tabular}{|c|c|c|c|c|}
\hline & $\begin{array}{l}\text { Domestic expenditure } \\
\text { arrears }\end{array}$ & $\begin{array}{l}\text { All unpaid claims outstanding at the } \\
\text { end of the month which includes } \\
\text { wages, social contributions (including } \\
\text { for pensions), family allowances, and } \\
\text { amortization and domestic interest } \\
\text { payments }\end{array}$ & Monthly & $\begin{array}{l}\text { Within } 45 \text { days of } \\
\text { the end of each } \\
\text { month for } \\
\text { government arrears }\end{array}$ \\
\hline & Privatization receipts & $\begin{array}{l}\text { Balance on the SPA; gross inflows into } \\
\text { and outflows from the SPA during the } \\
\text { month, specifying the nature of each } \\
\text { transaction }\end{array}$ & Monthly & $\begin{array}{l}\text { Within } 7 \text { days of } \\
\text { the end of each } \\
\text { month }\end{array}$ \\
\hline & $\begin{array}{l}\text { Treasury single account } \\
\text { (TSA) }\end{array}$ & $\begin{array}{l}\text { Detailed breakdown of central treasury } \\
\text { account, including deposits at the } \\
\text { central treasury, community budgets, } \\
\text { off budget account, monetization } \\
\text { account, state budget account and the } \\
\text { Republic correspondent account-- } \\
\text { flows during the month and end of } \\
\text { month stocks. }\end{array}$ & Monthly & $\begin{array}{l}\text { Within } 7 \text { days of } \\
\text { the end of each } \\
\text { month }\end{array}$ \\
\hline & $\begin{array}{l}\text { Consolidated central } \\
\text { government }\end{array}$ & State budget & Monthly & $\begin{array}{l}\text { Within } 30 \text { days of } \\
\text { the end of each } \\
\text { month }\end{array}$ \\
\hline & $\begin{array}{l}\text { Consolidated general } \\
\text { government }\end{array}$ & $\begin{array}{l}\text { Central and local governments, and } \\
\text { Non-Commercial Enterprises that } \\
\text { belong within the general government } \\
\text { (NCEs) }\end{array}$ & Quarterly & $\begin{array}{l}\text { Within } 60 \text { days of } \\
\text { the end of each } \\
\text { quarter }\end{array}$ \\
\hline & $\begin{array}{l}\text { Consolidated general } \\
\text { government }\end{array}$ & $\begin{array}{l}\text { Central and local governments, and } \\
\text { NCEs that belong within the general } \\
\text { government }\end{array}$ & Annual & $\begin{array}{l}\text { Within } 180 \text { days of } \\
\text { the end of each } \\
\text { year }\end{array}$ \\
\hline & Budget execution & $\begin{array}{l}\text { All cash receipts, cash expenditures, } \\
\text { including domestic and external debt- } \\
\text { service payments, external and } \\
\text { domestic borrowing operations, and } \\
\text { inflow of grants to the central } \\
\text { government; expenditure data will be } \\
\text { provided according to both economic } \\
\text { and functional classifications, } \\
\text { consistent with the GFSM2001 } \\
\text { methodology }\end{array}$ & Monthly & $\begin{array}{l}\text { Within one month } \\
\text { following the end } \\
\text { of each quarter. }\end{array}$ \\
\hline NSS & Balance of payments & Detailed export and import data & Monthly & $\begin{array}{l}\text { Within } 28 \text { days of } \\
\text { the end of each } \\
\text { month }\end{array}$ \\
\hline & & Detailed export and import data & Quarterly & $\begin{array}{l}\text { Within } 45 \text { days of } \\
\text { the end of each } \\
\text { quarter }\end{array}$ \\
\hline & & Detailed balance of payments data & quarterly & $\begin{array}{l}\text { Within } 60 \text { days of } \\
\text { the end of each } \\
\text { quarter }\end{array}$ \\
\hline & CPI & By category & Monthly & $\begin{array}{l}\text { Within } 5 \text { days of } \\
\text { the end of each } \\
\text { month }\end{array}$ \\
\hline
\end{tabular}




\begin{tabular}{|c|c|c|c|c|}
\hline \multirow[t]{6}{*}{$\begin{array}{l}\text { State } \\
\text { Revenue } \\
\text { Committee }\end{array}$} & \multirow[t]{2}{*}{ Tax arrears } & By type of tax & Monthly & $\begin{array}{l}\text { Within } 30 \text { days of } \\
\text { the end of each } \\
\text { quarter (monthly } \\
\text { data provided on a } \\
\text { quarterly basis) }\end{array}$ \\
\hline & & $\begin{array}{l}\text { For or the } 30 \text { largest debtors and for all } \\
\text { major companies in the energy, water, } \\
\text { and irrigation sectors }\end{array}$ & Quarterly & $\begin{array}{l}\text { Within } 30 \text { days of } \\
\text { the end of each } \\
\text { quarter }\end{array}$ \\
\hline & Tax credits & $\begin{array}{l}\text { Detailed data, by type of tax, of } \\
\text { outstanding tax credits for all types of } \\
\text { tax revenues }\end{array}$ & Monthly & $\begin{array}{l}\text { Within } 45 \text { days of } \\
\text { the end of each } \\
\text { month (monthly } \\
\text { data provided on a } \\
\text { quarterly basis) }\end{array}$ \\
\hline & $\begin{array}{l}\text { VAT refund claims in } \\
\text { arrears }\end{array}$ & $\begin{array}{l}\text { Detailed data on VAT refunds in } \\
\text { arrears which include all outstanding } \\
\text { VAT refunds that have not been } \\
\text { accepted (and refunded), or offset (in } \\
\text { full or in part), or rejected (in full or in } \\
\text { part) after the } 90 \text { day statutory } \\
\text { processing period. Number of refund } \\
\text { applications processed per month. }\end{array}$ & Monthly & $\begin{array}{l}\text { Within } 45 \text { days of } \\
\text { the end of each } \\
\text { month (monthly } \\
\text { data provided on a } \\
\text { quarterly basis) }\end{array}$ \\
\hline & Large taxpayers & $\begin{array}{l}\text { Data on the number of taxpayers and } \\
\text { amount of taxes managed by the large } \\
\text { tax inspectorate }\end{array}$ & Monthly & $\begin{array}{l}\text { Within } 45 \text { days } \\
\text { after the end of } \\
\text { each month }\end{array}$ \\
\hline & Import data & $\begin{array}{l}\text { 1. Total value of recorded imports, } \\
\text { breaking out raw diamond imports; } \\
\text { 2. Total value of non-duty free } \\
\text { recorded imports; } \\
\text { 3. Number of total transactions } \\
\text { involving recorded imports; } \\
\text { 4. Number of total transactions } \\
\text { involving non-duty free recorded } \\
\text { imports } \\
\text { 5. Value of recorded imports where } \\
\text { customs value was assessed using } \\
\text { transaction prices, breaking out raw } \\
\text { diamond imports; } \\
6 . \text { Value of non-duty free recorded } \\
\text { imports where customs value was } \\
\text { assessed using transaction prices; } \\
\text { 7. Number of transactions involving } \\
\text { recorded imports where customs value } \\
\text { was assessed using transaction prices; } \\
8 . \text { Number of transactions involving } \\
\text { non duty free recorded imports where } \\
\text { customs value was assessed using } \\
\text { transaction prices }\end{array}$ & Quarterly & $\begin{array}{l}\text { Within } 30 \text { days of } \\
\text { the end of each } \\
\text { quarter }\end{array}$ \\
\hline
\end{tabular}


Table 1. Armenia: (Program) Exchange Rates of the CBA (As of December 31, 2008 in U.S. dollars per currency rates)

\begin{tabular}{lcc}
\hline & $\begin{array}{c}\text { Drams } \\
\text { Per } \\
\text { Currency }\end{array}$ & $\begin{array}{c}\text { Dollars } \\
\text { Per } \\
\text { Currency }\end{array}$ \\
\hline Australian dollar & 266.57 & 0.6924 \\
Canadian dollar & 313.39 & 0.8140 \\
Swiss franc & 365.58 & 0.9496 \\
Danish krone & 73.26 & 0.1903 \\
Euro & 546.00 & 1.4182 \\
Pound sterling & 558.68 & 1.4511 \\
Japanese yen & 4.27 & 0.0111 \\
Norwegian krone & 55.20 & 0.1434 \\
Russian ruble & 13.13 & 0.0341 \\
Swedish krone & 18.21 & 0.0473 \\
SDR & 593.00 & 1.5403 \\
\hline
\end{tabular}

Table 2. Armenia: KFW and IBRD SME Loan Disbursements, 2010-11 1/ (In millions of U.S. dollars)

\begin{tabular}{|c|c|c|c|c|c|c|c|c|c|c|c|c|c|}
\hline Dec-09 & Mar-10 & Jun- & & Sep- & & Dec- & & Mar- & & Jun- & & Sep-11 & Dec-11 \\
\hline Actual & Actual & EBS/10/119 & Actual & EBS/10/119 & Actual & EBS/10/119 & Prog. & EBS/10/119 & Prog. & $\mathrm{EBS} / 10 / 119$ & Prog. & Prog. & Prog. \\
\hline 33.8 & 0.0 & 0.0 & 0.0 & 2.0 & 5.0 & 27.8 & 29.9 & 2.0 & 2.0 & 4.0 & 4.0 & 6.0 & 8.0 \\
\hline
\end{tabular}

Table 3. Armenia: External Financing to the Public Sector in 2010-11 $1 /$ (In millions of U.S. dollars)

\begin{tabular}{|c|c|c|c|c|c|c|c|c|c|c|c|c|c|c|}
\hline & \multirow{2}{*}{$\frac{\text { Dec-09 }}{\text { Actual 2/ }}$} & \multirow{2}{*}{$\frac{\text { Mar-10 }}{\text { Actual }}$} & \multicolumn{2}{|c|}{ Jun-10 } & \multicolumn{2}{|l|}{ Sep-10 } & \multicolumn{2}{|c|}{ Dec-10 } & \multicolumn{2}{|c|}{ Mar-11 } & \multicolumn{2}{|c|}{ Jun-11 } & \multirow{2}{*}{$\frac{\text { Sep-11 }}{\text { Prog. }}$} & \multirow{2}{*}{$\frac{\text { Dec-11 }}{\text { Prog. }}$} \\
\hline & & & Prog. & Actual & EBS/10/119 & Prel. & EBS/10/119 & Prog. & EBS/10/119 & Prog. & EBS/10/119 & Prog. & & \\
\hline Project financing & 261 & 62 & 95 & 106 & 143 & 148 & 188 & 187 & 4 & 14 & 43 & 61 & 95 & 159 \\
\hline Budget financing & 304 & 0 & 38 & 38 & 114 & 67 & 163 & 116 & 57 & 56 & 86 & 85 & 131 & 160 \\
\hline EU grant & $\ldots$ & $\ldots$ & $\ldots$ & $\cdots$ & 23 & 0 & 23 & 0 & 23 & 25 & 23 & 25 & 50 & 50 \\
\hline Total & 565 & 62 & 133 & 144 & 281 & 216 & 374 & 303 & 84 & 95 & 152 & 171 & 275 & 368 \\
\hline
\end{tabular}

$1 /$ Cumulative from the end of the previous year, excluding non-EU grants. 2/ Excluding Russia loan in the amount of 500 million U.S. dollars. 


\section{Guidelines on the Indicative Benchmark with Respect to the Definition of External Debt}

For program purposes, the definition of debt is set out in Executive Board Decision No. 12274, Point 9, as revised on August 31, 2009 (Decision No. 14416-(09/91)).

(a) For the purpose of this guideline, the term "debt" will be understood to mean a current, i.e., not contingent, liability, created under a contractual arrangement through the provision of value in the form of assets (including currency) or services, and which requires the obligor to make one or more payments in the form of assets (including currency) or services, at some future point(s) in time; these payments will discharge the principal and/or interest liabilities incurred under the contract. Debts can take a number of forms, the primary ones being as follows:

(i) loans, that is, advances of money to obligor by the lender made on the basis of an undertaking that the obligor will repay the funds in the future (including deposits, bonds, debentures, commercial loans, and buyers' credits) and temporary exchanges of assets that are equivalent to fully collateralized loans under which the obligor is required to repay the funds, and usually pay interest, by repurchasing the collateral from the buyer in the future (such as repurchase agreements and official swap arrangements);

(ii) suppliers' credits, that is, contracts where the supplier permits the obligor to defer payments until some time after the date on which the goods are delivered or services are provided; and

(iii) leases, that is, arrangements under which property is provided which the lessee has the right to use for one or more specified period(s) of time that are usually shorter than the total expected service life of the property, while the lessor retains the title to the property. For the purpose of the guideline, the debt is the present value (at the inception of the lease) of all lease payments expected to be made during the period of the agreement excluding those payments that cover the operation, repair or maintenance of the property.

(b) Under the definition of debt set out in point 9 (a) above, arrears, penalties, and judicially awarded damages arising from the failure to make payment under a contractual obligation that constitutes debt are debt. Failure to make payment on an obligation that is not considered debt under this definition (for example, payment on delivery) will not give rise to debt. 


\title{
INTERNATIONAL MONETARY FUND
}

\author{
REPUBLIC OF ARMENIA
}

\section{Article IV Consultation, First Review Under the Extended Financing Facility and the Extended Credit Facility, and Request for Modification of Performance Criteria}

\author{
Informational Annex \\ Prepared by the Middle East and Central Asia Department
}

November 12, 2010

Contents

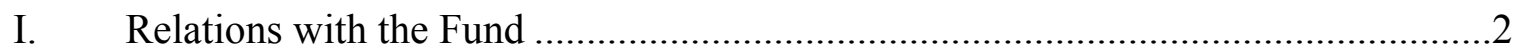

II. World Bank and IMF Collaboration - JMAP Implementation................................6

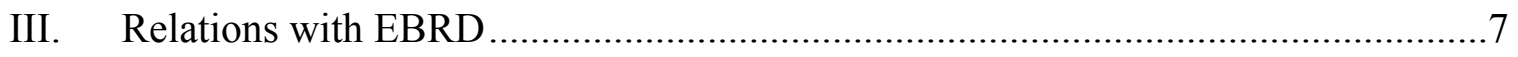

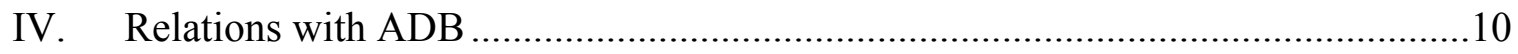

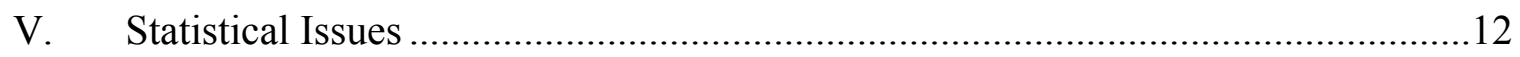




\section{ANNEX I. ARMENIA: RELATIONS WITH THE FUND}

(As of October 31, 2010)

I. Membership Status: Joined 05/28/1992; Article VIII

II. General Resources Account:

Quota

Fund holdings of currency

Reserve Tranche Position

III. SDR Department:

Net cumulative allocation

Holdings
SDR Million

92.00

459.93

0.00

SDR Million

87.99

24.78

SDR Million

350.43

81.11

17.50
Percent of Quota

100.00

499.92

0.00

Percent of Allocation

100.00

28.17

Percent of Quota

380.90

88.16

19.02

Extended Arrangements

\section{Latest Financial Arrangements:}

\begin{tabular}{|c|c|c|c|c|}
\hline Type & $\frac{\text { Approval }}{\underline{\text { Date }}}$ & $\frac{\text { Expiration }}{\text { Date }}$ & $\begin{array}{c}\text { Amount Approved } \\
\text { (SDR Million) }\end{array}$ & $\begin{array}{l}\text { Amount Drawn } \\
\text { (SDR Million) }\end{array}$ \\
\hline Stand-By & 03/6/09 & $06 / 27 / 10$ & 533.60 & 350.43 \\
\hline $\mathrm{ECF}$ & $06 / 28 / 2010$ & $06 / 27 / 2013$ & 133.40 & 18.70 \\
\hline $\mathrm{EFF}$ & $06 / 28 / 2010$ & $06 / 27 / 2013$ & 133.40 & 17.50 \\
\hline
\end{tabular}

\section{Projected Payments to Fund ${ }^{1 /}$}

(SDR Million; based on existing use of resources and present holdings of SDRs)

\begin{tabular}{|c|c|c|c|c|c|}
\hline & \multicolumn{5}{|c|}{ Forthcoming } \\
\hline & 2010 & 2011 & 2012 & 2013 & 2014 \\
\hline Principal & 4.23 & 14.44 & 101.00 & 180.22 & 98.08 \\
\hline Charges/interest & $\underline{1.75}$ & $\underline{7.28}$ & $\underline{7.01}$ & $\underline{3.88}$ & $\underline{1.45}$ \\
\hline Total & 5.98 & 21.72 & 108.01 & 184.10 & 99.53 \\
\hline
\end{tabular}

${ }^{1 /}$ When a member has overdue financial obligations outstanding for more than three months, the amount of such arrears will be shown in this section. 


\section{Safeguards Assessment}

Under the Fund's Safeguards Assessments policy, an update safeguards assessment of the CBA was completed in November 2010 with respect to the current EFF/ECF arrangements. The update assessment found that the CBA maintains a sound safeguards framework. The CBA's governance structure remains to be modernized to allow for more independent oversight, and a longer-term appointment of the external auditors would also improve audit efficiency and effectiveness.

\section{Exchange Rate Arrangement}

(a) The de jure arrangement is "free floating." The de facto arrangement was reclassified to "floating" from a "stabilized arrangement" effective March 3, 2009. The official exchange rate is quoted daily as a weighted average of the buying and selling rates in the foreign exchange market.

(b) Armenia maintains no exchange restrictions on the making of payments and transfers for current international transactions except for exchange restrictions maintained for security reasons, and notified to the Fund pursuant to Executive Board Decision No. 144-(52/51).

\section{Article IV Consultations}

The 2008 Article IV consultation with Armenia was concluded on November 17, 2008. Armenia is subject to a 24-month consultation cycle.

\section{FSAP Participation and ROSCs}

A joint World Bank-International Monetary Fund mission assessed Armenia's financial sector as part of the Financial Sector Assessment Program (FSAP) update during February 16-March 4, 2005. The Financial Sector Stability Assessment (FSSA) report was discussed by the Executive Board on May 25, 2005.

\section{Resident Representatives}

Mr. Guillermo Tolosa, since January 2010.

\section{Technical Assistance}

The following table summarizes the Fund's technical assistance to Armenia since 2002. 
Armenia: Technical Assistance from the Fund, 2002-10

\begin{tabular}{|c|c|c|c|}
\hline Subject & Type of Mission & Timing & Counterpart \\
\hline \multicolumn{4}{|l|}{ Fiscal Affairs Department (FAD) } \\
\hline Tax policy and administration & Short-term & $\begin{array}{l}\text { September 17-October } \\
2,2003\end{array}$ & $\begin{array}{l}\text { MFE, State Tax Service } \\
\text { (STS), and Customs } \\
\text { Committee }\end{array}$ \\
\hline Tax policy & Short-term & April 22-May 6, 2004 & $\begin{array}{l}\text { MFE, STS, and Customs } \\
\text { Committee }\end{array}$ \\
\hline Tax administration & Short-term & July $13-27,2004$ & $\begin{array}{l}\text { MFE, STS, and Customs } \\
\text { Committee }\end{array}$ \\
\hline $\begin{array}{l}\text { Public expenditure management } \\
\text { advisor }\end{array}$ & & $\begin{array}{l}\text { November 7, 2003- } \\
\text { November 6, } 2004\end{array}$ & \\
\hline Tax administration & Short-term & October 5-18, 2006 & MFE, STS \\
\hline Public financial management reform & Short-term & $\begin{array}{l}\text { November } 27- \\
\text { December } 8,2006\end{array}$ & MFE \\
\hline Tax administration & Short-term & $\begin{array}{l}\text { October } 31-\text { November } \\
13,2007\end{array}$ & MFE, STS \\
\hline Tax administration & Short-term & January $5-22,2008$ & MFE, STS \\
\hline Tax administration & Short-term & April 1-12, 2008 & MFE, STS \\
\hline Tax administration & Short-term & June 11-17, 2008 & MFE, STS \\
\hline Tax administration & Short-term & February 2-27, 2009 & MoF, SRC \\
\hline Tax administration & Short-term & April 28 - May 22, 2009 & MoF, SRC \\
\hline Tax administration & Short-term & $\begin{array}{l}\text { September } 23 \text { - October } \\
6,2009\end{array}$ & MoF, SRC \\
\hline Tax administration & Resident advisor & $\begin{array}{l}\text { March 1-May 14, } \\
2010\end{array}$ & MoF, SRC \\
\hline Tax administration & Resident advisor & $\begin{array}{l}\text { August } 30-\text { November } \\
10,2010\end{array}$ & MoF, SRC \\
\hline Tax administration & Short-term & September 2010 & MoF, SRC \\
\hline \multicolumn{4}{|l|}{ Legal Department } \\
\hline Tax legislation & & $\begin{array}{l}\text { September 1, 2004- } \\
\text { December 31, } 2005\end{array}$ & \\
\hline Legislation Development \& FIU & Short-term & July 31-August 7, 2006 & \\
\hline Unified Tax Code & Short-term & June $16-23,2007$ & MFE \\
\hline Unified Tax Code & Short-term & April 13-18, 2009 & MoF, SRC \\
\hline $\begin{array}{l}\text { AML-CFT National Strategies and } \\
\text { Coordination }\end{array}$ & Short-term & April 19-26, 2010 & CBA \\
\hline \multicolumn{4}{|c|}{ Monetary and Capital Markets Department } \\
\hline Banking system issues & Short-term & April 15-19, 2002 & CBA \\
\hline
\end{tabular}




\begin{tabular}{|c|c|c|c|}
\hline Subject & Type of Mission & Timing & Counterpart \\
\hline $\begin{array}{l}\text { Banking system, deposit insurance, } \\
\text { foreign exchange market development, } \\
\text { and CBA monetary operations. }\end{array}$ & Short-term & June 24-July 4, 2002 & CBA \\
\hline $\begin{array}{l}\text { Unified financial supervision, } \\
\text { mortgage financing markets and } \\
\text { inflation targeting }\end{array}$ & Short-term & $\begin{array}{l}\text { January 26-February } 6 \text {, } \\
2004\end{array}$ & CBA \\
\hline $\begin{array}{l}\text { Financial sector assessment program } \\
\text { update }\end{array}$ & Short-term & $\begin{array}{l}\text { February 16- } \\
\text { March 4, 2005 }\end{array}$ & CBA \\
\hline $\begin{array}{l}\text { Recapitalization of the Central Bank of } \\
\text { Armenia }\end{array}$ & Short-term & December 8-14, 2005 & CBA \\
\hline Monetary policy and markets & & $\begin{array}{l}\text { December 4, 2005- } \\
\text { December 31, } 2006\end{array}$ & \\
\hline $\begin{array}{l}\text { Strengthening the implementation of } \\
\text { monetary policy }\end{array}$ & Short-term & March 8-21, 2006 & $\mathrm{CBA}$ \\
\hline $\begin{array}{l}\text { Monetary policy implementation and } \\
\text { money market development }\end{array}$ & Short-term & October 5-17, 2006 & CBA \\
\hline AML/CFT preventive measures & & $\begin{array}{l}\text { April 1, 2006-April 30, } \\
2007\end{array}$ & \\
\hline $\begin{array}{l}\text { Inflation Targeting, Foreign Exchange } \\
\text { Market Development and Responding } \\
\text { to Dedollarization }\end{array}$ & Short-term & $\begin{array}{l}\text { August } 29 \text {-September } \\
10,2007\end{array}$ & CBA \\
\hline Exchange rate and collateral & Short-term & February $23-27,2009$ & CBA \\
\hline $\begin{array}{l}\text { Contingency planning, crisis } \\
\text { preparedness }\end{array}$ & Short-term & October $11-22,2009$ & CBA \\
\hline $\begin{array}{l}\text { Contingency planning, crisis } \\
\text { preparedness }\end{array}$ & Short-term & $\begin{array}{l}\text { March 28-April 14, } \\
2010\end{array}$ & CBA \\
\hline \multicolumn{4}{|l|}{ Statistics Department } \\
\hline Data dissemination standards & Short-term & September 18-25, 2003 & $\begin{array}{l}\text { National Statistical } \\
\text { Service }\end{array}$ \\
\hline Balance of payments: remittances & Short-term & $\begin{array}{l}\text { August 22-September } \\
5,2006\end{array}$ & $\mathrm{CBA}$ \\
\hline $\begin{array}{l}\text { Multitopic bundled mission: national } \\
\text { accounts, balance of payments, } \\
\text { monetary and financial statistics }\end{array}$ & Short-term & $\begin{array}{l}\text { January } 25 \text {-February } \\
5,2010\end{array}$ & $\begin{array}{l}\text { National Statistical } \\
\text { Service, CBA }\end{array}$ \\
\hline National accounts & Short-term & September 16-28, 2010 & $\begin{array}{l}\text { National Statistical } \\
\text { Service }\end{array}$ \\
\hline BOP statistics & Short-term & May 2011 & $\begin{array}{l}\text { National Statistical } \\
\text { Service, CBA }\end{array}$ \\
\hline \multicolumn{4}{|l|}{ International Capital Market Department } \\
\hline Sovereign credit quality & Short-term & September 6-10, 2005 & CBA \\
\hline Sovereign credit risk & Short-term & $\begin{array}{l}\text { August 20, 2005- } \\
\text { August 20, } 2006\end{array}$ & $\mathrm{CBA}$ \\
\hline
\end{tabular}




\section{ANNEX II. ARMENIA: WORLD BANK AND IMF COLLABORATION-JMAP IMPLEMENTATION (As of end-September, 2010)}

\begin{tabular}{|c|c|c|c|}
\hline Title & Products & $\begin{array}{l}\text { Provisional timing of } \\
\text { missions }\end{array}$ & $\begin{array}{l}\text { Expected delivery date } \\
\text { (tentative) }\end{array}$ \\
\hline \multicolumn{4}{|c|}{$\begin{array}{l}\text { 1. Bank work program } \\
\text { in next } 12 \text { months }\end{array}$} \\
\hline \multicolumn{4}{|c|}{ Selected Ongoing and New Operations } \\
\hline & Public Sector Modernization Project II (US\$9m) & Ongoing & $\begin{array}{l}\text { Project implementation started } \\
\text { in September } 2010\end{array}$ \\
\hline & $\begin{array}{l}\text { DPO-2 (Triggers on revenue administration, safety nets, business } \\
\text { environment, and mining code) (US } \$ 25 \mathrm{~m})\end{array}$ & Quarterly & December 2010 \\
\hline & E-Society \& Innovation Project (US\$24m) & Ongoing & August 2010 \\
\hline & North South Improvement Project 1 (US $\$ 43 m$ ) & Ongoing & July 2010 \\
\hline & $\begin{array}{l}\text { Community Agricultural Resource Management and Competitiveness } \\
\text { Project (US } \$ 14 \mathrm{~m} \text { ) }\end{array}$ & Ongoing & October 2011 \\
\hline & Electricity Supply Reliability and Energy Efficiency Project (US $\$ 36 \mathrm{~m})$ & Ongoing & November 2012 \\
\hline \multicolumn{4}{|c|}{ Analytical Work } \\
\hline & $\begin{array}{l}\text { Programmatic fiscal work (fiscal sustainability, revenue performance, } \\
\text { and expenditure rationalization) }\end{array}$ & $\begin{array}{l}\text { Quarterly missions, first } \\
\text { FY11 mission in } \\
\text { September } 2010\end{array}$ & $\begin{array}{l}\text { Draft policy notes in late 2010; } \\
\text { TA on DSA delivered by June } \\
2010\end{array}$ \\
\hline & Poverty monitoring and assessment & Continuous & $\begin{array}{l}\text { Annual series; } 2010 \text { report } \\
\text { delivered in June }\end{array}$ \\
\hline & $\begin{array}{l}\text { Trade analytical and TA work (on FTA. and export } \\
\text { diversification/expansion) }\end{array}$ & Early 2010 & End-December 2010 \\
\hline & Institutional and Governance Review & Mid-2010 & 2011 \\
\hline \multicolumn{4}{|c|}{ Selected Technical Assistance } \\
\hline & Public Debt Management IDF & Ongoing & June 2011 \\
\hline & Tax Audits and Macroprojections IDF & Starting in late 2010 & 2011 \\
\hline \multicolumn{4}{|c|}{$\begin{array}{l}\text { 2. IMF work program } \\
\text { in next } 12 \text { months }\end{array}$} \\
\hline & 2010 Article IV Consultation and First EFF/ECF Review & September 2010 & November 2010 \\
\hline \multicolumn{4}{|c|}{ Analyticial work on: } \\
\hline & $\begin{array}{l}\text { Analysis of the transmission mechanisms of monetary policy in } \\
\text { Armenia }\end{array}$ & May 2010 & December 2010 \\
\hline & Analysis of the equilibrium exchange rate & September 2010 & November 2010 \\
\hline \multicolumn{4}{|c|}{ Technical Assistance } \\
\hline & $\begin{array}{l}\text { MCM (Improvements to the inflation targeting regime; debt } \\
\text { management) }\end{array}$ & Early 2011 & Spring 2011 \\
\hline & $\begin{array}{l}\text { FAD (Tax Administration-Various operational issues to improve } \\
\text { revenue collection) }\end{array}$ & $\begin{array}{l}4 \text { Visits starting March } 1 \text {, } \\
2010 \text { through September } \\
2011 \text { (resident advisor) }\end{array}$ & September 2011 \\
\hline & $\begin{array}{l}\text { FAD (Tax Administration-Various operational issues to improve } \\
\text { revenue collection) }\end{array}$ & September 2010 & October 2010 \\
\hline & $\begin{array}{l}\text { FAD (Expenditure Policy-Operational issues to rationalize } \\
\text { expenditures) }\end{array}$ & Early 2011 & Spring 2011 \\
\hline & FAD (Tax Policy-Review of the draft tax code) & Early 2011 & Spring 2011 \\
\hline & STA (follow up mission on GDP statistics) & September 2010 & October 2010 \\
\hline & $\begin{array}{l}\text { STA (follow up mission on BOP statistics and moving BOP compilation } \\
\text { under the central bank }\end{array}$ & May 2011 & June 2011 \\
\hline \multicolumn{4}{|c|}{ 3. Joint work program } \\
\hline & Fiscal sustainability analysis inputs, including debt sustainability analysis & September 2010 & October 2010 \\
\hline & JSAN for SDS Update & Early 2011 & Early 2011 \\
\hline & FSAP Update & Mid-2011 & Late 2011 \\
\hline
\end{tabular}




\section{ANNEX III. ARMENIA: RELATIONS WITH THE EUROPEAN BANK FOR RECONSTRUCTION AND DEVELOPMENT (EBRD)}

(As of October 10, 2010)

1. As of October 10, 2010, the EBRD had approved 88 projects in Armenia in the power, transport, agribusiness, municipal and infrastructure, manufacturing and services, property, telecommunications, and financial sectors. Total commitments amounted to EUR 409 million.

2. There are four sovereign projects. First, the EBRD approved a sovereign-guaranteed loan of EUR 54.8 million for construction of the Hrazdan Unit 5 thermal power plant in March 1993, partly aimed at the eventual closure of Armenia's nuclear plant in Medzamor. The government was contemplating the privatization of Hrazdan Unit 5 as the completion of this plant was constrained by limited budgetary resources. The EBRD had funded technical assistance for the Hrazdan privatization prospectus and followed the privatization process. The Hrazdan Thermal Power Complex was transferred to the Russian Federation in the context of the debt-for-equity deal. Second, in November 1994, an agreement on a EUR 21.8 million loan to build an air cargo terminal in Zvartnots airport was signed under a guarantee by the Armenian government. The airport was transferred to private management in 2002 under a concession agreement. The new management has prepared a master plan for the development of the airport, which is expected to generate further cargo traffic. In April 2007, the EBRD approved a EUR 7 million loan to the State Committee for Water Systems, owner of the water and wastewater assets located in the small municipalities outside of Yerevan. The loan has been used to improve wastewater treatment in five municipalities located near Lake Sevan. In March 2010, EBRD signed a EUR 5 million sovereign loan with Yerevan Metro Company, covering immediate rehabilitation needs, safety upgrades, and energy efficiency.

3. Most of the EBRD's projects (96 percent) in Armenia are in the private sector. The Bank committed EUR 85.2 million to Armenia in 2009 through 11 transactions, all in the private sector. In addition to the loan to Electric Networks of Armenia (paragraph 7), the Bank approved an additional loan to Zvartnots International Airport of EUR 29.6 million (to be supplemented by investments from ADB and DEG). This project follows the successful completion in May 2007 of the first phase of the Passenger Terminal, for which the Bank provided a EUR 14.8 million loan together with DEG (USD 10 million). This project involves the construction and purchase of equipment for the second phase of the Passenger Terminal, with relocation of all arrivals and departures. The loan was a commercial facility with no sovereign support. Other private sector finance includes smaller loans and equity participation in a number of private companies. During January-October 2010, EBRD approved 17 projects with total commitment of EUR 36.7 million. The portfolio for 2010 comprises 10 projects in the financial institutions sector, 4 in agribusiness, one in each of the manufacturing and property sectors, and one sovereign project with Yerevan Metro Company. Two of the loans to financial institutions are in local currency. 
4. In the banking sector, equity participation in the Commercial Bank of GreeceArmenia (EUR 1.1 million) was approved in late 1999. Now there are four local banks where the EBRD participates in equity: Armeconombank, Byblos Bank Armenia, Ararat Bank, and Procredit Bank. The Bank also acquired an equity stake in an Armenian nonbank financial intermediary - Cascade Insurance and Reinsurance Company, an insurance subsidiary of Cascade Capital Holding.

5. The Armenia Multi-Bank Framework Facility II (AMBFF II), established to provide loans and equity to commercial banks and leasing companies, was approved in March 2006, in an amount of USD 40 million. The facility was extended by a further USD 80 million in November 2007. In late 2009, the EBRD approved a further USD 100 million extension to AMBFF II, to support increased financial intermediation and the development of the financial sector and to contribute to economic development by providing medium- to longterm funding to Armenian financial intermediaries. The Facility seeks to develop new products for financial institutions, including provision of local currency loans.

6. The EBRD has expanded its relationship with partner banks in Armenia from four to twelve (two EBRD partner banks-Ameria and Cascade will merge in 2010). Nine banks were provided with new credit facilities under the AMBFF. In 2010, EBRD has extended its portfolio under AMBFF by three more banks (Byblos, ArmSwiss, and Ameria ). One institution (Armeconombank) was provided with a mortgage facility, and the first leasing facility in Armenia was signed with ACBA Leasing in 2008 for EUR 5.9 million. Cofinancing with six local banks was extended, resulting in 14 sub-loans to Armenian corporates. By means of co-financing lines, the Bank has entered new sectors such as healthcare and telecommunications, in addition to significantly expanding its portfolio of agribusiness loans. A Trade Facilitation Program to facilitate access of Armenian banks to trade financing was also made available to nine banks.

7. Supporting development of renewable energy was another core activity of the Bank. The EBRD has joined forces with the World Bank, USAID, and Cascade Credit to launch the Armenian Renewable Energy Program (AREP). The Bank's participation took the form of a loan to Cascade Credit. The Bank also continued to finance renewable energy projects on its own through a Direct Lending Facility, with two projects signed. In addition to renewable energy, the Bank has sought to support post-privatization development of the energy sector with a loan to the privately-owned power distribution company. In April 2009, the EBRD signed a EUR 42 million loan with Electric Networks of Armenia to upgrade and modernize infrastructure and improve efficiency.

8. The EBRD launched Turn Around Management (TAM) and Business Advisory Service programs in Armenia in 2003, originally funded by the EU-Tacis program and now funded from the ETC Fund, to support micro, small, and medium-sized enterprises. Since 2003, BAS has completed 726 projects in an amount of EUR 2.7 million, while TAM delivered more than 20 projects. 
9. Projects identified by the Bank for future development are well diversified across sectors and include several relatively large transactions. New business opportunities are offered by the country's infrastructure sector, including in public transportation, water, sanitation, and solid waste treatment. The Bank is about to launch a USD 25 million program to finance projects for industrial energy efficiency and renewable energy through local banks.

10. As part of inspection reform and doing business programs, the EBRD is assisting the Government of Armenia to improve the business environment. The EBRD is promoting and supporting preparation of a corporate governance code along with the Ministry of Economy, the Central Bank, the Stock Exchange, and the IFC. The Bank is also providing assistance to the Public Services Regulatory Commission for telecommunications sector regulation.

11. The EBRD's current country strategy was approved in May 2009. Key priorities for the coming years are: (i) the financial sector; (ii) the enterprise sector, particularly SME and micro-enterprises financing through credit lines to Armenian banks or direct loans and equity investments; (iii) infrastructure investments in alternative energy sources and municipal projects; and (iv) policy dialogue with the government, other multilateral and bilateral donors, and other stakeholders. 


\section{AnNex IV. ARMEnia: Relations With the ASIAN DEVELOPMENT BANK (ADB)}

(As of mid-October 2010)

1. As of mid-October 2010, ADB had approved 5 projects in the transport, municipal infrastructure, and general budget support sectors. Total commitments amounted to USD 224 million.

2. All approved projects are sovereign. The first project is a Rural Roads Rehabilitation Sector Loan (USD 30.6 million) for rehabilitation of $220 \mathrm{~km}$ of rural roads approved in November 2007. The second is a Water Supply and Sanitation Sector Loan (USD 36 million) for repair and replacement of the water supply infrastructure in small towns and villages approved in December 2007. The third project is a Supplementary Loan (USD 17.3 million) to the Rural Roads Rehabilitation Project approved in November 2008, to finance an increase in project cost from higher construction materials prices, domestic inflation, and appreciation of the dram. The fourth is the Crisis Recovery Support Program Loan (USD 80 million) approved in July 2009, to protect budgetary social expenditures. And last is the first tranche (USD 60 million) of the North-South Road Corridor Investment Project approved in October 2009 for rehabilitation of $18.4 \mathrm{~km}$ of the four-lane Yerevan-Ashtarak highway and safety enhancement of a four-lane road between Yerevan and Ararat. All approved loans are from the ADB concessional window under the Asian Development Fund (ADF). ADB has not provided loans to Armenia from its nonconcessional window under the Ordinary Capital Resources (OCR).

3. In addition to loan projects, the ADB is also involved in non-lending operations, mostly Advisory and Capacity Development Technical Assistance. This is mostly researchoriented: a Transport Sector Development Strategy for 2009-19 (transport sector road map for 10 years); and Institutional Modernization to Improve the Business Environment (assisting the government with the introduction of online business registry system). The advisory technical assistance may lead to loan projects. The first tranche of the Armenian Urban Infrastructure Sustainable Development Program is an example where the project concept came from the Armenia Transport Strategy. The program will support the Yerevan west bypass road and provision of better access to the Yerevan metro.

4. To increase efficiency and long-term cooperation, the ADB has offered a new instrument: project funding under the Multi Finance Facility (MFF). The MFF allows approval of long-term sector assistance to be disbursed in tranches. Under the MFF, the ADB has approved USD 500 million for the North-South Road Corridor Investment Program. The funds will be available for 7 years, and the country would request them in tranches, with the first USD 60 million tranche already approved. The Armenia Urban Infrastructure Sustainable Development program is under preparation as an MFF-type project, with an indicative amount of up to USD 500 million. 
5. The ADB's economic report and the interim operational strategy for 2006-09 for Armenia were approved in 2006. The Country Operational Business Plan for 2008-10 was approved in 2007. In third quarter of 2011, the Bank is planning to prepare the Armenia Country Partnership Strategy for 5 years. The key priorities for the coming years are: (i) transport infrastructure; (ii) urban development; (iii) regional development; (iv) private sector development, particularly banking, finance, renewable energy, and transport; and (v) policy dialogue with the government, other multilateral and bilateral donors, and other stakeholders on country development prospects and reforms. 


\section{Annex V. Armenia: Statistical Issues}

1. Data provision has shortcomings, but is broadly adequate for surveillance. Further

improvements in real, fiscal, and external sector statistics would be desirable to facilitate enhanced design and monitoring of economic policies. The overall quality, timeliness, and coverage of macroeconomic statistics have improved significantly over the past few years. The Fund has substantially facilitated this process through technical assistance from the Statistics Department, the Fiscal Affairs Department, and the Monetary and Capital Markets Department. In November 2003, Armenia subscribed to the Special Data Dissemination Standard (SDDS). An April 2008 data ROSC mission prepared a detailed evaluation of the quality of the macroeconomic statistics. A multi-topic statistics mission visited Yerevan in February 2010 to review progress with implementation of past recommendations and follow up on outstanding issues in national accounts, balance of payments, and monetary and financial statistics. A follow up STA mission in September 2010 provided further guidance, focusing on improving the accuracy of annual and quarterly GDP estimates.

\section{Real sector statistics}

2. The National Statistics Service (NSS) compiles and disseminates annual and quarterly national accounts. In addition, a cumulative monthly GDP by production approach is compiled and disseminated 20 days after the end of each month. The NSS also compiles and disseminates annually a full set of accounts (up to financial accounts) for the total economy and by institutional sectors. The NSS is developing a plan for implementing the System of National Accounts 2008 (2008 SNA).

3. The accuracy of the annual estimates is undermined by the lack of exhaustive source data for informal activities and of appropriate price and volume indicators, particularly for construction activities. Construction output volume measures are derived by deflating current values with a price index for output, which uses weights and base year prices from a survey in 1984. To improve volume measures of construction, the NSS should start compiling a new construction output price index based on more sound methodology. Until the new construction price index becomes available, the NSS should use other indicators for deriving construction aggregates at constant prices. The NSS should also implement new surveys to derive a proper benchmark for informal activities.

4. The NSS compiles discrete quarterly GDP estimates by production and by expenditure approaches at current and at constant prices. The production-side estimates at current prices are derived partially from cumulative source data (from business statistics surveys) and partially from discrete data sources. The NSS validates and reconciles data from different sources, but the underlying problems associated with de-cumulating the cumulative output data distort the quarterly pattern. The NSS compiles and disseminates two sets of quarterly GDP volume measures - one at the prices of corresponding quarter of the previous year and the other at average prices of 2005. The first set does not constitute a time series 
since it does not allow a comparison of different periods. A multitopic IMF statistics technical assistance mission in January 2010 suggested that the NSS discontinue compiling these data. The compilation procedures for the second set (volume measures at average prices of 2005) need to be improved. The January 2010 mission also suggested that the NSS compile only one set of quarterly GDP estimates - quarterly GDP at previous-year average prices - and derive time series through chain-linking. These estimates would be conceptually consistent with the annual data. They would also allow comparisons between different periods, which is essential for analysis of business cycle.

5. The CPI covers 11 large population centers and Yerevan. Since January 2006, the CPI has been computed using 2005 weights. Concepts and definitions used in the compilation of the CPI are broadly in line with international standards; source data and compilation techniques are generally adequate. The NSS compiles a ten-day and a monthly CPI. The ten-day index and the monthly index are disseminated jointly. The February 2009 ROSC mission recommended development of an approach to include household expenditure on owner-occupied dwellings in the CPI calculations.

\section{Government finance statistics}

6. The budget execution reporting system compiles government finance data on a cash basis, supplemented with monthly reports on arrears and quarterly reports on receivables and payables. Daily revenue and cash expenditure data for the central government are available with a lag of one to two days. The ministry of finance $(\mathrm{MoF})$ is undertaking a comprehensive reform of the treasury system, including the introduction of an internal auditing system in line ministries and their respective budgetary institutions. A treasury single account (TSA) was introduced in 1996, and all bank accounts held by budgetary institutions were closed, except for project implementation units that are required by donors to operate with commercial bank accounts. Starting in 2002, some budgetary institutions have been converted into "noncommercial organizations" (NCOs). These units have been taken out of the treasury system and have their own bank accounts, but since 2003 report data on cash flows and balances to the MoF. The February 2009 ROSC report recommended including NCOs in the government finance statistics data published on national websites. These exceptions notwithstanding, all government receipts and payments are processed through the TSA, although there are still shortcomings on the timeliness and quality of data on the operations of local governments. Classification of government transactions by function and economic category are generally in line with the Manual on Government Finance Statistics 1986 , and monthly data on central government operations are disseminated one month after the reporting period.

7. The budget presentation and the classification of items under the economic and functional classification of expenditures need to be made more transparent; for instance, the data have been subject to frequent reclassification, and wages for military personnel are reported in the category of "other" goods and services rather than as a wage item. The 
February 2009 ROSC report recommended using market value rather than face value for financial assets other than loans, and for nonfinancial assets. The reconciliation of central government with general government operations is done by the NSS in cooperation with the MoF.

8. Since 2008, government finance statistics meet the classification requirements of the Government Finance Statistics Manual 2001 (GFSM 2001) for central government. Plans for improvement of the MoF envisage further progress in the next two years in implementing the GFSM 2001 classification for local government in 2009 and in accrual recording for all units of general government in 2010.

\section{Monetary and financial statistics}

9. Monetary and financial statistics are provided on a timely basis. Data on the accounts of the Central Bank of Armenia (CBA) are provided daily with a one-day lag, while monthly data on the monetary survey are provided with a three-week lag (and preliminary weekly data with a one-week lag). The balance sheets of the CBA and of the deposit money banks follow IAS methodology. Monthly interest rate data are provided with an one-week lag.

10. Responding to an IMF STA request, the CBA has compiled and submitted a complete set of monetary data beginning from December 2001 using Standardized Report Forms (SRF). STA validated the resulting monetary aggregates, and the data have been published since the December 2006 issue of IFS Supplement and are used to update IFS. An Integrated Monetary Database (IMD) has also been established by STA to share the SRF data with the IMF's Middle East and Central Asia Department.

\section{External sector statistics}

11. In 2009, the Armenian authorities decided to transfer the responsibility for compiling the balance of payments, international investment position (IIP), and external debt statistics from the NSS to the CBA. The NSS and CBA agreed on a target date for transfer beginning with data for the first quarter of 2011. The February 2010 mission agreed with the authorities on an action plan aimed at ensuring a smooth institutional transfer of responsibility, as well as consistency and continuity in the production of the external sector statistics. A follow up mission is scheduled for early 2011.

12. The coverage of external sector data has improved in recent years. Trade statistics are provided on a timely basis, and trade data by origin, destination, and commodity are generally available within a month. Price data for exports and imports are less readily available. Quarterly balance of payments statistics are generally available with a three-month lag. However, on remittances, which account for a significant part of the inflows, there are considerable discrepancies among available source data. Remittance data obtained from surveys are considerably lower than data obtained through the money transfer system. The NSS and CBA are working on establishing a compilation program that would enable 
improved measurement of remittances. The absence of a comprehensive, continuously updated business register hampers the coverage of transactions and institutional units; in particular, the coverage of the financial account items for the private nonbank sector.

13. Quarterly data on international investment position are published by the NSS within one quarter after the reference period, and the annual data within two quarters; and are also provided for publication in IFS. 
Armenia: Table of Common Indicators Required for Surveillance (As of October 15, 2010)

\begin{tabular}{|c|c|c|c|c|c|}
\hline & $\begin{array}{l}\text { Date of latest } \\
\text { observation }\end{array}$ & Date received & $\begin{array}{l}\text { Frequency of } \\
\text { Data }^{7}\end{array}$ & $\begin{array}{l}\text { Frequency of } \\
\text { Reporting }^{7}\end{array}$ & $\begin{array}{l}\text { Frequency } \\
\text { of publication }\end{array}$ \\
\hline Exchange Rates & Oct 2010 & $10 / 14 / 2010$ & $\mathrm{D}$ & $\mathrm{D}$ & D \\
\hline $\begin{array}{l}\text { International Reserve Assets and Reserve Liabilities of the Monetary } \\
\text { Authorities }^{1}\end{array}$ & Oct 2010 & $10 / 14 / 2010$ & $\mathrm{D}$ & D & M \\
\hline Reserve/Base Money & Oct 2010 & $10 / 14 / 2010$ & $\mathrm{D}$ & D & D \\
\hline Broad Money & Aug 2010 & $10 / 1 / 2010$ & M & M & M \\
\hline Central Bank Balance Sheet & Sep 2010 & $10 / 14 / 2010$ & $\mathrm{D}$ & M & M \\
\hline Consolidated Balance Sheet of the Banking System & Aug 2010 & $10 / 4 / 2010$ & M & M & M \\
\hline Interest Rates $^{2}$ & Sep 2010 & $10 / 4 / 2010$ & W & W & M \\
\hline Consumer Price Index & Sep 2010 & 9/30/2010 & M & M & M \\
\hline $\begin{array}{l}\text { Revenue, Expenditure, Balance and Composition of Financing }{ }^{3}-\text { General } \\
\text { Government }{ }^{4}\end{array}$ & Q2 2010 & $10 / 12 / 2010$ & Q & Q & Q \\
\hline $\begin{array}{l}\text { Revenue, Expenditure, Balance and Composition of Financing }{ }^{3} \text {-Central } \\
\text { Government }\end{array}$ & Aug 2010 & 9/30/2010 & M & M & Q \\
\hline Stocks of Central Government and Central Government-Guaranteed Debt ${ }^{5}$ & Aug 2010 & 9/30/2010 & M & M & Q \\
\hline External Current Account Balance & Q1 2010 & $9 / 10 / 2010$ & Q & Q & Q \\
\hline Exports and Imports of Goods and Services & Aug 2010 & $9 / 22 / 2010$ & M & M & Q \\
\hline GDP/GNP & Q2 2010 & $9 / 30 / 2010$ & Q & Q & Q \\
\hline Gross External Debt & Q1 2010 & $9 / 10 / 2010$ & Q & Q & Q \\
\hline International Investment Position ${ }^{6}$ & Q1 2010 & 9/10/2010 & $Q$ & $Q$ & Q \\
\hline
\end{tabular}

${ }^{1}$ Includes reserve assets pledged or otherwise encumbered as well as net derivative positions.

${ }^{2}$ Both market-based and officially determined, including discount rates, money market rates, rates on treasury bills, notes and bonds.

${ }^{3}$ Foreign, domestic bank, and domestic nonbank financing.

${ }^{4}$ The general government consists of the central government (budgetary funds, extrabudgetary funds, and social security funds) and state and local governments.

${ }^{5}$ Including currency and maturity composition.

${ }^{6}$ Includes external gross financial asset and liability positions vis-à-vis nonresidents.

${ }^{7}$ Daily (D), Weekly (W), Monthly (M), Quarterly (Q), Annually (A); Irregular (I); and Not Available (NA). 


\section{INTERNATIONAL MONETARY FUND}

EXTERNAL

RELATIONS

Public Information Notice

DEPARTMENT

Public Information Notice (PIN) No. 10/151

FOR IMMEDIATE RELEASE

December 1, 2010
International Monetary Fund

$70019^{\text {th }}$ Street, NW

Washington, D. C. 20431 USA

\section{IMF Executive Board Concludes 2010 Article IV Consultation for the Republic of Armenia}

On December 1, 2010, the Executive Board of the International Monetary Fund (IMF) concluded the 2010 Article IV consultation ${ }^{1}$ with the Republic of Armenia on a lapse of time basis. $^{2}$

\section{Background}

The global financial crisis brought to an end a long period of high, but unsustainable growth. Large remittances were funneled to construction and contributed to double-digit growth during 2000-07. These contracted during the crisis, along with exports and Foreign Direct Investment (FDI), so that the external current account deficit increased sharply and output declined abruptly, by over 14 percent in 2009 . A postponement of exchange rate adjustment led to a crisis of confidence, and a sharp fall in fiscal revenues added to concerns. The authorities' strong policy response, supported by the international community, helped alleviate the impact of the crisis and restore confidence, albeit at the cost of a substantial increase of public debt.

\footnotetext{
${ }^{1}$ Under Article IV of the IMF's Articles of Agreement, the IMF holds bilateral discussions with members, usually every year. A staff team visits the country, collects economic and financial information, and discusses with officials the country's economic developments and policies. On return to headquarters, the staff prepares a report, which forms the basis for discussion by the Executive Board. At the conclusion of the discussion, the Managing Director, as Chairman of the Board, summarizes the views of Executive Directors, and this summary is transmitted to the country's authorities. An explanation of any qualifiers used in summings up can be found here: http://www.imf.org/external/np/sec/misc/qualifiers.htm.
}

${ }^{2}$ This year's Article IV consultation was concluded on a lapse of time basis. Under the Fund's lapse of time procedures, the Executive Board completes Article IV consultations without convening formal discussions. 
Recovery is underway in Armenia in 2010, but the pace is slow. As the external environment has improved, industry and services are rebounding, but agriculture has been hit hard by adverse weather. With a rebound of agriculture, output is expected to grow by 4 percent in 2010 , slower than the $6 \frac{1}{2}$ percent pace registered in the first half of the year, and by $4 \frac{1}{2}$ percent next year. The contraction of agricultural output and a rise in imported wheat prices have translated into higher food prices. Comprising nearly half of the weight of the consumer price index, higher food prices have pushed annual inflation over 9 percent in recent months. However, as food price shocks recede, inflation should decline to 7 percent by end-2010, and with appropriate monetary and fiscal policies in place, to the central bank's target of $4 \pm 1.5$ percent during the second half of 2011 .

As the economy recovers, the authorities' focus is shifting to medium-term challenges, including restoring strong growth-from new sources-and reducing poverty, ensuring fiscal and debt sustainability, and maintaining financial sector stability. Post-crisis policies include fiscal consolidation, increased exchange rate flexibility, continued commitment to price stability, strengthening the banking sector, and stepping up structural reforms.

Fiscal consolidation has proceeded in 2010 . The crisis took a toll on public finances, with the overall deficit rising to 8 percent of Gross Domestic Product (GDP) last year. Fiscal performance has improved markedly in 2010, with tax collection increasing by 20 percent in the first eight months of the year, reflecting the rebound of activity, spending restraint, and progress in tax administration reforms. The fiscal adjustment is frontloaded, with the deficit targeted to come down by over 3 percentage points in 2010 and by a further 1 percentage point in 2011 and 2012. This will reduce public debt, which should peak at just below 50 percent of GDP in 2011, compared with 16 percent before the crisis. To protect social spending, the authorities are increasing the efficiency of health and education spending and improving the targeting and coverage of anti-poverty programs.

The authorities are committed to greater exchange rate flexibility, restricting interventions in the foreign exchange market to smoothing volatility and rebuilding reserves. Reflecting seasonal trends and external borrowing by domestic banks, the nominal exchange rate has appreciated by 10 percent since March. Limited foreign exchange purchases have helped rebuild reserves and stemmed a loss of competitiveness.

Monetary policy remains focused on price stability in an environment of higher dollarization, and efforts to strengthen the effectiveness of monetary policy continue. Liquidity conditions have eased with the central bank's foreign exchange purchases, and as the recent price pressures are mainly supply-driven, the central bank has kept the policy rate unchanged since May.

The banking sector remains stable, and credit has picked up in recent months, although mainly in foreign exchange. Prudential measures have focused on ensuring proper management of foreign exchange risks, by increasing capital and provisioning requirements. Nonperforming loans have declined from a peak of more than 10 percent last year to less than 5 percent at present. 
The authorities are pursuing structural reforms to improve competitiveness and raise Armenia's growth potential. These include strengthening the competition framework, further reducing barriers to entry for new businesses, and improving governance by addressing conflicts of interest of public officials. Measures to improve tax administration are also part of efforts to strengthen the business environment.

\section{Executive Board Assessment}

In concluding the 2010 Article IV consultation for the Republic of Armenia, Executive Directors endorsed the staff appraisal, as follows:

The Armenian economy suffered a major setback during the global crisis with output contracting by more than 14 percent in 2009. The swift policy response of the authorities, with the assistance of the international community, rightly shifted to a countercyclical mode, dampening the impact of the crisis, especially on the most vulnerable segments of the population.

Armenia is on the right trajectory as it exits the crisis. Recent macroeconomic developments are encouraging, despite some-largely supply driven-inflationary pressures. Fiscal consolidation is moving forward, while the monetary policy stance remains neutral. Structural reforms have also progressed, but need to proceed faster to raise the growth potential of the economy.

Over the medium term, Armenia faces several challenges precipitated by the crisis. The external environment is difficult, and external imbalances must to be tackled expeditiously to avoid a disorderly adjustment. Decisive fiscal consolidation is needed to preserve debt sustainability and support medium-term external adjustment. Broader reforms are essential to promote growth and poverty reduction, namely, to address significant weaknesses in tax administration and the business environment, strengthen financial stability, and deepen financial intermediation.

The government's fiscal policy strategy appropriately addresses the medium-term fiscal and debt vulnerabilities. With the economy gradually recovering, the fiscal stimulus is being gradually withdrawn. The budget deficit is projected to decline by 3 percent of GDP in 2010 and by a further 1 percent of GDP in 2011. The focus of consolidation is appropriately on enhancing revenue collection, rather than expenditure compression that could hurt spending on important sectors-education and health-as well as the poor. With these policies, the fiscal deficit is projected to fall to about 2 percent of GDP in the medium term, which will ensure that public debt remains sustainable. The MTEF and the new debt management strategy should substantially contribute to sound public finances.

The CBA's monetary policy response since the crisis has been appropriate. The move to a more flexible exchange rate, the gradual lowering of the policy rate during the crisis, and its gradual increase as the recovery started were steps in the right direction. In the near term, 
monetary policy should continue to remain vigilant, increasing the policy rate if demand pressures or second-round effects from food price shocks arise. The CBA should continue to improve its communications to ensure that inflation expectations remain anchored around the inflation target. Planned dedollarization efforts could facilitate the monetary transmission mechanism, but sustained and sound macroeconomic policies are essential to reduce dollarization.

The increased flexibility of the exchange rate is serving Armenia well. The authorities' commitment to maintaining a flexible exchange rate is welcome. The CBA intervention policy should continue to aim at only smoothing sharp fluctuations in the market, while guarding foreign exchange reserves and ensuring that the economy remains competitive. Any appreciation bias in the interventions will further contribute to a misalignment of the exchange rate with the equilibrium rate and delay external adjustment.

Armenia's banking sector remains sound and proved resilient to the global shocks. After rising in the first half of 2009, NPLs came down and have remained at low levels throughout 2010. Staff is encouraged by the recent pick up in lending to the private sector. It is essential that the authorities continue to implement measures to preserve financial stability, including strengthening the supervision framework, tightening foreign exchange-related prudential regulations, and improving crisis preparedness and contingency planning.

Further improvements in tax administration will be needed to support fiscal consolidation and achieve stronger growth. While the government's commitment to reforming the tax administration is welcome, efforts to strengthen tax administration and advance tax policy reforms have had mixed success, in part reflecting political economy constraints. The recent actions are a step in the right direction, but decisive implementation in line with international best practices will be crucial to realize gains.

Structural reforms will be essential if Armenia is to enhance its competitiveness and raise its growth potential. Bolder and deeper reforms are needed to improve governance, enhance competition, diminish monopolistic behavior, diversify exports, and more generally, modernize the economy. Measures to address conflicts of interest of public officials, strengthen the competition framework, and reduce barriers to entry by firms are welcome and should be followed by further trade reforms.

Public Information Notices (PINs) form part of the IMF's efforts to promote transparency of the IMF's views and analysis of economic developments and policies. With the consent of the country (or countries) concerned, PINs are issued after Executive Board discussions of Article IV consultations with member countries, of its surveillance of developments at the regional level, of post-program monitoring, and of ex post assessments of member countries with longer-term program engagements. PINs are also issued after Executive Board discussions of general policy matters, unless otherwise decided by the Executive Board in a particular case. 
Armenia: Selected Economic Indicators

\begin{tabular}{|c|c|c|c|c|c|}
\hline & 2007 & 2008 & 2009 & $\begin{array}{l}2010 \\
\text { Proj. }\end{array}$ & $\begin{array}{l}2011 \\
\text { Proj. }\end{array}$ \\
\hline \multicolumn{6}{|l|}{ Real Sector } \\
\hline Real GDP growth (percentage change) & 13.7 & 6.9 & -14.2 & 4.0 & 4.6 \\
\hline GDP (in millions of U.S. dollars) & 9,206 & 11,662 & 8,541 & 8,836 & 8,858 \\
\hline GDP per capita (in U.S. dollars) & 2,853 & 3,606 & 2,615 & 2,678 & 2,658 \\
\hline \multicolumn{6}{|l|}{ Inflation (in percent) } \\
\hline Period average & 4.4 & 9.0 & 3.5 & 7.8 & 5.5 \\
\hline End-of-period & 6.6 & 5.2 & 6.6 & 7.1 & 4.6 \\
\hline \multicolumn{6}{|l|}{$\begin{array}{l}\text { Central government operations (in percent } \\
\text { of GDP) }\end{array}$} \\
\hline Revenue and grants & 20.1 & 20.5 & 21.1 & 21.8 & 22.7 \\
\hline Expenditure and net lending & 22.4 & 22.2 & 28.9 & 26.6 & 26.6 \\
\hline Overall balance (cash basis) & -2.2 & -1.2 & -8.0 & -4.8 & -3.9 \\
\hline \multicolumn{6}{|l|}{ Monetary indicators } \\
\hline $\begin{array}{l}\text { Reserve money (end-of-period growth rate, in } \\
\text { percent) }\end{array}$ & 50.9 & 5.3 & 13.8 & 5.5 & 10.4 \\
\hline $\begin{array}{l}\text { Broad money (end-of-period growth rate, in } \\
\text { percent) }\end{array}$ & 42.3 & 2.4 & 16.4 & 13.1 & 13.5 \\
\hline Broad money velocity & 4.6 & 5.0 & 3.8 & 3.7 & 3.6 \\
\hline \multicolumn{6}{|l|}{ External Sector } \\
\hline \multicolumn{6}{|l|}{ Current account balance (including transfers) } \\
\hline In millions of U.S. dollars & -589 & $-1,382$ & $-1,367$ & $-1,289$ & $-1,112$ \\
\hline In percent of GDP & -6.4 & -11.8 & -16.0 & -14.6 & -12.6 \\
\hline \multicolumn{6}{|l|}{ External debt } \\
\hline In millions of U.S. dollars & 1,449 & 1,577 & 2,967 & 3,336 & 3,700 \\
\hline $\begin{array}{l}\text { In percent of exports of goods and services } \\
\text { Gross official international reserves }\end{array}$ & 81.6 & 89.8 & 222.2 & 220.4 & 215.1 \\
\hline In millions of U.S. dollars & 1,659 & 1,407 & 2,004 & 1,740 & 1,790 \\
\hline In months of imports of goods and services & 4.2 & 4.6 & 6.2 & 5.1 & 4.9 \\
\hline
\end{tabular}

Sources: Armenian authorities; and IMF staff estimates. 
Press Release No. 10/465

International Monetary Fund

FOR IMMEDIATE RELEASE

Washington, D.C. 20431 USA

December 1, 2010

\section{IMF Executive Board Completes First Review Under EFF/ECF Arrangement for Armenia and Approves US\$57.6 Million Disbursement}

The Executive Board of the International Monetary Fund (IMF) has completed its first review of Armenia's economic performance under a program supported by the Extended Fund Facility (EFF) and the Extended Credit Facility arrangement (ECF). ${ }^{1}$ The Board's decision was taken on a lapse of time basis ${ }^{2}$.

The decision enables the authorities to draw an additional SDR 39.0 million (US\$57.6 million), bringing total disbursements under the arrangement to an amount equivalent to SDR 78.1 million (US\$115.2 million).

The three-year SDR 266.8 million (about US\$393.9 million) EFF and ECF arrangement with Armenia was approved by the IMF's Executive Board on June 28, 2010 (see Press Release No. $\underline{10 / 263})$.

\footnotetext{
${ }^{1}$ The Extended Credit Facility (ECF) has replaced the Poverty Reduction and Growth Facility (PRGF) as the Fund's main tool for medium-term financial support to low-income countries by providing a higher level of access to financing, more concessional terms, enhanced flexibility in program design features, and more focused streamlined conditionality. Financing under the ECF carries a zero interest rate, with a grace period of $51 / 2$ years, and a final maturity of 10 years (http://www.imf.org/external/np/exr/facts/ecf.htm). The Fund reviews the level of interest rates for all concessional facilities every two years.

${ }^{2}$ The Executive Board takes decisions under its lapse of time procedure when it is agreed by the Board that a proposal can be considered without convening formal discussions.
} 